\title{
Efficient Control Based on a Verified Model for an Autonomous Underwater Vehicle - A Case Study of the Autonomous Benthic Explorer
}

\author{
by \\ Jamie Marie Anderson \\ B.S. Mechanical Engineering \\ University of California, San Diego (1989) \\ Submitted to the Massachusetts Institute of Technology \\ and Woods Hole Oceanographic Institution \\ in partial fulfillment of the requirements for the degree of \\ Master of Science in Oceanographic Engineering \\ at the

\begin{tabular}{c|c} 
at the & $\begin{array}{c}\text { MARIRE } \\
\text { BOLOGICAL } \\
\text { LABORATORY }\end{array}$ \\
\hline and the & $\begin{array}{c}\text { WORSACHUS HOLE, MASS. } \\
\text { W. H. O. I. }\end{array}$ \\
\hline
\end{tabular}

\section{WOODS HOLE OCEANOGRAPHIC INSTITUTION}

February 1992

(C)Jamie Marie Anderson, MCMXCII

The author hereby grants to MIT and WHOI permission to reproduce and to distribute copies of this thesis document in whole or in partis

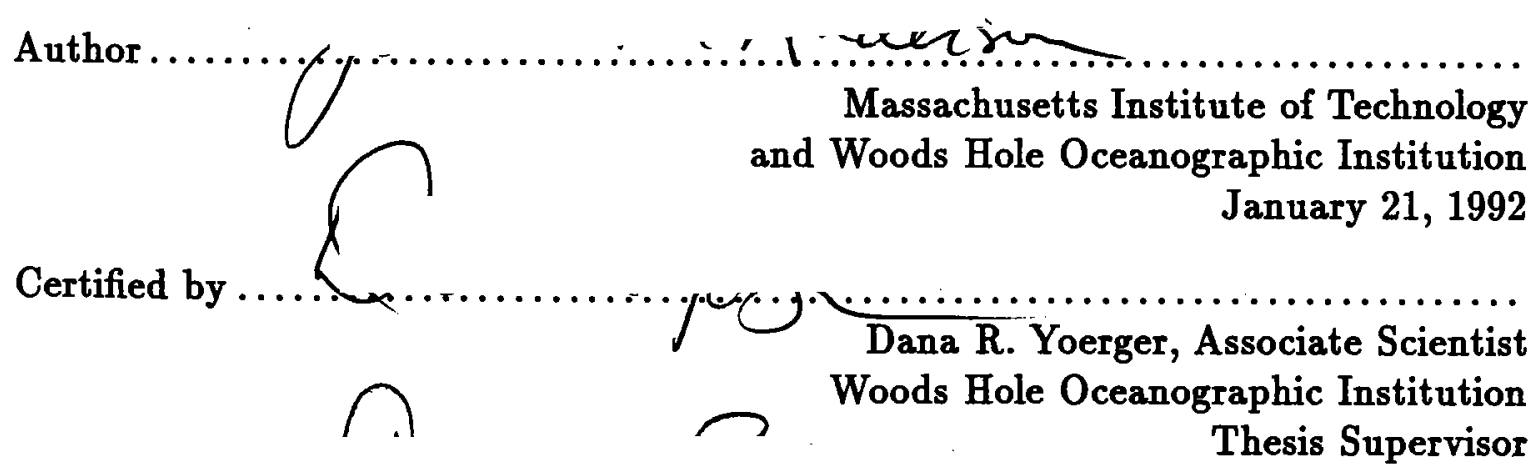

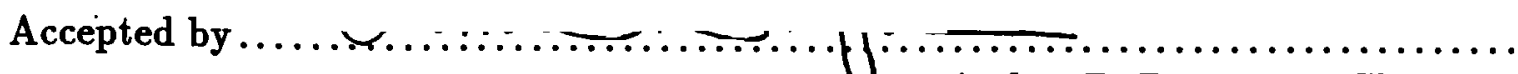




\title{
Efficient Control Based on a Verifled Model for an
}

\section{Autonomous Underwater Vehicle - A Case Study of the}

\author{
Autonomous Benthic Explorer
}

by

\author{
Jamie Marie Anderson \\ Submitted to the Massachusetts Institute of Technology \\ and Woods Hole Oceanographic Institution \\ on January 21, 1992, in partial fulfillment of the \\ requirements for the degree of \\ Master of Science in Oceanographic Engineering
}

\section{Abstract}

The Autonomous Benthic Explorer (ABE) is an unmanned underwater vehicle being developed for scientific study of the deep ocean seafloor. ABE will be completely autonomous from the surface which means that the lifetime of the mission will depend largely on how the vehicle is controlled. An accurate system model is critical for the controller development and trajectory planning.

A model of the ABE vehicle dynamics is formulated for surge, heave and pitch motions. These motions in the longitudinal plane are particularly important for the basic ABE trajectories of forward flight, depth changes and maneuvers involving both. A scale model of the ABE vehicle was towed to determine the lift/drag relationships to nonzero angles of attack. The experimental results are uaged in conjunction with traditional analytical techniques to generate a model of the longitudinal dynamics.

The ABE model was studied in simulation over anticipated vehicle trajectories. A proportional plus derivative controller and a sliding mode controller were developed for tracking control. The power consumptions for different controllers and trajectories are examined. The results of this study will be incorporated in the final ABE design.

Thesis Supervisor: Dana R. Yoerger

Title: Associate Scientist

Woods Hole Oceanographic Institution 


\section{Acknowledgments}

This work was make possible by the support of my friends and family. In particular, I would like to thank Dana Yoerger for his enthusiasm and help in making me a better detective. I want to specially thank Torsten Berger for this work could not have been completed without his love and patient support.

The Office of Naval Research is gratefully acknowledged for its financial support of my graduate education. In addition, this work has been sponsored in part by the National Science Foundation, grant number OCE 8820227.

$$
* 1+2
$$




\section{Contents}

1 Introduction $\quad 0$

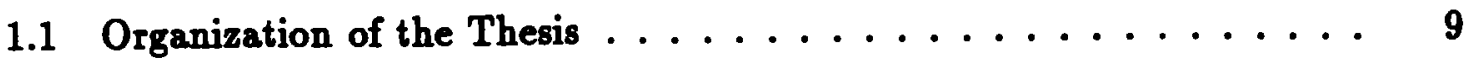

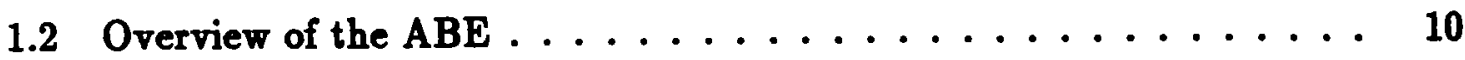

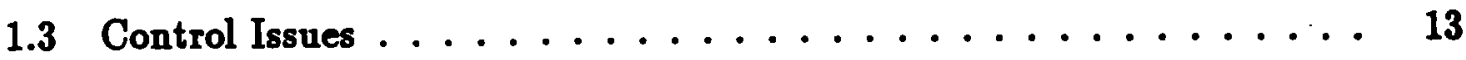

2 Scale Model Tests 15

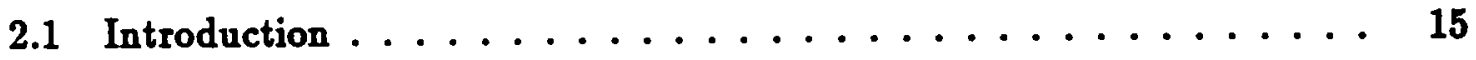

2.2 Experimental Setup .......................... 15

2.3 Experimental Procedure . . . . . . . . . . . . . 18

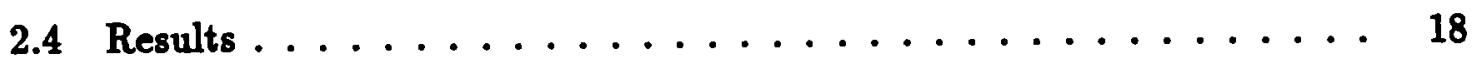

2.5 Comparison with Simple Drag Models . . . . . . . . . . 32

3 The ABE Model $\quad 37$

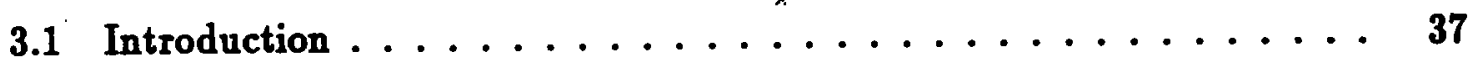

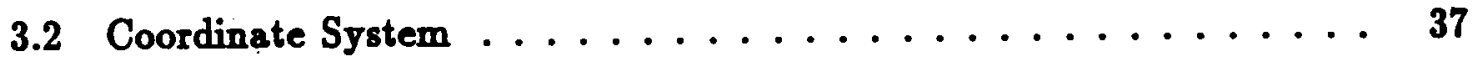

3.3 Formulation of the Equations of Motion ........... 38

3.4 Modelling the Forces on the Vehicle . . . . . . . . . . . 40

3.4.1 Gravity and Buoyancy Forces ............... 40

3.4.2 Fluid Inertial Forces ................... 40

3.4.3 Viscous Forces . . . . . . . . . . . . . . . 43

3.4.4 Thruster Model . . . . . . . . . . . . . . . . 46

3.4.5 Unmodelled Forces .................. . . 49

3.5 Summary of Equations of Motion . . . . . . . . . . . . 49 
4 Simulation of the ABE $\quad 51$

4.1 Introduction ....................... 51

4.2 Power States ........................ 51

4.3 Diagnostics ........................ 52

4.4 Problems with Simulation .................. 57

5 Controller Design $\quad 61$

5.1 Introduction ........................ 61

5.2 Proportional Plus Derivative (PD) Control . . . . . . . . . . . 62

5.3 Sliding Mode Control . . . . . . . . . . . . . . . . 63

6 Evaluation of Controller Performance $\quad 70$

6.1 Introduction $\ldots \ldots \ldots \ldots$

6.2 Trajectories of Interest ................... 70

6.3 Performance ....................... 71

6.4 Combined Forward/Vertical Motion Revisited ............ 88

7 Conclusions and Recommendations for Future Work 91

A Refining the Equations of Motion 93 


\section{List of Figures}

$1-1$ ABE Mission Summary $\ldots \ldots \ldots \ldots \ldots \ldots \ldots$

$1-2$ ABE Mechanical Layout $\ldots \ldots \ldots \ldots \ldots \ldots$

2-1 Scale Model of ABE vehicle $\ldots \ldots \ldots \ldots \ldots \ldots \ldots$

$2-2$ Experimental Setup . . . . . . . . . . . . 17

2-3 Typical Experimental Data $\ldots \ldots \ldots \ldots \ldots \ldots$

$2-4$ Forces and Moments on the Model . . . . . . . . . . 21

$2-5$ Square Law Drag Behavior . . . . . . . . . . . . . 22

2-6 Forces and Moments on the Pitched Model . . . . . . . . . 23

2-7 Measured Drag Forces as a Function of Pitch Angle . . . . . . . . 26

2-8 Drag Coefficient as a Function of Pitch Angle . . . . . . . 27

2-9 Lift Force as a Function of Pitch Angle . . . . . . . . . . 29

2-10 Lift Coefficient as a Function of Pitch Angle $\ldots, \ldots, \ldots \ldots$

2-11 Measured Lift Forces Compared to Data for Bodies of Revolution . . 31

$2-12$ Model A . . . . . . . . . . . . . . . 32

2-13 Performance of Model A . . . . . . . . . . . . . 33

$2-14$ Model B . . . . . . . . . . . . . . . . . 34

2-15 Model B-Drag Force $\ldots \ldots \ldots \ldots \ldots \ldots \ldots \ldots$

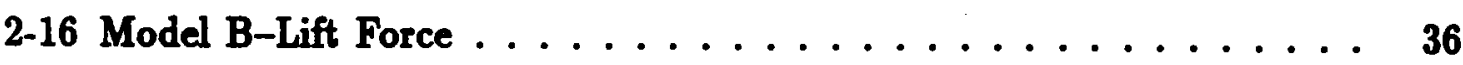

3-1 Body Coordinate System Definitions $\ldots \ldots \ldots \ldots \ldots \ldots$

3-2 Assumed Forces and Moments on the Vehicle . . . . . . . . . . 44

3-3 Strip Theory Formulation for Pitch Damping . . . . . . . . . 45

3-4 ABE Thruster Locations . . . . . . . . . . . . . . 47 
3-5 Thruster Step Responses . . . . . . . . . . . . . . . . 48

4 -1 Response to 10 Degree Initial Pitch Angle . . . . . . . . . 53

4-2 Response to Initial Conditions: $u(0)=.50 \mathrm{~m} / \mathrm{s}, w(0)=.25 \mathrm{~m} / \mathrm{s} \ldots 55$

4-3 Initial Condition Response with a Disturbance . . . . . . . . 56

4-4 Response to $10 \mathrm{~N}$ Step Command on Main Forward Thruster . . . . 58

4-5 Response to a $10 \mathrm{~N}$ Step Command on Aft Vertical Thruster . . . . 59

6-1 PD Control Performance on Track I . . . . . . . . . . . 73

6-2 PD Control Power Consumption on Track I . . . . . . . . . 74

6-3 Sliding Mode Control Performance on Track I . . . . . . . . 75

6-4 Sliding Mode Control Power Consumption on Track I . . . . . . . 76

6-5 Bandwidth $/$ Pest for Sliding Mode Control on Track I . . . . . . 77

6-6 PD Control Performance on Track II . . . . . . . . . . . . 79

6-7 PD Control Power Consumption on Track II . . . . . . . . . . 80

6-8 Sliding Mode Control Performance on Track II . . . . . . . . . . . . 81

6-9 Sliding Mode Control Power Consumption on Track II . . . . . . 82

6-10 PD Control Performance on Track III . . . . . . . . . . . . . . 84

6-11 PD Control Power Consumption on Track III . . . . . . . . 85

6-12 Sliding Mode Control Performance on Track III . . . . . . . . . . . 86

6-13 Sliding Mode Control Power Consumption on Track III . . . . . . 87

6-14 Forward/Vertical Maneuver with Pitch Angle Control . . . . . . . 89

6-15 Forward/Vertical Maneuver without Pitch Control . . . . . . . . 90 


\section{List of Tables}

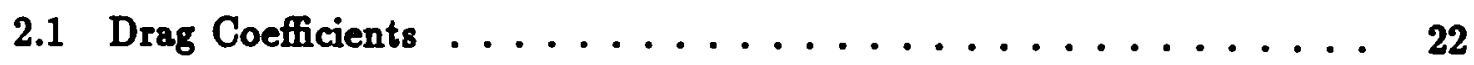

2.2 Sensitivity Study Maximum Errors $\ldots \ldots \ldots \ldots \ldots$

3.1 Summary of Vehicle Parameters . . . . . . . . . . . 50

5.1 Allowable Parameter Variations . . . . . . . . . . 65

6.1 Controller Performance Comparison . . . . . . . . . . . . 83

6.2 Average Power Requirement for Cruising . . . . . . . . . . . 85 


\section{Chapter 1}

\section{Introduction}

\subsection{Organization of the Thesis}

The Autonomous Benthic Explorer (ABE) is an underwater vehicle which is under development at the Woods Hole Oceanographic Institution. The vehicle is designed for deep ocean survey tasks in complete autonomy from the surface. The life span of the ABE mission will be dependent on a finite battery lifetime. Efficient maneuvers are therefore critical to the ABE mission. This thesis addresses the problem of controlling the vehicle in the most efficient possible manner to accomplish anticipated mission requirements. Since most ABE trajectories will involve forward motion and depth changes, this work addresses the dynamics in the longitudinal plane.

The thesis is organized as follows. First, a model of the vehicle dynamics is formulated for the longitudinal plane of motion. Chapter 2 describes a scale model experiment done to understand the basic drag/lift behavior of the ABE geometry. In chapter 3, the development of the complete dynamic model is presented. Established techniques are used to approximate the hydrodynamic forces on the vehicle for the longitudinal plane of motion. The experimental results are incorporated into the vehicle model.

In chapter 4 the simulation of the vehicle is discussed. Issues related to simulation are considered and some diagnostics are presented. Chapter 5 details the design of a sliding mode controller and a proportional plus derivative controller. In chapter 6 , 
the performances of the two controllers are compared over some typical ABE trajectories. The power consumption problem is addressed for combined forward/vertical maneuvers. Finally, chapter 7 presents the conclusions and recommendations for implementation and future work.

\subsection{Overview of the ABE}

The Autonomous Benthic Explorer, as its name implies, is an underwater vehicle designed for deep seafloor survey. The motivation for a vehicle of this type is that scientists interested in the deep ocean currently have no inexpensive, mobile instrument platforms for such environments. The ABE is designed such that it can remain at the site of interest for long periods of time and record data frequently. Typically, ABE would repeat a photo survey once a month for up to one year. ABE will complement the capabilities of moored instrument arrays because of its mobility. Scientists will be able to observe time and spatially varying phenomenon on the ocean floor that have never been documented before.

The ABE will have no ship support during its mission. Figure 1-1 shows a pictorial summary of the mission. When deployed from a ship, the ABE will descend to the bottom where there will be an acoustic beacon and a hitching station. An acoustic navigation network will already be in place at the site. Thy $\mathrm{ABE}$, will attach itself to the hitching station for periods of sleep for which nearly all systems will be turned off to conserve battery power.

The ABE will wake itself for self tests and for preprogrammed survey tasks. A typical survey would involve taking visual images over a bottom following trajectory. The ABE design is capable of including various sensors which can record video and measure temperature, conductivity, etc.

The ABE project is intended to bridge the gap between fixed instrument platforms and large scale remotely operated vehicles. The $\mathrm{ABE}$ will provide a mobile instrument package which does not require expensive ship or personnel support at the surface. A complete description of the ABE system is given in [16]. 


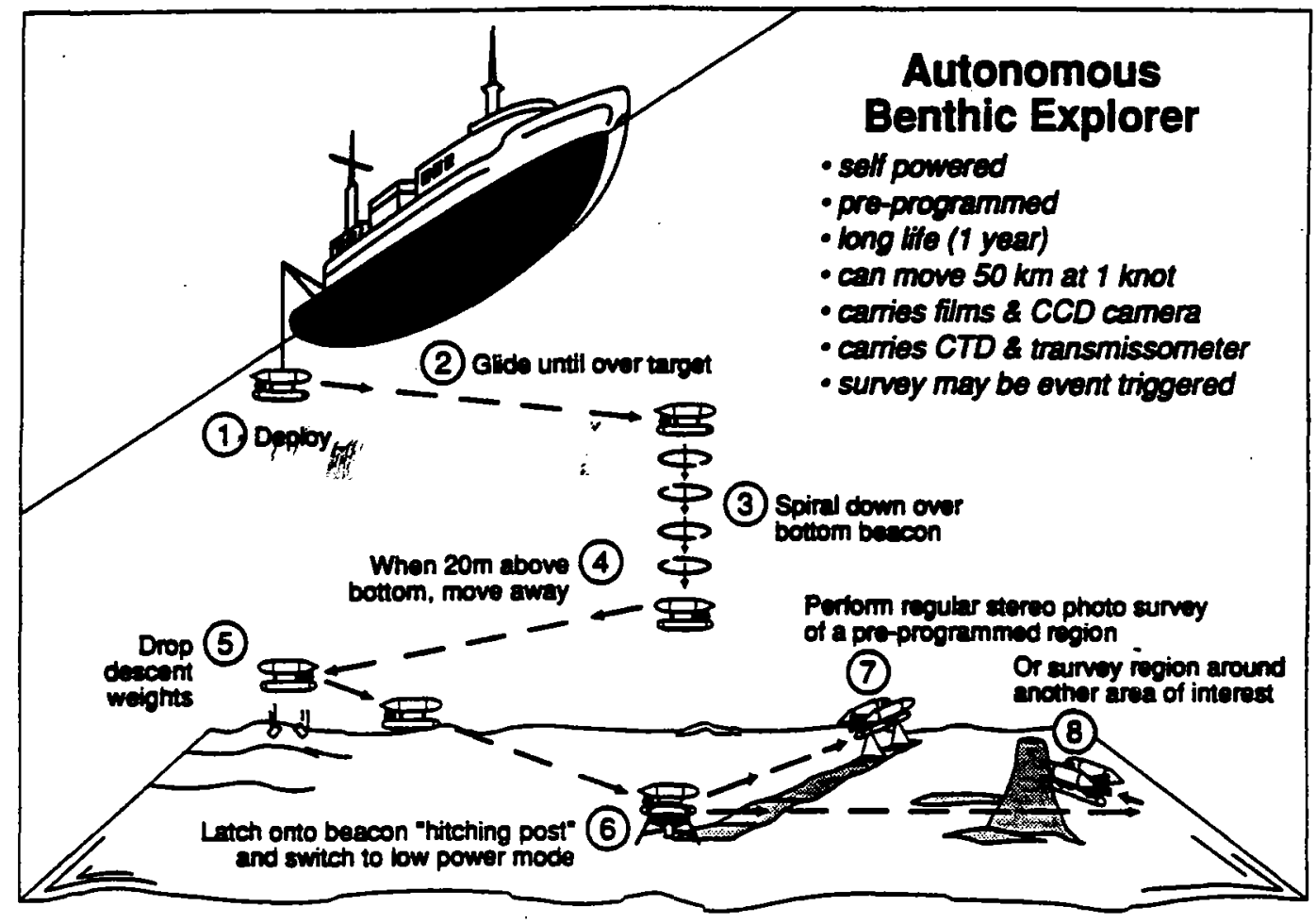

Figure 1-1: ABE Mission Summary 


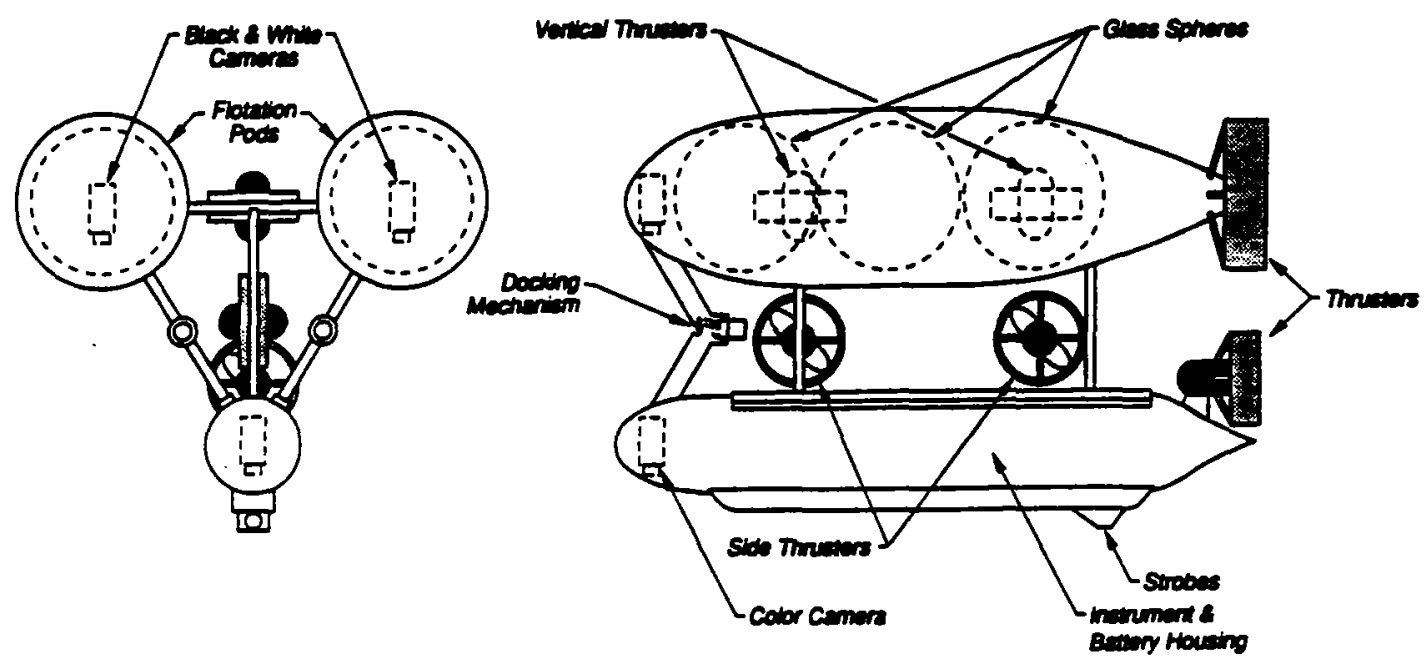

Figure 1-2: ABE Mechanical Layout

The mechanical layout of the ABE vehicle is shown in figure 1-2. The design is built around six 17 inch $(43 \mathrm{~cm})$ glass spheres which provide the required buoyancy. These spheres have been used for many years by the oceanographic community for subsurface floats and have shown great reliability. The six spheres are packed together into two buoyancy pods which are streamlined with a fiberglass hull.

The electronics are enclosed in an aluminum pressure vessel which lies below the buoyancy pods. This payload pod will carry all of the electronics and batteries for the vehicle. Extra sensors will also be attached to this pod.

The ABE is designed to operate at a cruise velocity of $1 \mathrm{knot}(.5 \mathrm{~m} / \mathrm{s})$ with the capability of a sprint velocity of 2 knots. The power budget allows 100 watts for normal propulsion and 200 watts peak power for sprint maneuvers.

The ABE will have active control in five degrees of freedom. The only passive response will be the roll motion of the vehicle. The forward direction will have three thrusters, one behind each pod. There will be two thrusters for the vertical direction 
and two in the lateral direction. In addition, there will be a mechanism for pitching the vehicle by moving a weight inside of the payload pressure vessel.

\subsection{Control Issues}

The ABE mission life is directly related to the manner in which the vehicle is controlled. There are four principal motions of interest:

1. Level flight over large distances. Thrust is divided among the three forward thrusters to move the vehicle forward.

2. Vertical flight over short distances. The two vertical thrusters are used to power the vehicle straight up or down" This motion would be used extensively in the hitching process.

3. Coordinated forward flight with depth change. Five thrusters are used in conjunction with the motion of an internal weight (for pitch control) to move the vehicle forward and up or down at the same time. This would be the primary mechanism for changing depth since the drag force for forward motion is much less than that for vertical motion.

4. Hover mode. All thrusters are used to keep the vehicle on station for hitching or for the collection of data.

All of these trajectories have one very important aspect in common. They are all concerned with the longitudinal plane, that is, surge, heave and pitch motions. Therefore, the primary objective is to formulate a good model of these dynamics.

The goal of this thesis is study the problem of controlling the ABE efficiently in the longitudinal plane. A model is developed which includes analytical estimates of the hydrodynamic force, experimentally measured drag forces and a verified model of the thrusters. This model is then examined in simulation and used to test controllers and control strategies. 
Two different controllers are developed and studied over various trajectories. The actual power requirements are calculated which is a great improvement on the standard procedure for budgeting power. Usually one would simply compute the steady state drag force and multiply it by the cruise velocity and the thruster efficiency. This approach gives large errors because it does not consider the control actions needed to accelerate the vehicle to cruise velocity and keep it on course. The actual power requirement under closed loop control is significantly higher and can only be predicted with a good simulation model. The results of this work will be implemented in the ABE design and operation. 


\section{Chapter 2}

\section{Scale Model Tests}

\subsection{Introduction :}

A one-third scale model of the ABE vehicle was towed at the MIT Ocean Engineering Towing Tank to determine some of its hydrodynamic properties. Specifically, the drag and lift forces were measured for different angles of attack and different towing velocities. The lift and drag behaviors observed with the scale model will be used explicitly in the simulation of the full scale vehicle model.

\subsection{Experimental Setup}

Figure 2-1 shows the scale model of the ABE vehicle. It consists of two wooden buoyancy pods which are 5.8 inches $(15 \mathrm{~cm})$ in diameter and 23.4 inches $(59 \mathrm{~cm})$ long. The nose caps are hemispherical and the tails are constructed such that the profile is a smooth transition to a sharp trailing edge. These pods are connected to a similar 4 inch $(10 \mathrm{~cm})$ diameter, 24 inch $(60 \mathrm{~cm})$ long $P V C$ tube which has a wooden nose and tail.

These three structures are joined together with aluminum struts which are streamlined in the forward direction. The three pods are in a triangular arrangement with an angle of 53 degrees between the diagonal struts and the horizontal. The angle between the struts on the lower pod is 74 degrees. 


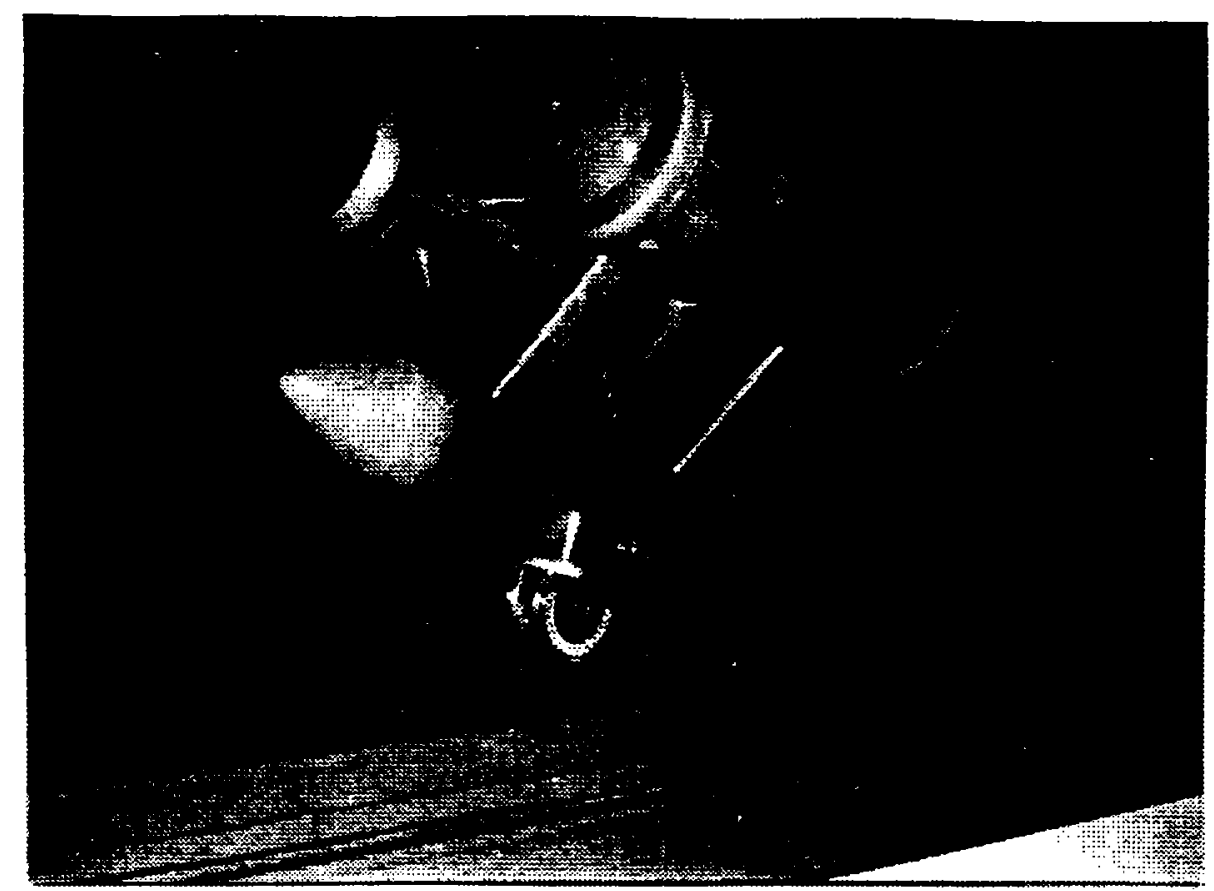

Figure 2-1: Scale Model of ABE vehicle

The model was attached to the towing carriage with an aluminum mount which allowed the pitch and yaw angle of the model to be varied with respect to the towing direction. The model could be set to have a pitch angle of \pm 90 degrees, a yaw angle of \pm 180 degrees or any combination of allowable pitch and yaw. All surfaces of the mounting flanges were rounded and their effects on the model hydrodynamics were considered to be small. The mounting assembly was rigidly attached to a fared aluminum strut which connected to the towing carriage.

The MIT Ocean Engineering Towing Tank can accommodate a tow run of approximately 75 feet $(23 \mathrm{~m})$. The tank is about 6 feet $(1.8 \mathrm{~m})$ wide and 4 feet $(1.2 \mathrm{~m})$ deep. The model was mounted upside down so that in the zero pitch condition the uppermost edge was 15 inches $(38 \mathrm{~cm})$ underwater. The lower edge of the model was approximately 20 inches $(51 \mathrm{~cm})$ above the bottom of the tank. The model was left positively buoyant since the addition of weight would have increased the loads during acceleration.

The forces and moments on the assembly were measured with a JR3 Universal Force-Moment Sensor System which has a rated capacity of $200 \mathrm{lbs}$ (890 N) force and 


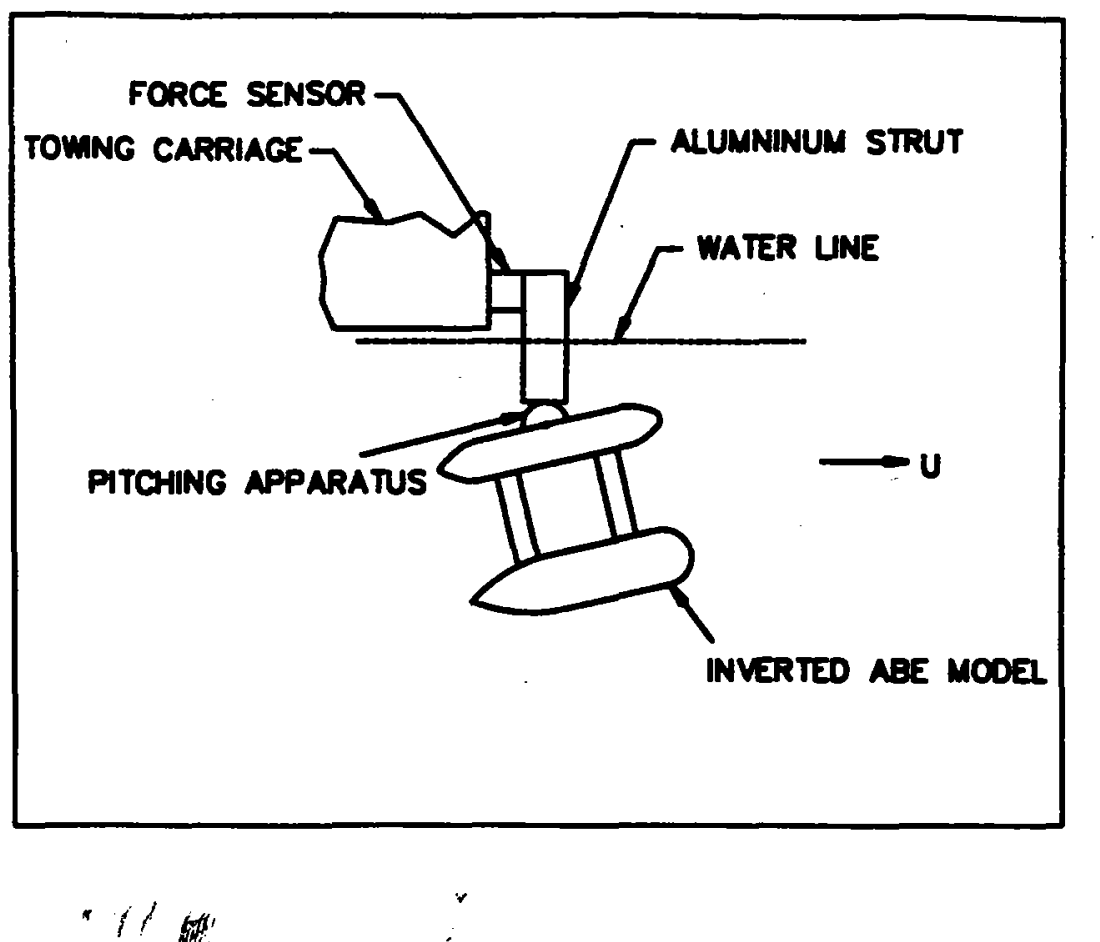

Figure 2-2: Experimental Setup

400 in-lbs (45 N-m) moment. The force gauge was mounted on the towing carriage just above the water level. The electronics enclosure and power supply were also mounted on the carriage. Figure 2-2 shows the complete setup.

The steady state velocity of the towing carriage was calibrated using a stopwatch and a measured distance. The carriage has a controller which allows the user to set the maximum acceleration and braking times which determine the dynamic response of the carriage. Since the transient velocity of the carriage could not be measured accurately, only steady state measurements were made.

The carriage could be moved safely at speeds up to 5 knots $(2.6 \mathrm{~m} / \mathrm{s})$. However, the time that the carriage velocity was in steady state was greatly reduced at the higher speeds. For proper Reynolds number scaling, the desired tow speed for the model was 3-6 knots (for an ABE vehicle speed of 1-2 knots). In some configurations of the model, the tow speed had to be kept low so that the rated capacity of the force gauge was not exceeded. The forces and moments returned by the force transducer were logged on a personal computer at a rate of 23 hertz. 


\subsection{Experimental Procedure}

The model was towed in its principal directions to get steady state drag force measurements. Several data runs were made for the forward direction with no pitch or

yaw angle. Data were recorded for speeds of 1, 3, 4 and 5 knots. Full reverse tests were done at 1 and 3 knots. Full sideways, up and down directions were studied at $\frac{1}{2}$ and 1 knot. The slower speeds were required in order to not damage the force transducer.

Time was allowed between runs so that the waves in the tank could settle. This was necessary since at the higher speeds a significant free surface wave effect was observed. To account for wave effects on the drag force measurements, a slow run for which there were no waves generated was done for each configuration.

To characterize the drag/lift relationship, the pitch angle was varied from $\mathbf{- 4 5}$ degrees to 45 degrees in 5 degree increments. For most of these runs, data were recorded for speeds of 1 and 3 knots. In some cases the moments were too high to safely tow the model at 3 knots. A similar yaw study was done to investigate the drag/side force relationship and can be found in [1]. The yaw data are not included here since in this report we are only concerned with the longitudinal plane.

\subsection{Results}

,

Figure 2-3 shows a typical data run at 3 knots. The moment about an axis perpendicular to the towing direction is plotted versus a sampling index. There is a startup transient spike which is quite large, a region of steady state moment measurement and a braking transient. The amplitude of the noise in the measurement was a function of the towing carriage speed and is attributed to carriage vibrations. The signal to noise ratio was found to increase roughly linearly with speed.

The frequency content of the noise was also found to vary with carriage speed. For speeds of 1 to 3 knots, the noise had a large frequency component near 3 hertz. For 4 knots there were components near 1.5 and 3 hertz and for 5 knots the noise 


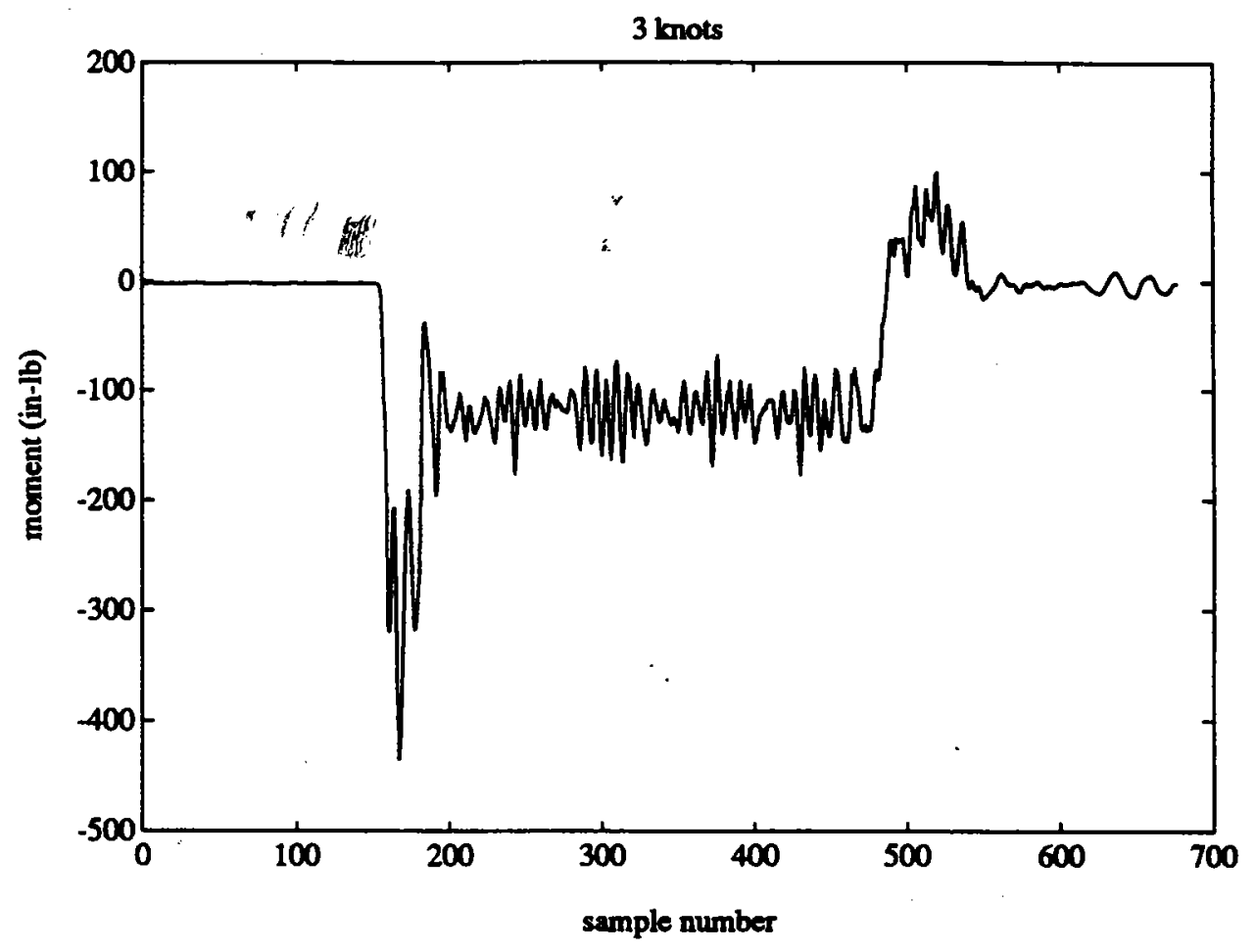

Figure 2-3: Typical Experimental Data 
is primarily at 1.5 hertz. It is assumed that these variations are a result of carriage imbalance and do not affect the mean data.

The average values of forces and moments were calculated for each run in a region of steady state. In some cases there was drift in the zero offset due to the large startup/stop loads on the sensor. These offsets were recorded and found to be less than 7 percent of the measurement in the worst (slowest) case. In the faster runs, the offset was found to be only 2 percent of the measurement.

The sensor hardware constrained the resolution of the force and moment measurements. The forces could be measured to within $0.05 \mathrm{lbf}(.22 \mathrm{~N})$ and the moments to 0.20 in-lbf (.023 N-m). For typical steady state values this corresponds to a maximum relative error of about 2 percent. The total measurement error due to sensor drift and resolution was less than 10 percent in the worst case.

The force measurement in the direction of towing was found to be partially saturated and could not be calibrated accurately. This was due to the large moment arm between the point of application of the force and the sensor. Fortunately, the moment measurement about the axis perpendicular to the towing direction was not contaminated. The vertical force was also unaffected by the large moment arm. The force that is in the towing direction can be estimated using a few simple assumptions regarding the forces and moments acting on the model.

The drag force on the model with zero pitch angle can de determined directly from the moment measurement by making an estimate of the moment arm to the drag force. The steady state forces and moments assumed to be acting on the model and assembly are shown in figure 2-4. The drag force is given by

$$
D=-\frac{M_{\text {measured }}}{a r m}
$$

where $D$ is the drag force, $M_{\text {measured }}$ is the moment measurement and arm is the moment arm. The drag depends directly on the moment arm which means, for example, an estimate for the arm that is 10 percent too high leads to a drag value that is 10 percent too low. 


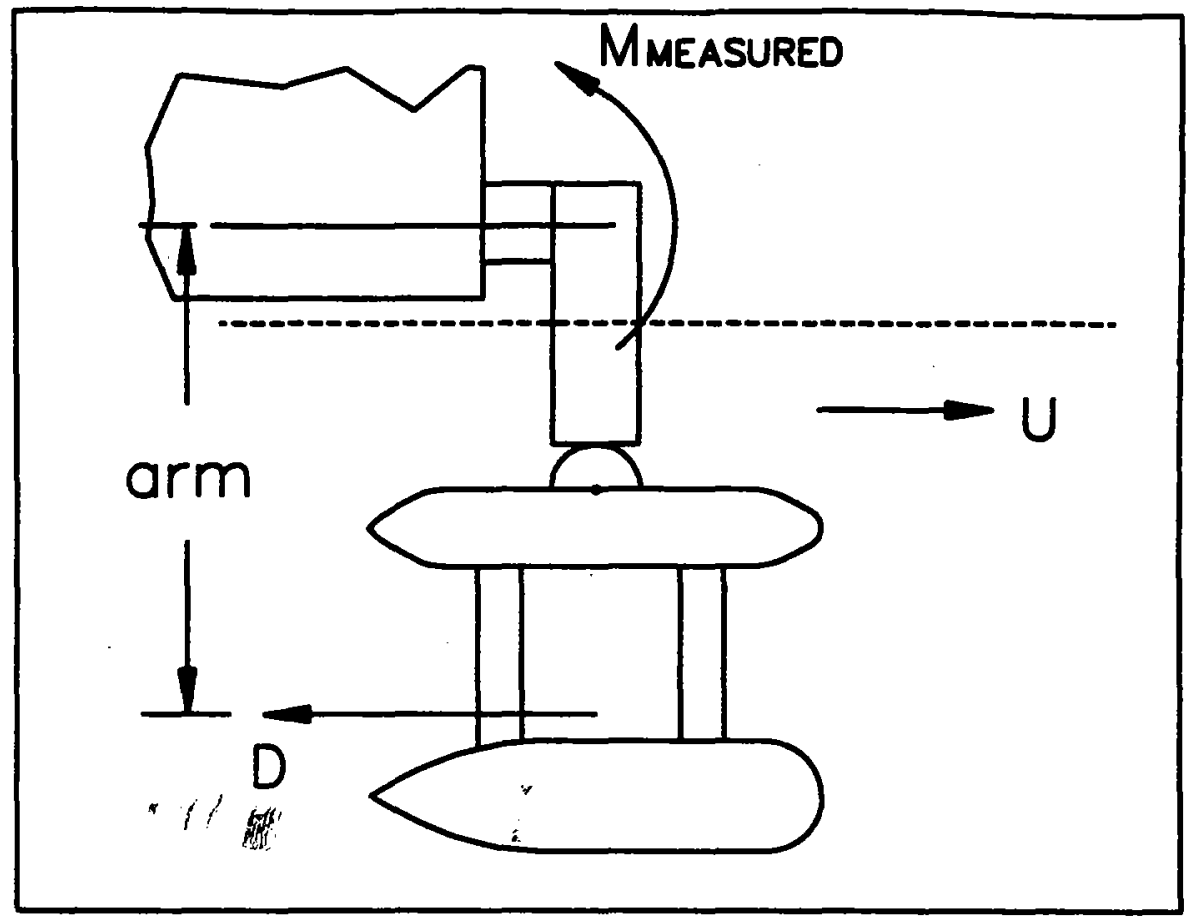

Figure 2-4: Forces and Moments on the Model

A reasonable estimate of the center of drag is the center of the projected area in the direction of motion. This corresponds to an arm of 25.44 inches $(65 \mathrm{~cm})$ for the model/strut assembly in a zero pitch angle condition.

The drag force in each direction is reduced to a drag coefficient with the formula

$$
C_{D}=\frac{D r a g}{\frac{1}{2} \rho A U^{2}}
$$

where $\rho=998 \mathrm{~kg} / \mathrm{m}^{3}$ is the density of water, $A$ is the projected frontal area in the direction of motion and $U$ is the model speed. The computed drag coefficients for each direction are averaged and presented in table 2.1 .

The forward drag coefficient is the same order of magnitude as the experimental values given in Hoerner [6]. The drag coefficients given are based on the square of the velocity without regard to any dependence on Reynolds number. It is assumed that the flow field is turbulent and that the drag coefficient at 1 knot is the same as the drag coefficient at 5 knots. Figure 2-5 shows the linear fit of the drag force in 
Table 2.1: Drag Coefficients

\begin{tabular}{|c|c|c|c|c|c|}
\hline Direction & $\begin{array}{c}\text { Moment Arm } \\
\text { (in) }\end{array}$ & $\begin{array}{c}\text { Area } \\
\left(\text { in }^{2}\right)\end{array}$ & $C_{D}$ & $\begin{array}{c}\text { Standard } \\
\text { Deviation }\end{array}$ & $\begin{array}{c}\text { Speeds } \\
(\text { knots })\end{array}$ \\
\hline \hline Forward & 25.44 & 97.91 & .0243 & .0040 & $1,3,4,5$ \\
\hline Sideways & 25.44 & 312.23 & .0757 & .0017 & $0.5,1$ \\
\hline Down & 18.25 & 337.96 & .0795 & .0028 & $0.5,1$ \\
\hline Up & 18.25 & 337.96 & .0767 & .002 & $0.5,1$ \\
\hline Reverse & 25.44 & 97.91 & .0486 & .0090 & 1,3 \\
\hline
\end{tabular}

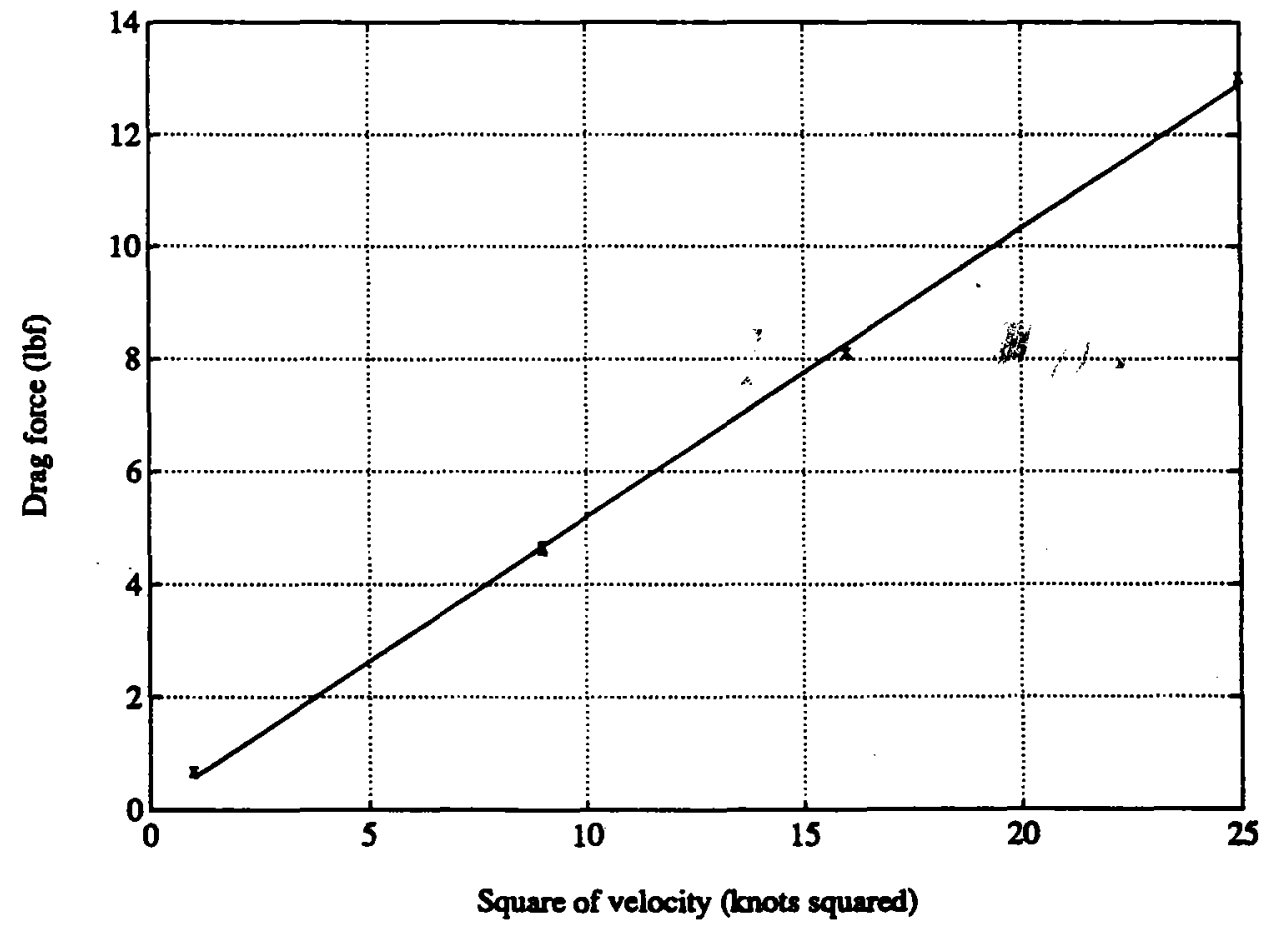

Figure 2-5: Square Law Drag Behavior 


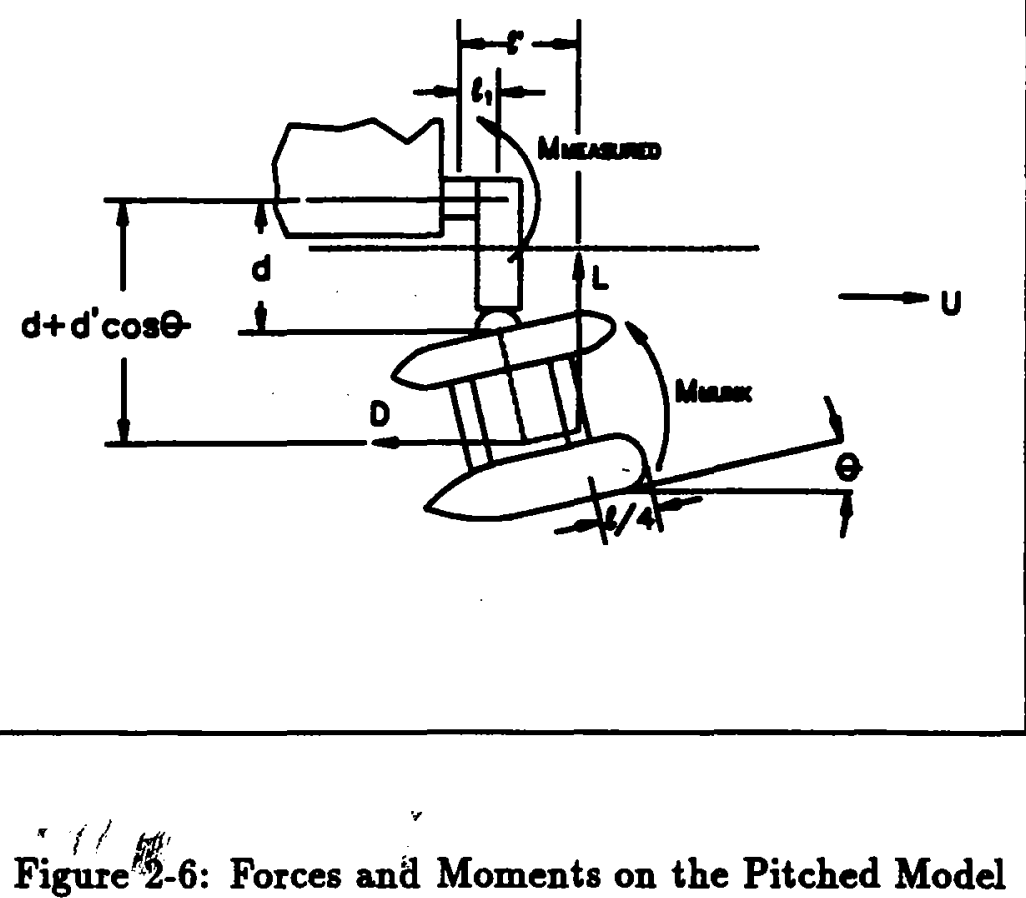

the forward direction versus the square of the velocity. It is evident from the linear behavior observed in this plot that any variation with Reynolds number is slight and can be ignored.

When the pitch angle of the model is varied, several more assumptions must be made to extract the drag force from the moment data. In this chapter, no distinction will be made between pitch angle and angle of attack since the model is fixed in space.

Figure 2-6 shows the locations of forces and moments assumed to act on the model. The drag force acts at the center of the frontal area and contributes a negative moment. A lift force is assumed to act at the effective one quarter chord point at the same $z$ location as the drag force. The lift force contributes a positive component to the measured moment.

When there is a nonzero angle of attack to the flow there is also a destabilizing Munk moment. The Munk moment is an inertial effect which can be written in terms of the principal added mass coefficients and the normal and tangential velocities. The local tangential velocity is

$$
u=U \cos \theta
$$


and the normal velocity is

$$
\boldsymbol{w}=U \sin \theta
$$

where $U$ is the total velocity and $\theta$ is the pitch angle. The Munk moment is given by

$$
M_{M \text { unk }}=\left(m_{33}-m_{11}\right) u w=\left(m_{33}-m_{11}\right) U^{2} \cos \theta \sin \theta
$$

$m_{33}$ is the added mass in the $z$ or vertical direction. It can be thought of as the fluid mass which is accelerated when the body moves vertically. $m_{11}$ is the fluid inertia associated with motion in the forward direction.

We can estimate the added mass values for the ABE model by using an approximation for prolate ellipsoids. Lamb [9] gives formulae for the principal added mass coefficients which depend on the length and diameter of the ellipsoidal bodies. The ABE model has three pods, each which have a shape similar to an ellipsoid. If we assume that there are no interference effects between the pods, superposition can be used to estimate the added masses.

Using the formulae given by Lamb, the added mass difference is

$$
m_{33}-m_{11}=1.1 \text { slugs }=16.05 \mathrm{~kg}
$$

This value is quite reasonable since the displaced mass of fluid is approximately 1.3 slugs $(19.0 \mathrm{~kg})$. Since the forward added mass should be verysmall for a streamlined body, the vertical added mass will dominate the added mass difference. For a cylinder, the added mass is equal to the mass of the displaced volume. It makes sense that the vertical added mass for the ABE model would be near to the displaced mass since it has cylindrical cross sections. It follows that the added mass difference should be slightly less than the displaced mass which is the above result.

From figure 2-6, the drag force can be written in terms of known or measured quantities

$$
D=-\frac{M_{\text {meanured }}-M_{M u n k}-L l^{\prime}}{d+d^{\prime} \cos \theta}
$$

where $M_{\text {measured }}$ is the measured moment at the sensor, $L$ is the measured lift force, 
Table 2.2: Sensitivity Study Maximum Errors

\begin{tabular}{|c|c|c|c|}
\hline $\begin{array}{c}\text { parameter } \\
\text { varied }\end{array}$ & variation & $\begin{array}{c}\text { maximum } \\
\text { error }\end{array}$ & $\begin{array}{c}\text { angle for } \\
\text { max error }\end{array}$ \\
\hline \hline$M_{\text {meanured }}$ & $10 \%$ & $5.3 \%$ & 45 \\
\hline$L$ & $10 \%$ & $4.5 \%$ & 5 \\
\hline$d^{\prime}$ & $10 \%$ & $-2.8 \%$ & 0 \\
\hline$l_{1}$ & $10 \%$ & $0.59 \%$ & 5 \\
\hline$M_{M_{\text {unk }}}$ & $10 \%$ & $-2.4 \%$ & 20 \\
\hline$M_{M_{\text {unk }}}$ & $20 \%$ & $-4.75 \%$ & 20 \\
\hline$M_{M_{\text {unk }}}$ & $30 \%$ & $-7.25 \%$ & 20 \\
\hline$M_{M_{\text {unk }}}$ & $40 \%$ & $-9.8 \%$ & 20 \\
\hline$M_{M_{\text {unk }}}$ & $50 \%$ & $-12 . \%$ & 20 \\
\hline
\end{tabular}

$l^{\prime}=l_{1}+d^{\prime} \sin \theta+\frac{l}{4} \cos \theta$ is the moment arm to the lift force, and the denominator is the moment arm to the drag force. The nominal values of the geometric parameters are

$$
\begin{array}{|c|c|}
\hline l_{1}=1 \text { in }=2.54 \mathrm{~cm} & l=24 \text { in }=60.96 \mathrm{~cm} \\
\hline d=18.25 \text { in }=46.36 \mathrm{~cm} & d^{\prime}=7.19 \text { in }=18.26 \mathrm{~cm} \\
\hline
\end{array}
$$

The sensitivity of the computed drag value to the above parameters was explored numerically by varying the uncertain geometric parameters and the measured moment by 10 percent. The Munk moment was varied up to 50 percent since it is believed to be the largest source of uncertainty. A relative error to the nominal drag value was computed for each case as a function of pitch angle. Table 2.2 is a summary of the maximum errors.

The sensitivity study shows that even for extreme deviations in the assumed Munk moment, the drag force only varies by a maximum of 12 percent. These results show that although the drag force measurement was unavailable, the drag force could be extracted from the moment measurement with reasonable accuracy.

The computed drag force as a function of pitch angle is plotted in figure 2-7. The $x$ markers correspond to a measurement at 3 knots and the $o$ markers are for 1 knot. 


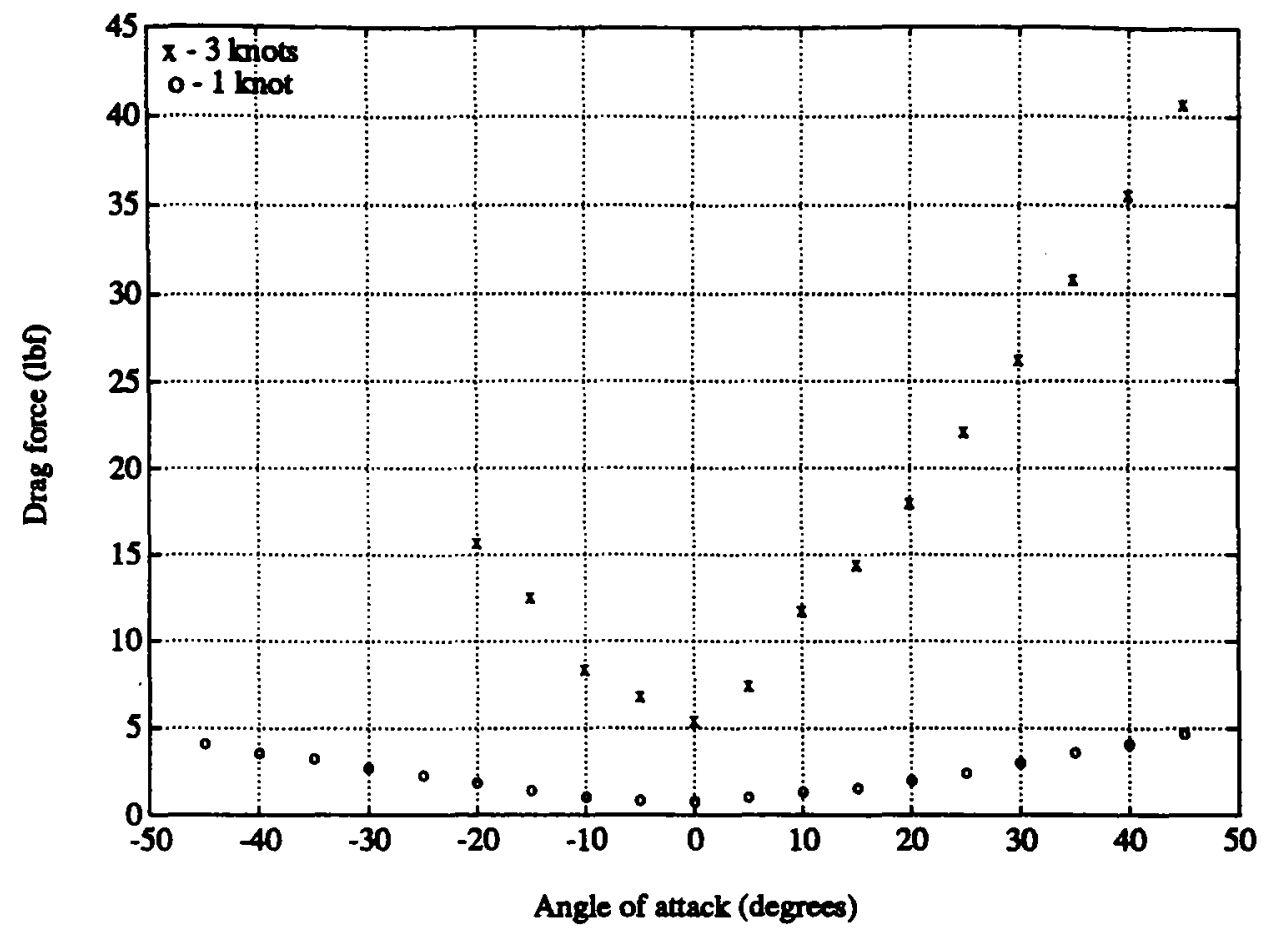

Figure 2-7: Measured Drag Forces as a Function of Pitch Angle

The drag force is normalized to a nondimensional drag coefficient with the formula

$$
C_{D}=\frac{\operatorname{Drag}}{\frac{1}{2} \rho A U^{2}}
$$

where $\rho$ is the density of water, $A$ is the constant frontal area of the scale model, and $U$ is the model velocity. The choice to normalize with respect to a constant area was made for simplicity. Since this model will be used for vehicle simulation and controller design, a drag coefficient based on constant area is more convenient than one based on a variable effective frontal area.

The drag coefficients as a function of the angle of attack are plotted in figure 2-8. The directional dependence on the sign of the pitch angle is expected since the ABE vehicle is not symmetric about any horizontal plane. Linear approximations to the 


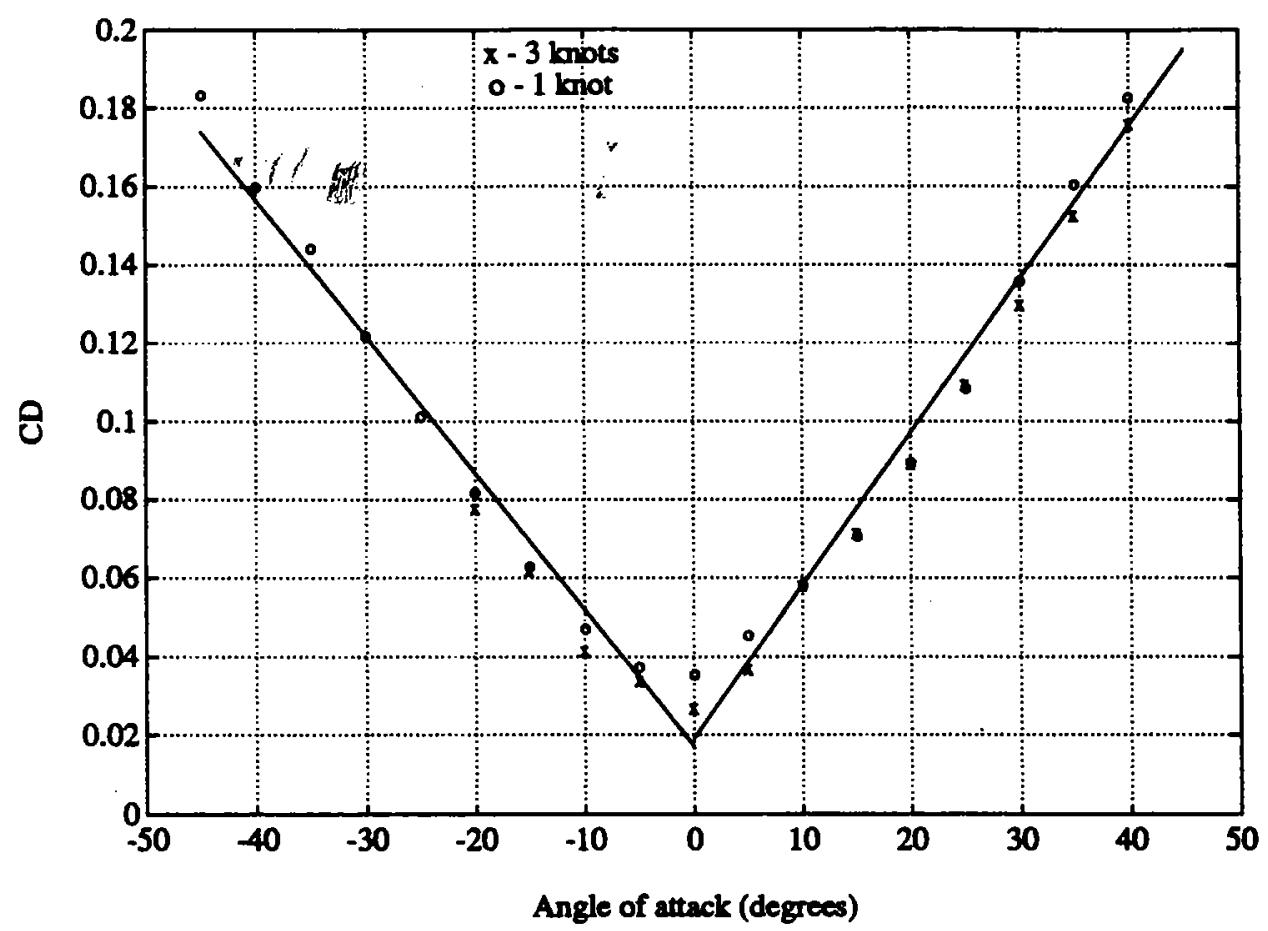

Figure 2-8: Drag Coefficient as a Function of Pitch Angle 
data are also shown. The linear fits are

$$
\begin{aligned}
& +\theta: C_{D}=3.908 \times 10^{-3} \cdot \theta+1.924 \times 10^{-2} \\
& -\theta: C_{D}=-3.487 \times 10^{-3} \cdot \theta+1.709 \times 10^{-2}
\end{aligned}
$$

where $\theta$ is in degrees. These linear approximations will be used later in vehicle simulation. The intention here is not to assert that the drag coefficient has a linear dependence on pitch angle. The decision to normalize the drag force with respect to a constant area gives this linear result. A different normalization would result in a different, perhaps nonlinear, curve.

The measured lift forces are plotted in figure 2-9. Again, $x$ 's are measured values at 3 knots and o's are for 1 knot. There is an obvious change in the lift behavior between 5 and 10 degrees. This change is more evident in the plot of a normalized lift coefficient as a function of pitch angle. The lift coefficient is computed using the formula

$$
C_{L}=\frac{L i f t}{\frac{1}{2} \rho A U^{2}}
$$

where the parameters are the same as for the drag coefficient except for the area $A$. For the lift case, a variable projected bottom area is used. This was chosen for normalization since the data fit a linear curve. A constant area normalization is also a reasonable way of presenting the data." However, for purposes of simplicity for simulation, linear fits were desirable.

Figure 2-10 shows the lift coefficient variation with pitch angle and linear approximations to the behavior. As noted before, there is a significant change in behavior near 5 degrees. At low angle of attack there is a rapid increase in lift force as the angle is increased. Then, there is a stall condition where there is no increase in force for added angle of attack. The lift coefficient then resumes a less steep linear behavior at around \pm 20 degrees.

The linear approximations on the lift coefficient plot are given by

$$
\operatorname{low} \theta: C_{L}=2.809 \times 10^{-4} \cdot \theta-3.770 \times 10^{-5}
$$




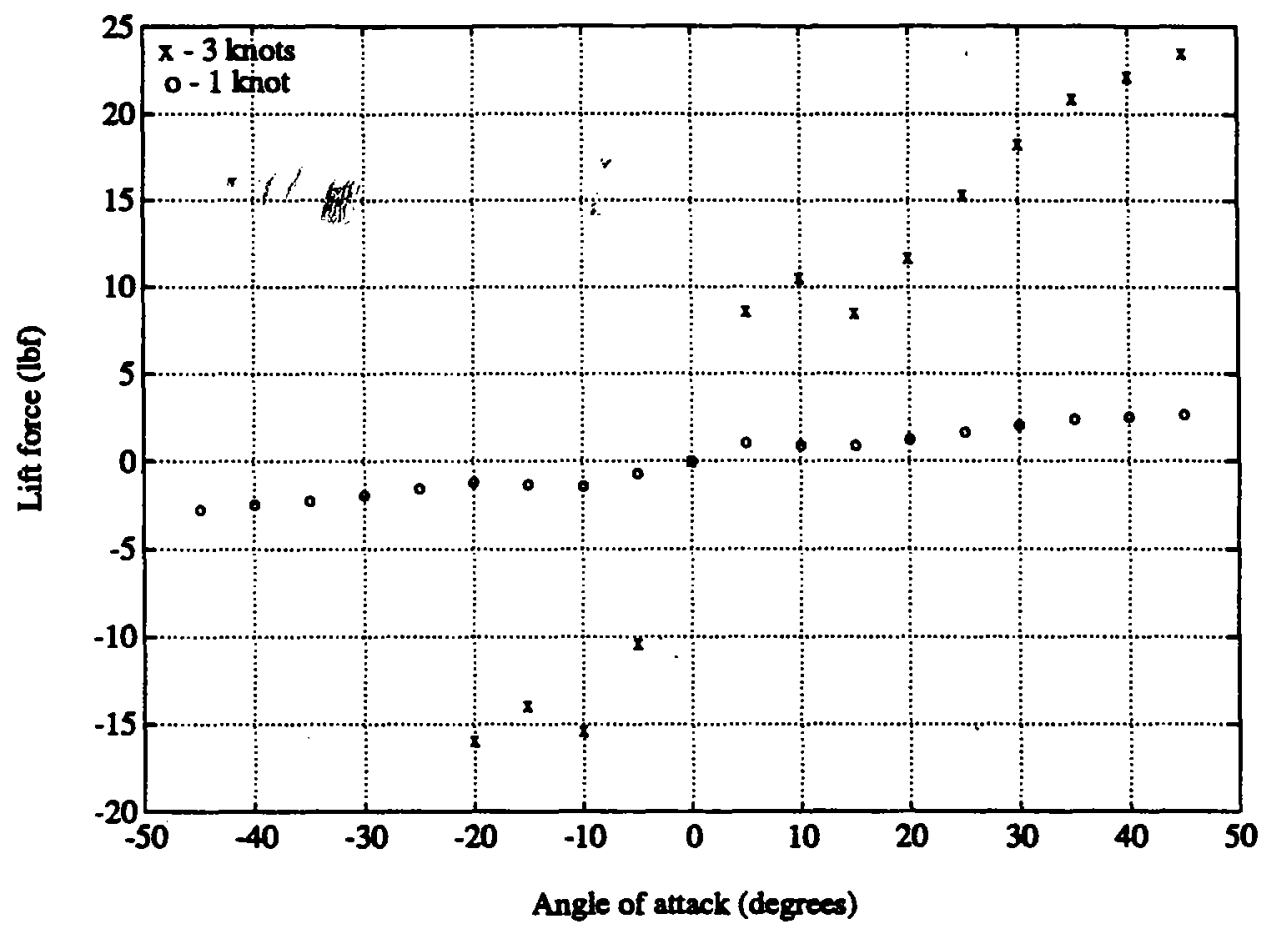

Figure 2-9: Lift Force as a Function of Pitch Angle 


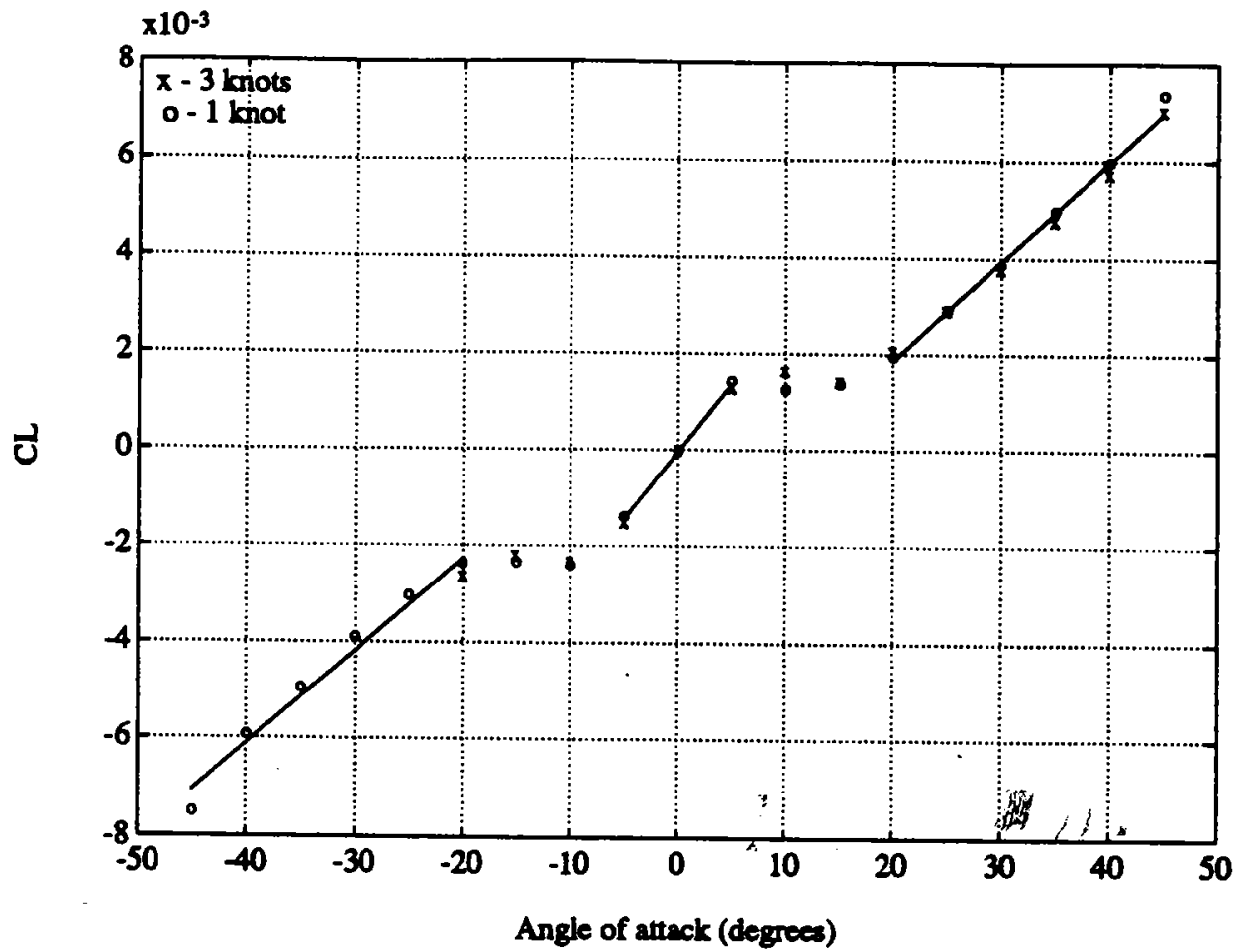

Figure 2-10: Lift Coefficient as a Function of Pitch Angle 


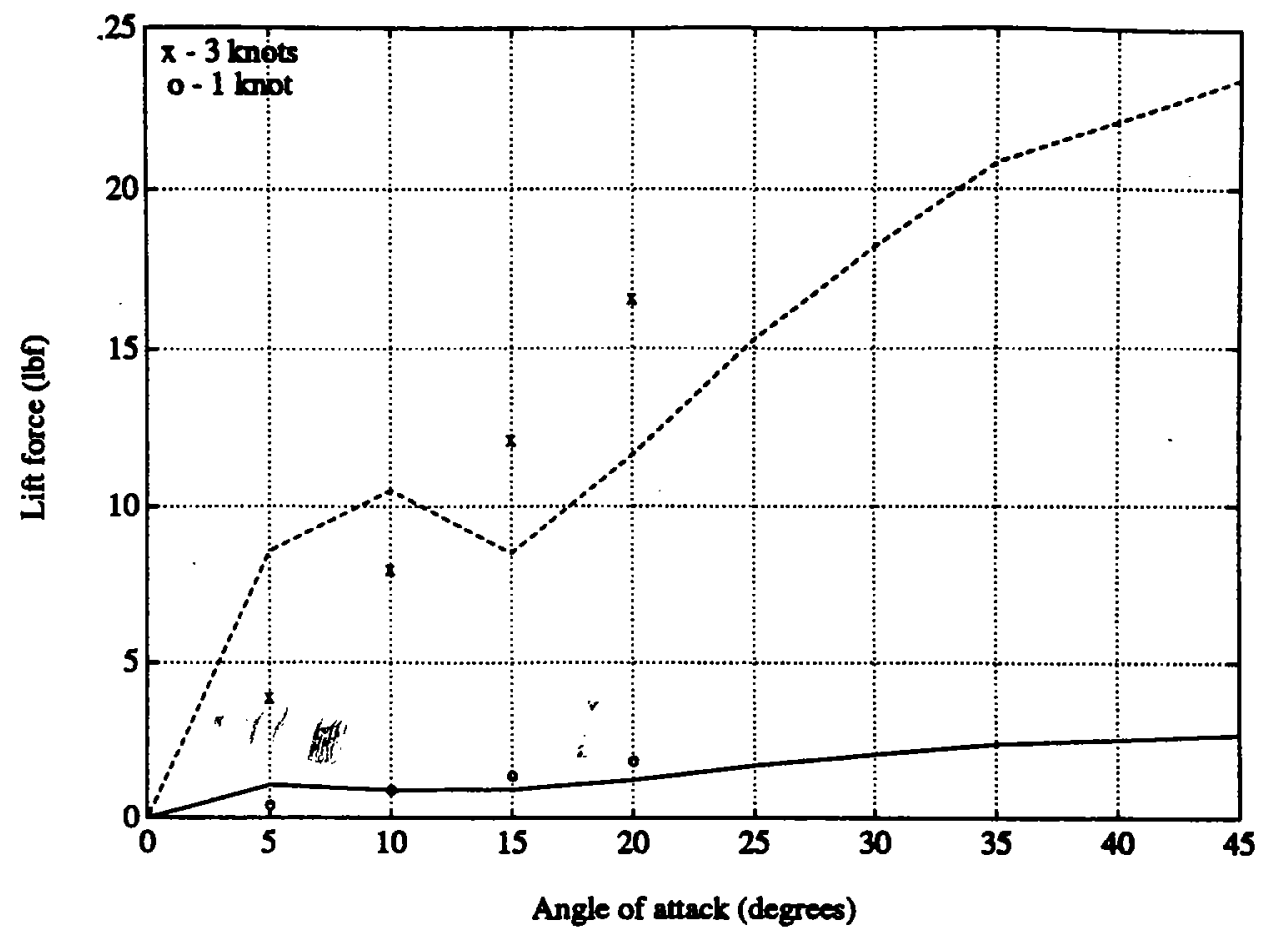

Figure 2-11: Measured Lift Forces Compared to Data for Bodies of Revolution

$$
\begin{aligned}
& \text { high }+\theta: C_{L}=2.047 \times 10^{-4} \cdot \theta-2.217 \times 10^{-3} \\
& h i g h-\theta: C_{L}=1.919 \times 10^{-4} \cdot \theta+1.566 \times 10^{-3}
\end{aligned}
$$

The lift force data are in general agreement with experimental results given in reference [7] for similar bodies of revolution. From reference [7], the lift data for bodies with similar slenderness ratios to the ABE pods are added together. The lifting behavior of the horizontal struts are added in as if they were flat plates. These modified experimental results are compared the lift forces measured in the towing tank in figure 2-11. The dashed and solid lines are the measured lift forces for the ABE model.

There is good agreement between the modified experimental data from [7] and the measured ABE lift forces. However, only the ABE model testing results reveal the marked stall behavior at low angles of attack. This behavior could not have 


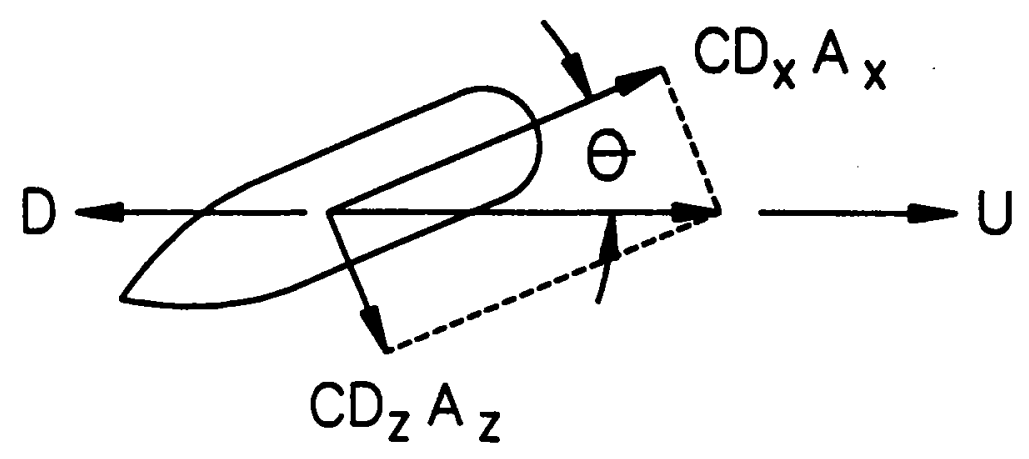

Figure 2-12: Model A

been predicted otherwise. The measured data also account for the interference effects between the three pods. The [7] data overpredict the actual lift force. This makes intuitive sense because the mechanism for lift is cross flow drag. There is a reduction in the cross flow drag since the pods block each other with respect to the flow when there is an angle of attack. The comparison with [7] validates the lift results and demonstrates the unique lifting properties of the $A B E$ vehicle.

\subsection{Comparison with Simple Drag Models}

It is interesting to note how the measured data compare with some models of drag and lift behavior for pitched bodies. Two simple models will be considered.

Model $\mathrm{A}$ is a drag only model which decomposes the forward and vertical drag coefficients and their two areas. A model of this type would be suited to a bluff body with no lifting surfaces. Figure 2-12 is a schematic diagram of the model. The projected $C_{D} \cdot A$ in the direction of motion is 


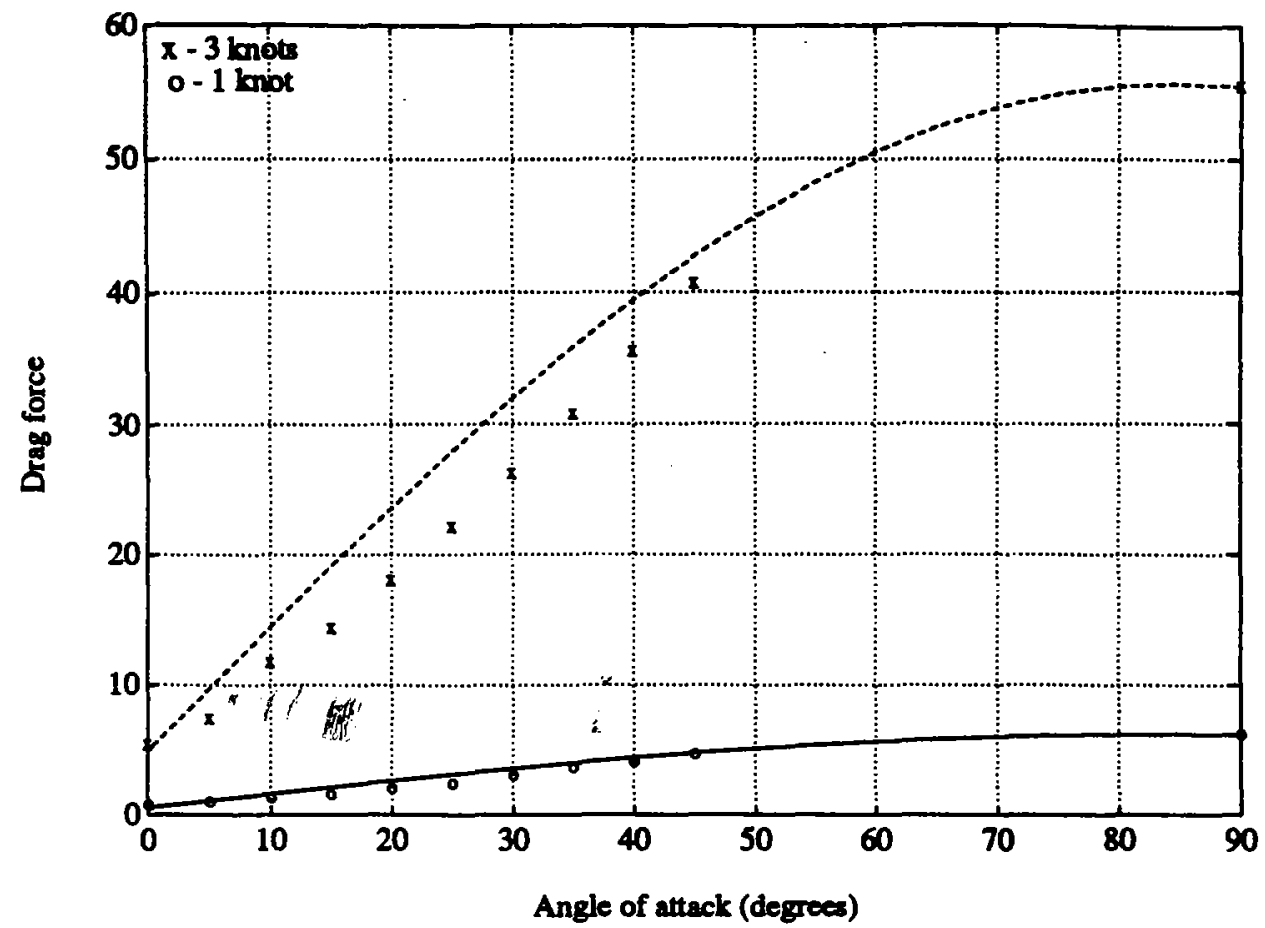

Figure 2-13: Performance of Model A

$$
C_{D} \cdot A=C_{D_{z}} A_{z} \cos \theta+C_{D_{z}} A_{z} \sin \theta
$$

and the drag force is given by

$$
D=\frac{1}{2} \rho C_{D} \cdot A U^{2}
$$

in the opposite direction as the velocity $U$. Figure 2-13 shows how this model does in predicting the drag force. The measured data are plotted along with the model prediction. The model generally overestimates the actual drag force. The maximum error is approximately 11 percent compared to the maximum drag measurement. This model cannot predict a lift force.

The second model is shown in figure 2-14. The velocity is considered to have two 


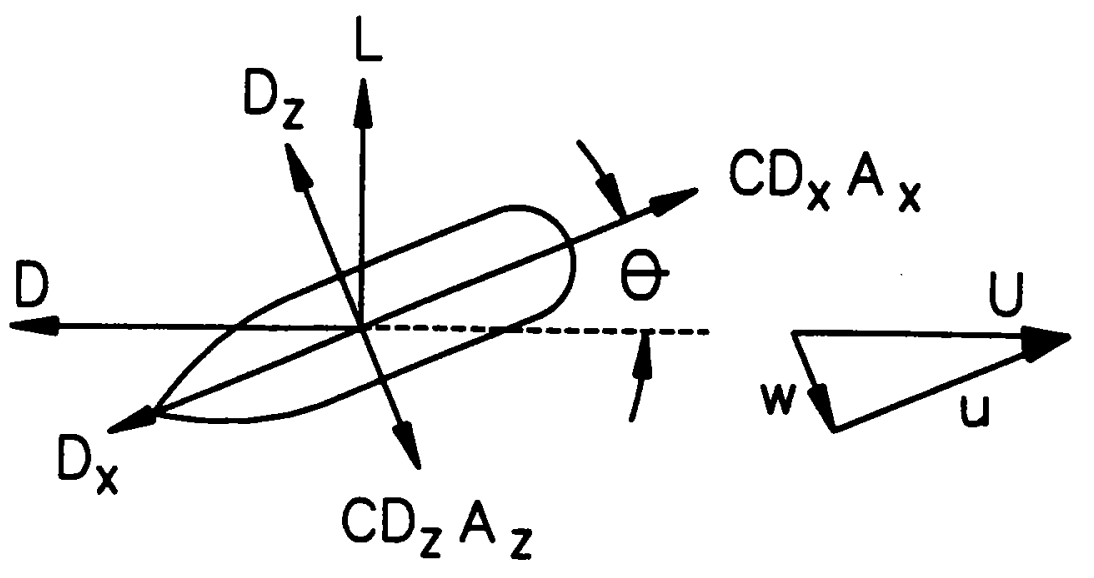

Figure 2-14: Model B

parts, one in the forward direction and one in the vertical. Each velocity component exerts a force which depends on the drag coefficient and area in that direction. This model is suited to a slender body where there is both drag and lift. The drag force components are

$$
\begin{aligned}
& D_{z}=\frac{1}{2} \rho C_{D_{z}} A_{z} v_{z}^{2}=\frac{1}{2} \rho C_{D_{z}} A_{z} U^{2} \cos ^{2} \theta \\
& D_{z}=\frac{1}{2} \rho C_{D_{z}} A_{z} v_{z}^{2}=\frac{1}{2} \rho C_{D_{z}} A_{z} U^{2} \sin ^{2} \theta
\end{aligned}
$$

Now, the drag and lift forces as defined before can be written in terms of these drag components

$$
\begin{gathered}
D=D_{z} \cos \theta+D_{z} \sin \theta \\
L=-D_{z} \sin \theta+D_{z} \cos \theta
\end{gathered}
$$

The drag performance for model $B$ is shown in figure 2-15. The model severely 


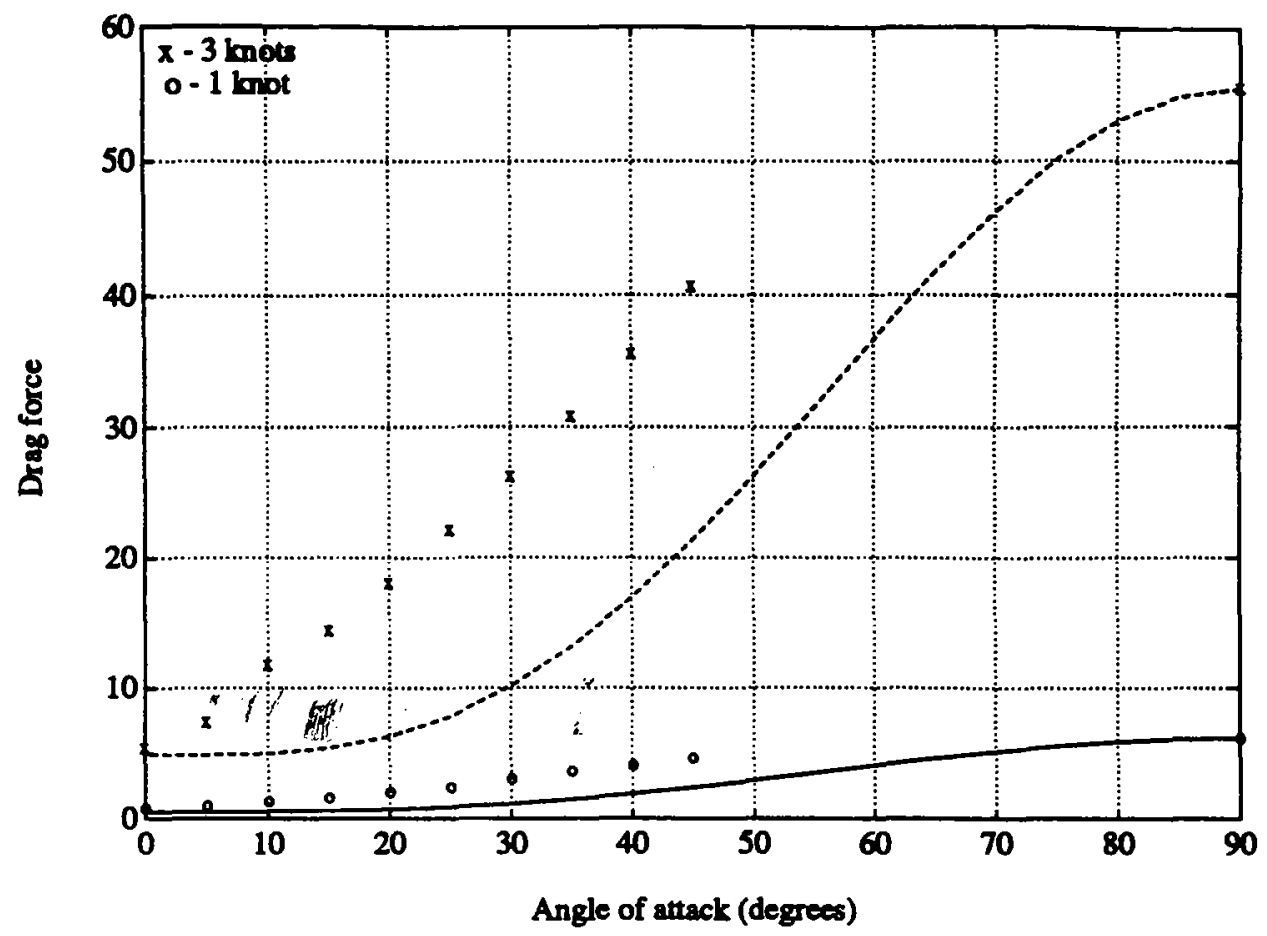

Figure 2-15: Model B-Drag Force

underpredicts the actual drag force with errors as high as 35 percent compared to the maximum measurement. The B lift model is shown in figure 2-16. It also underestimates the actual force and cannot model the region of stall.

The comparison of the ABE experimental data with these two models illustrates an important point. The ABE vehicle is neither a bluff body nor a slender body. It has the characteristics of both. The foil shaped struts contribute a significant lift force which is a property of a slender body. However, the drag behavior is typical of a bluff body as shown in figure 2-13. Thus, the experimental results are important since they contain both aspects of the behavior. 


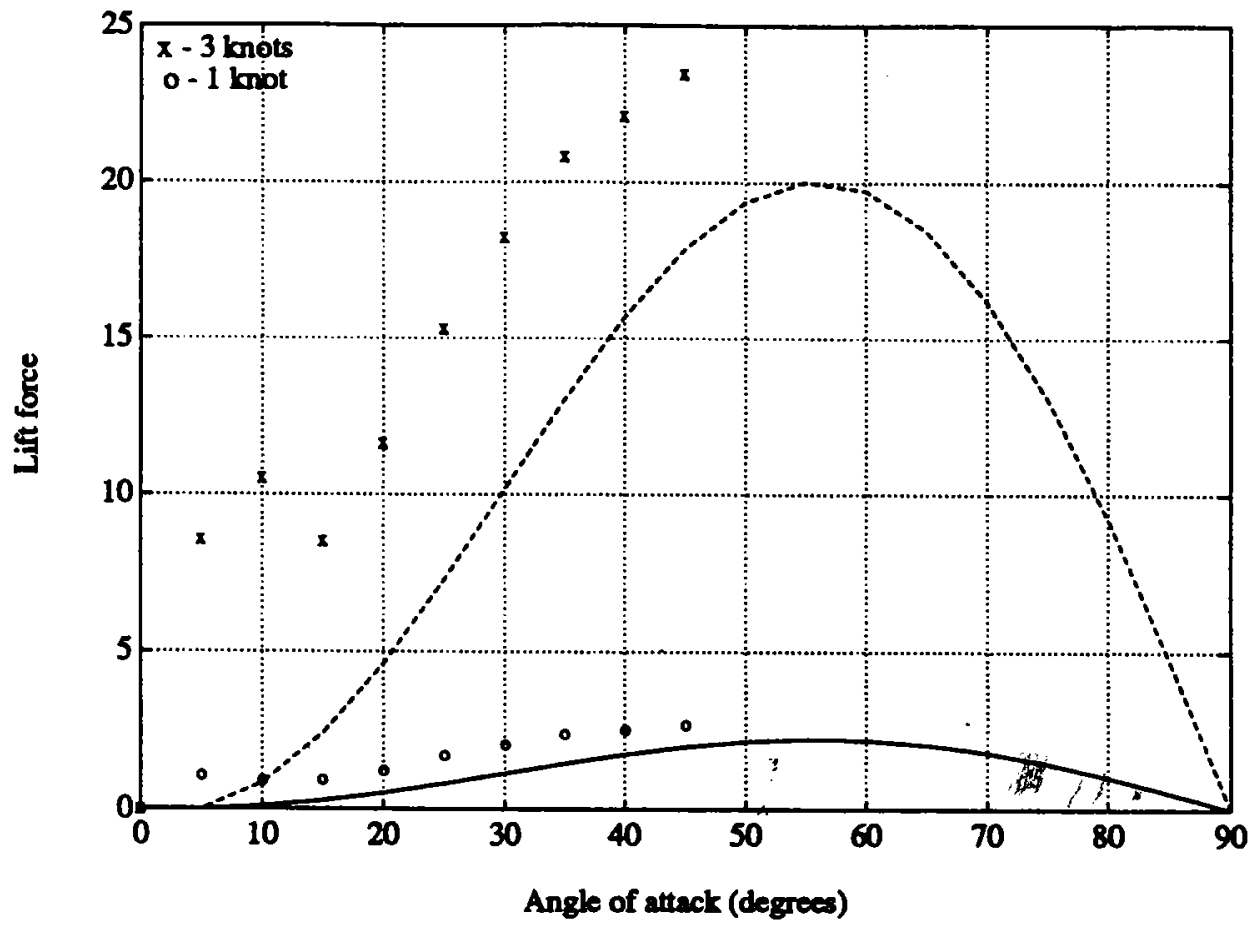

Figure 2-16: Model B-Lift Force 


\section{Chapter 3}

\section{The ABE Model}

\subsection{Introduction}

In this chapter, a model of the ABE vehicle dynamics is developed for the longitudinal plane of motion. Established techniques are used along with the results of the scale model experiments. An experimentally verified model of the vehicle thrusters is included.

\subsection{Coordinate System}

A standard naval architecture body coordinate system is used with the $\mathrm{x}$-axis in the forward direction, the $y$-axis pointing starboard and the z-axis pointing down. The pitch angle $\theta$ is positive when the nose is raised. The surge velocity is the velocity in the forward or $x$ direction and is called $u$. Similarly, the heave velocity is in the z-direction and is denoted by $w$. The time derivative of the pitch angle is the pitch velocity which is called $q$. The definitions of the body coordinate system are illustrated in figure 3-1.

The motions in the longitudinal plane, i.e., $x, z$ and $\theta$ are assumed to be decoupled from motions in the lateral plane. This assumption is quite valid for symmetric bodies like the $\mathrm{ABE}$ vehicle. This means that rolling, yawing and swaying motions do not influence the surge, heave or pitch dynamics. Similarly, motions in the longitudinal 


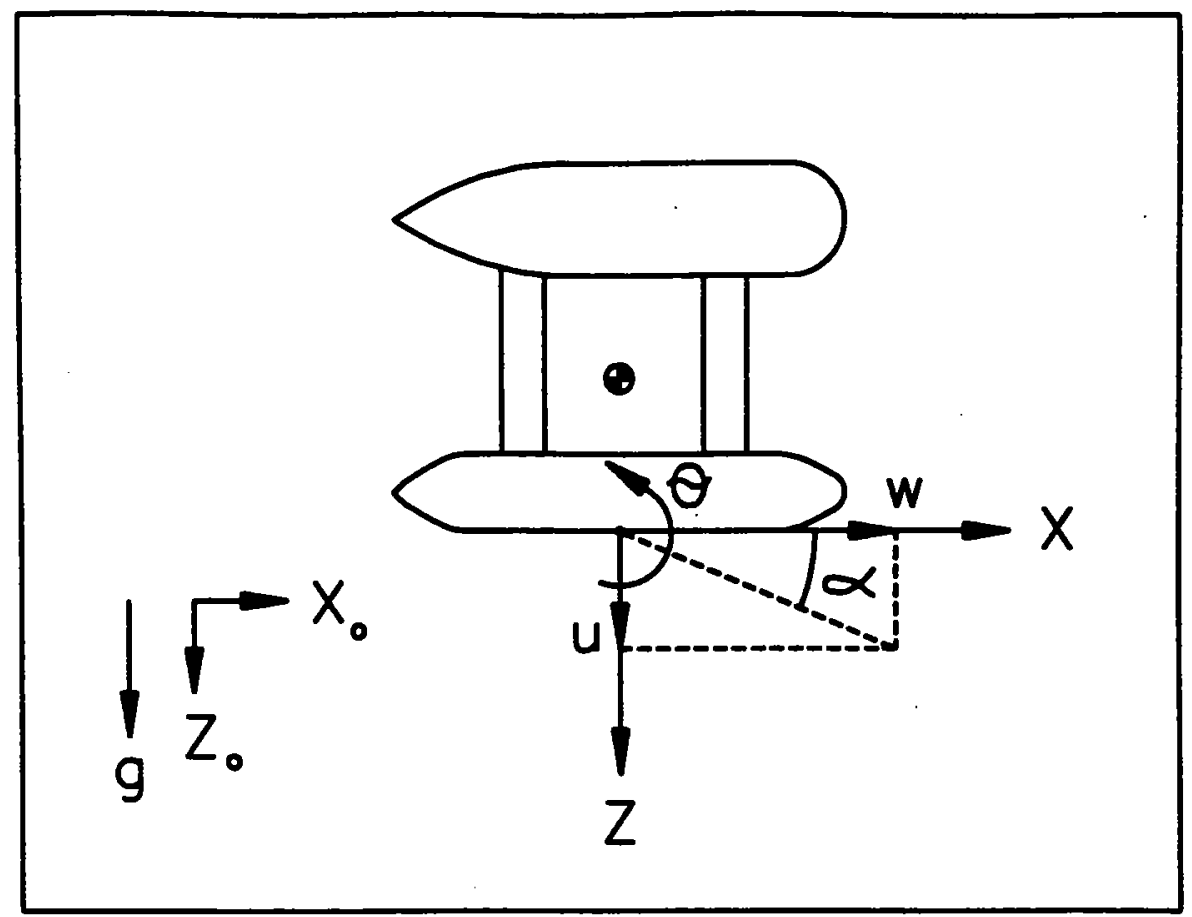

Figure 3-1: Body Coordinate System Definitions

plane do not induce a lateral response. Although this is an approximation, it will suffice for the ABE problem since multi-axis trajectories are not required.

\subsection{Formulation of the Equations of Motion .}

The hydrodynamic forces on the vehicle act in relation to the vehicle body coordinate system whereas the gravity/buoyancy forces act in an inertial reference frame. The equations of motion are formulated in the moving and rotating body coordinate system. Newton's Law for rigid body motion can be written as

$$
\begin{aligned}
& \Sigma \vec{F}=\frac{d}{d t}\left(m \overrightarrow{V_{c g}}\right) \\
& \Sigma \vec{M}=\frac{d}{d t}(I \vec{\Omega})_{c g}
\end{aligned}
$$

where $m$ is the body mass, $\vec{V}_{c g}$ is the velocity of the center of gravity in an inertial reference frame, $I$ is the inertial tensor about the center of gravity and $\vec{\Omega}$ is the 
angular velocity of the body.

To convert these equations to a body reference frame we define the following body coordinate system quantities. First, the linear and angular body velocity vectors are

$$
\begin{gathered}
\vec{U}_{b}=u \vec{i}+w \vec{k} \\
\vec{\Omega}=q \vec{j}
\end{gathered}
$$

The forces and moments are written as

$$
\begin{gathered}
\Sigma \vec{F}=X \vec{i}+Z \vec{k} \\
\Sigma \vec{M}=M \vec{j} \\
\overrightarrow{R_{g}}=x_{g} \vec{i}+z_{g} \vec{k}
\end{gathered}
$$

where $\vec{R}_{g}$ defines the location of the center of gravity with respect to the body coordinate system. If coriolis and centripetal forces due to the earth's rotation are neglected, Newton's law in component form becomes

$$
\begin{gathered}
m\left[\dot{u}+w q-x_{g} q^{2}+z_{g} \dot{q}\right]=X \\
m\left[\dot{w}-u q-z_{g} q^{2}-x_{g} \dot{q}\right]=Z \\
I_{\nu} \dot{q}+m\left[z_{g}(\dot{u}+w q)-x_{g}(\dot{w}-u q)\right]=M
\end{gathered}
$$

where the dot denotes the time derivative. There are also three auxiliary equations which transform body coordinate quantities to the inertial coordinate system. These are

$$
\begin{gathered}
\dot{\theta}=q \\
\dot{X}_{0}=u \cos \theta+w \sin \theta
\end{gathered}
$$




$$
\dot{Z}_{o}=-u \sin \theta+w \cos \theta
$$

where $X_{0}$ and $Z_{0}$ are the horizontal and vertical displacements of the body coordinate system origin in an inertial reference frame.

\subsection{Modelling the Forces on the Vehicle}

\subsubsection{Gravity and Buoyancy Forces}

The equations of motion are formulated in the body coordinate frame. There are two forces which act in the inertial frame: gravity and buoyancy forces. In general, the $A B E$ vehicle will be neutrally buoyant. The possibility of extra buoyancy or weight will be included in the model in order to remain as general as possible.

The buoyancy center is assumed to be in the center of the rehicle directly above the center of gravity. In the body coordinate system, the x-coordinates of the center of buoyancy and center of gravity are zero. The possibility of variable buoyancy or variable ballast will be included in the model so the terms which involve $x_{b}$ and $x_{g}$ are kept. The forces and moment can be derived with simple geometry to be

$$
\begin{gathered}
X_{b \text { boy }}=-(W-B) \sin \theta \\
Z_{\text {buoy }}=(W-B) \cos \theta \\
M_{\text {buoy }}=-\left(x_{g} W-x_{b} B\right) \cos \theta-\left(z_{g} W-z_{b} B\right) \sin \theta
\end{gathered}
$$

where $W$ is the dry weight of the vehicle, $B$ is the buoyancy force, $z_{b}$ is the distance to the center of buoyancy and $z_{g}$ is the distance to the center of gravity.

\subsubsection{Fluid Inertial Forces}

The forces on the vehicle must include the effects the fluid particles surrounding the vehicle. These fluid inertial forces can be derived using a potential flow analysis which assumes that the fluid is infinite, inviscid and irrotational. Newman [10] gives 
a detailed derivation.

The resulting forces and moments can be written in terms of an added mass tensor. The physical interpretation of the added mass $m_{i j}$ is that it is the fluid mass which has momentum in the $j^{\text {th }}$ direction due to body motion in the $i^{\text {th }}$ direction. The added mass tensor is symmetric and is dependent on the coordinate system used. For a port/starboard symmetric body like the ABE vehicle, there are 12 independent added mass elements. For the longitudinal plane, a simplified added mass tensor with 6 independent elements can be written,

$$
m_{i j}=\left[\begin{array}{lll}
m_{11} & m_{13} & m_{15} \\
m_{31} & m_{33} & m_{35} \\
m_{51} & m_{53} & m_{55}
\end{array}\right]
$$

where 1 is the surge $(x)$ direction, 3 is the heave (z) direction and 5 is the pitch direction. The fluid inertial forces are given by

$$
\begin{gathered}
X_{f l u i d}=-m_{11} \dot{u}-m_{13} \dot{w}-m_{15} \dot{q}-m_{31} u q-m_{33} w q-m_{35} q^{2} \\
Z_{f l u i d}=-m_{31} \dot{u}-m_{33} \dot{w}-m_{35} \dot{q}+m_{11} u q+m_{13} w q+m_{15} q^{2} \\
M_{\text {fluid }}=-m_{51} \dot{u}-m_{53} \dot{w}-m_{55} \dot{q}-m_{13} w^{2}-m_{15} q w \\
+m_{31} u^{2}+m_{35} u q+\left(m_{33}-m_{11}\right) u w
\end{gathered}
$$

These equations include the well known added mass force, the Munk moment and also the coupled effects which arise because the vehicle coordinate system is not stationary.

These equations are rewritten in terms of hydrodynamic coefficients to conform with the literature [2], [4] and [5]. The symmetry of the added mass matrix is taken into account to simplify the number of coefficients 


$$
\begin{aligned}
& X_{f l w i d}=X_{\dot{v}} \dot{u}+X_{\dot{w}} \dot{w}+X_{\dot{q}} \dot{q}+X_{\dot{w}} u q+Z_{\dot{w}} w q+Z_{\dot{q} q} q^{2} \\
& Z_{f l u \dot{d}}=X_{\dot{w} \dot{u}}+Z_{\dot{w}} \dot{w}+Z_{\dot{q}} \dot{q}-X_{\dot{u}} u q-X_{\dot{w}} w q-X_{\dot{q}} q^{2} \\
& M_{\text {fluid }}=X_{\dot{q}} \dot{u}+Z_{\dot{q}} \dot{w}+M_{\dot{q}} \dot{q}+X_{\dot{w}} w^{2}+X_{\dot{q}} q w-X_{\dot{w}} u^{2}-Z_{\dot{q}} u q-\left(Z_{\dot{w}}-X_{\dot{u}}\right) u w
\end{aligned}
$$

The principal added mass coefficients can be estimated using Lamb's [9] formulae for prolate ellipsoids. The assumptions are made that the added masses are independent for the three pods of the ABE vehicle and that superposition holds. Each pod is approximated by an ellipsoid, its added masses are calculated using Lamb's formulae, and then the results are summed. This is a reasonable approximation since the ABE pods are similar in shape to ellipsoids. Unmodelled interference effects may be present in the vertical (z) direction but are not expected for the forward $(x)$ direction since the body is very streamlined.

The resulting values for the $\mathrm{ABE}$ vehicle are

$$
\begin{array}{|l|l|l|}
\hline X_{\dot{u}}=-m_{11}=-38 & \mathrm{~kg} & Z_{\dot{v}}=-m_{3 s}=-454 \mathrm{~kg} \\
\hline
\end{array}
$$

In a similar manner, the added inertia for the pitch axis can be calculated as if the added inertia is considered to be comprised of three parts, one from each pod. Using the formula for an ellipsoid given by Lamb, the result is

$$
M_{q}=-m_{\mathrm{Bb}}=-997 \mathrm{~kg}-\mathrm{m}^{2}
$$

A symmetry argument can be used to ascertain that the coefficient $Z_{\dot{q}}$ is negligible. From an added mass point of view, the force associated with $Z_{\dot{q}}$ is a vertical force which is due to a pitching motion, or by reciprocity, it is a pitching moment due to vertical motion. Since the body coordinate system is in the center of the vehicle with respect to the $x$-aqxis, a vertical motion will only induce pitching if there is a nose/tail asymmetry. For the ABE vehicle, this would be small so we have

$$
Z_{\dot{q}}=0
$$


By a similar symmetry argument, the coefficient $X_{\dot{w}}$ can also be considered negligible. If this coefficient were nonzero, an acceleration in the $x$ direction would cause a force in the $z$ direction. For the ABE geometry, this is unlikely so for simplicity we set

$$
X_{\dot{w}}=0
$$

The last of the six independent inertia coefficients is $X_{\mathbf{q}}$. This coefficient cannot be ignored because for the body coordinate system chosen, there is a pitching moment induced for forward acceleration. If we consider the induced moment as the forward added mass force multiplied by an appropriate moment arm, the coefficient is

$$
\text { *1t } \quad \vdots \boldsymbol{X}_{\dot{q}}=\boldsymbol{X}_{\dot{\mathrm{v}} z_{\mathrm{co}}}
$$

where $z_{\mathrm{co}}$ is the $z$ distance to the center of volume, positive down. For the ABE, $z_{\mathrm{ev}}=-1.02$ meters so we have

$$
X_{\dot{q}}=38 \mathrm{~kg}-\mathrm{m}
$$

\subsubsection{Viscous Forces}

The viscous forces on the vehicle include a drag force in the direction of the vehicle velocity, a lift force perpendicular to the velocity and a pitching moment. The lift and moment are considered to be viscous effects since we are only concerned with the lift and moment induced by cross-flow drag, not by circulation of the flow as for a hydrofoil.

The drag/lift model used here is the one developed using scale model experiments as described in chapter 2 . The drag and lift coefficients determined earlier can be applied directly to the full scale vehicle since they are nondimensionalized. However, it must be noted that the scale model measurements were made in steady state conditions. By applying those results here, the transient nature of the viscous forces is neglected. 


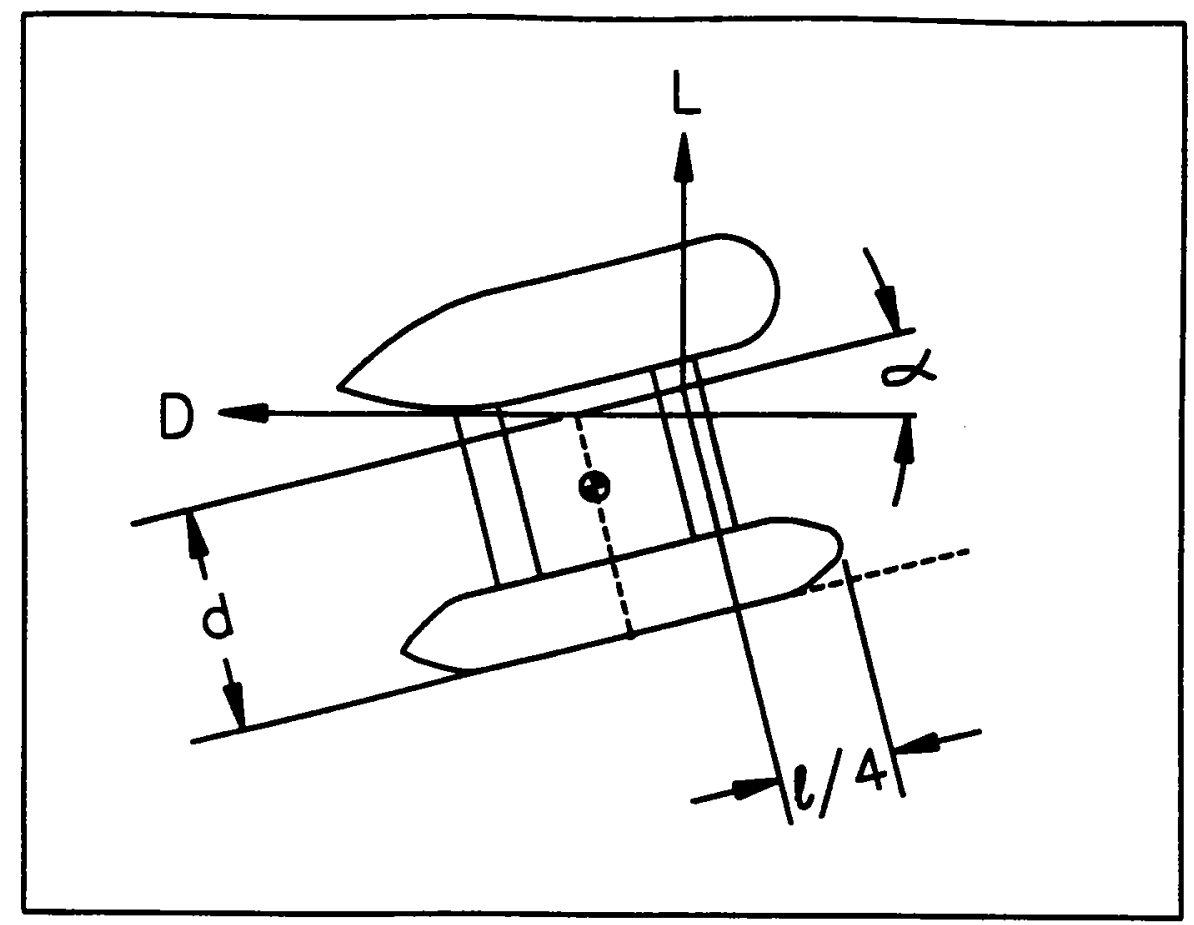

Figure 3-2: Assumed Forces and Moments on the Vehicle

The drag and lift forces can be transformed to the body coordinate system by a simple transformation. For a given angle of attack $\alpha=\tan ^{-1}\left(\frac{w}{u}\right)$, the forces are

$$
\begin{aligned}
& X_{\text {viscous }}=-D \cos \alpha+L \sin \alpha \\
& Z_{\text {viscoles }}=-D \sin \alpha-L \cos \alpha
\end{aligned}
$$

where the drag and lift forces are

$$
\begin{gathered}
D=\frac{1}{2} \rho C_{D}(\alpha) A_{\alpha} U^{2} \\
L=\frac{1}{2} \rho C_{L}(\alpha)\left(A_{2} \cos \alpha-A_{z} \sin \alpha\right) U^{2}
\end{gathered}
$$

To formulate a model for the moment due to the drag and lift forces, some assumptions must be made regarding the points of application of the forces. Figure 3-2 shows the assumed locations of the points of application of the forces. The drag acts at the center of the frontal area at a distance $d$ above the body coordinates origin. 


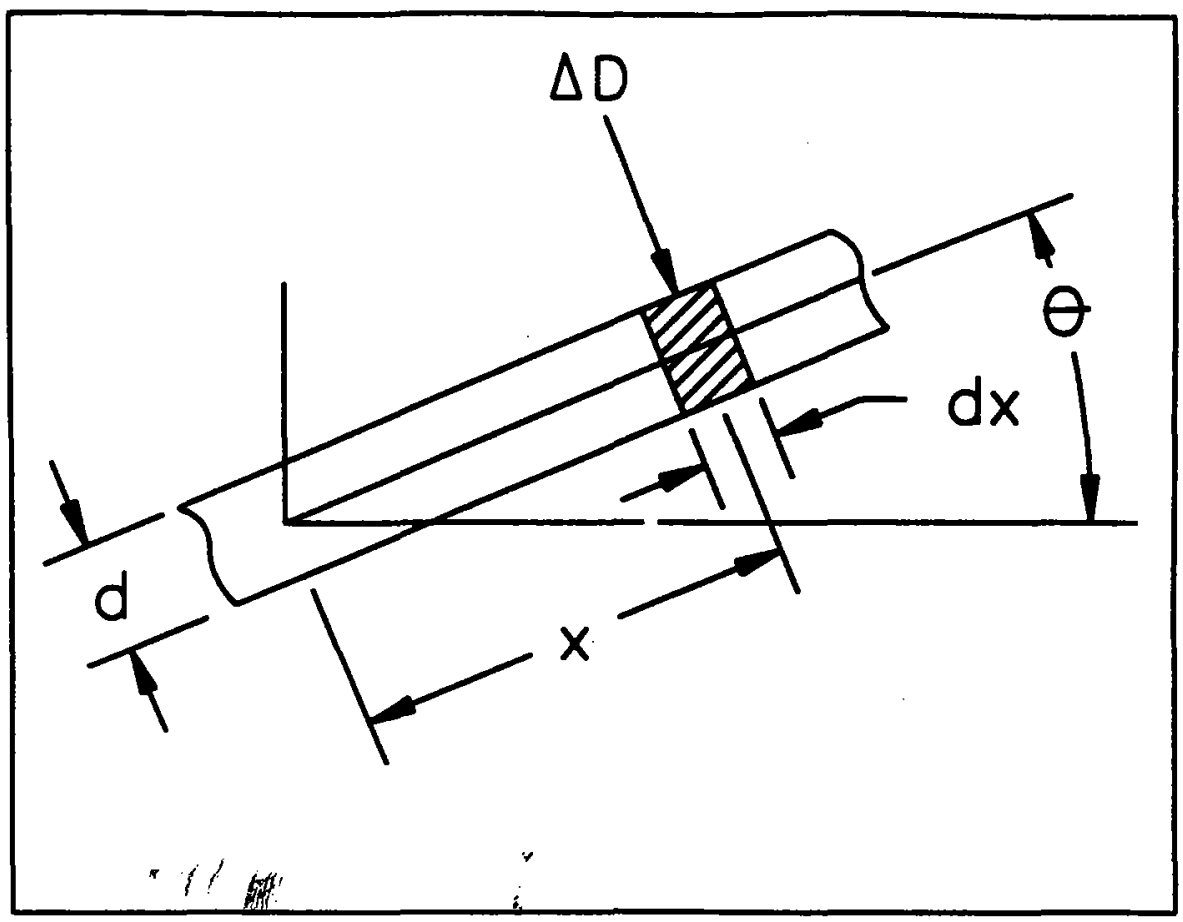

Figure 3-3: Strip Theory Formulation for Pitch Damping

As the angle of attack is varied, the moment arm for the drag force is $d \cos \alpha$.

The lift force is assumed to act at the same z-coordinate as the drag force, but at a distance $\frac{l}{4}$ from the nose where $l$ is the total length of the vehicle. This is an assumption based on the fact that the location of the lift force for a flat plate is at the quarter chord point. The lift force moment arm as a function of pitch angle is $\frac{l}{4} \cos \alpha-d \sin \alpha$. The assumptions made here are consistent with those made in chapter 2.

A third contribution to the viscous pitch moment is a pitch damping term of the form $M_{q|q|} q|q|$. Since this moment was not studied experimentally, an estimate can be made using a type of strip theory. Consider an area element $\Delta A$ on a slender cylindrical body at a distance $x$ from the center of rotation as in figure 3-3. The drag force on this element can be written as

$$
\Delta D=\frac{1}{2} \rho C_{D} \Delta A v|v|
$$

where $C_{D}=1.2$ is the drag coefficient for a cylinder, $v=x \dot{\theta}$ is the local transverse 
velocity. We can also write $\Delta A=d \cdot d x$ where $d$ is the cylinder diameter. The drag force is then

$$
\Delta D=\frac{1}{2} \rho C_{D} d(\dot{x} \dot{\theta})|(x \dot{\theta})| d x
$$

The moment about the origin from any element is $\Delta M=\Delta D \cdot x$ and the total moment is the integral over the length

$$
M=-2 \int_{0}^{\frac{l}{2}} \Delta D x d x=-\frac{\rho C_{D} d l^{4} \dot{\theta}|\dot{\theta}|}{64}
$$

If the effects of the three pods of the ABE vehicle are assumed to obey a law of superposition, the pitch damping coefficient can be calculated for each pod and summed. The result is

$$
M_{q|q|}=-255 \mathrm{~kg}-\mathrm{m}^{2}
$$

Note that this is a crude approximation because the ABE pods are not very long and slender. However, this method will suffice until full scale parameter identification can be used to measure the actual value.

When all of the pitch moments are summed the resulting moment equation is

$$
M_{\text {viscous }}=D(d \cos \alpha)+L\left(\frac{l}{4} \cos \alpha-d \sin \alpha\right)+M_{q|q|}|q| q \mid
$$

\subsubsection{Thruster Model}

The ABE vehicle will have five thrusters with which to maneuver in the longitudinal plane. Figure 3-4 shows their locations. There will be three thrusters for forward/reverse maneuvers and two for vertical motions. T1 and T2 are the thrusters behind the buoyancy pods and T3 is the thruster above and behind the electronics enclosure. T4 and T5 are the vertical thrusters. In figure 3-4, the arrows indicate directions of positive thrust.

A good model of the thruster dynamics is required for a realistic vehicle simulation since thrust is not delivered immediately upon command. A simplified thruster model 


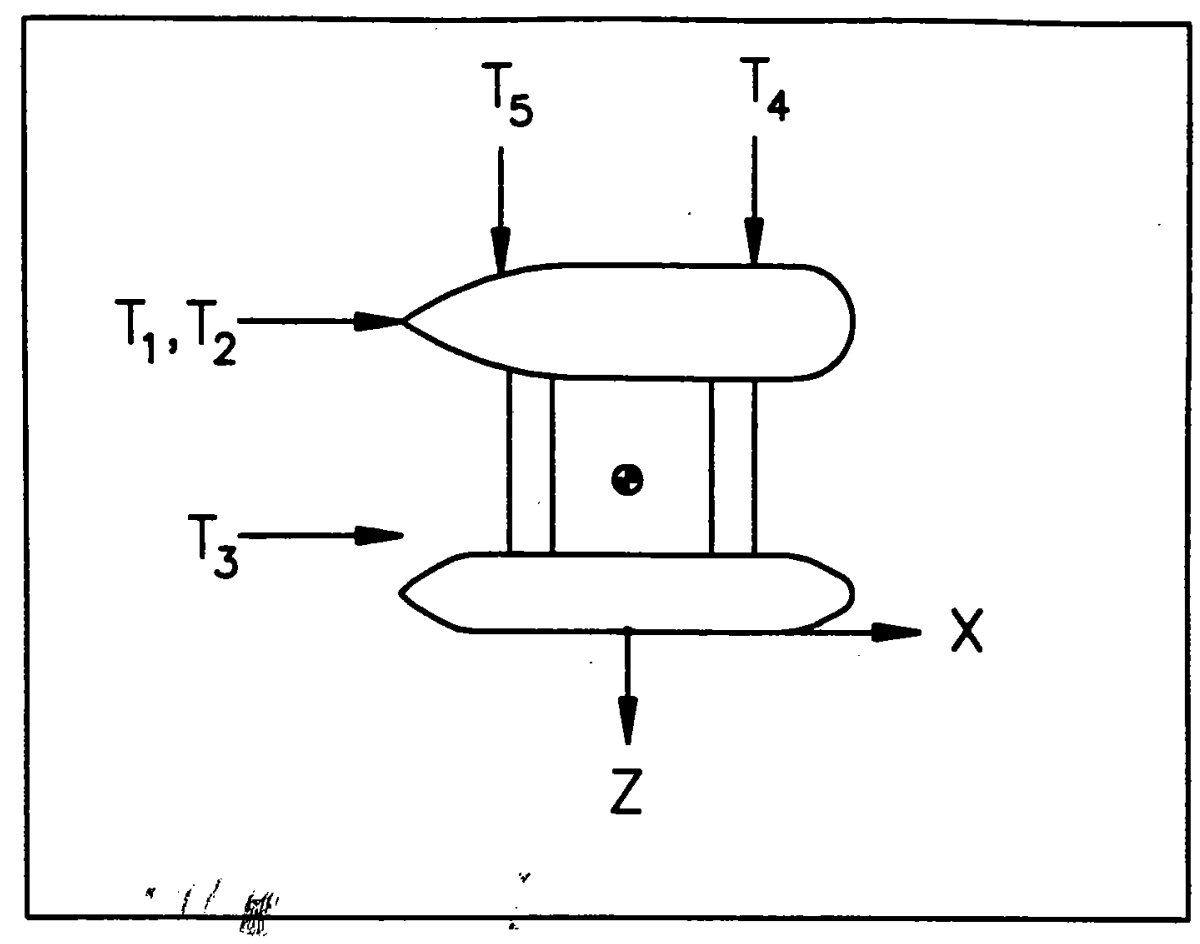

Figure 3-4: ABE Thruster Locations

developed by Woodford [14] is used.

The actual thrust is computed by integrating the angular velocity of each propeller $\omega$ according to the equation

$$
\dot{\omega}=\alpha\left(\frac{T_{d}}{C_{T}}-\omega|\omega|\right)
$$

where $T_{d}$ is the desired thrust. Then, the actual thrust is

$$
T_{a}=C_{T} \omega|\omega|
$$

The constants $\alpha=0.10$ and $C_{T}=0.066$ were determined experimentally for the ABE design thrusters in [14].

Figure 3-5 shows the step response of an ABE design thruster for three different step values. Note that the rise time depends on the amplitude of the commanded step. This property becomes very important for low thrust maneuvers such as hovering. The variable dynamic nature of the thrusters is well captured in the above model.

If $T_{i}$ denotes the thrust of thruster $i$, the thrust forces and moments can be written 


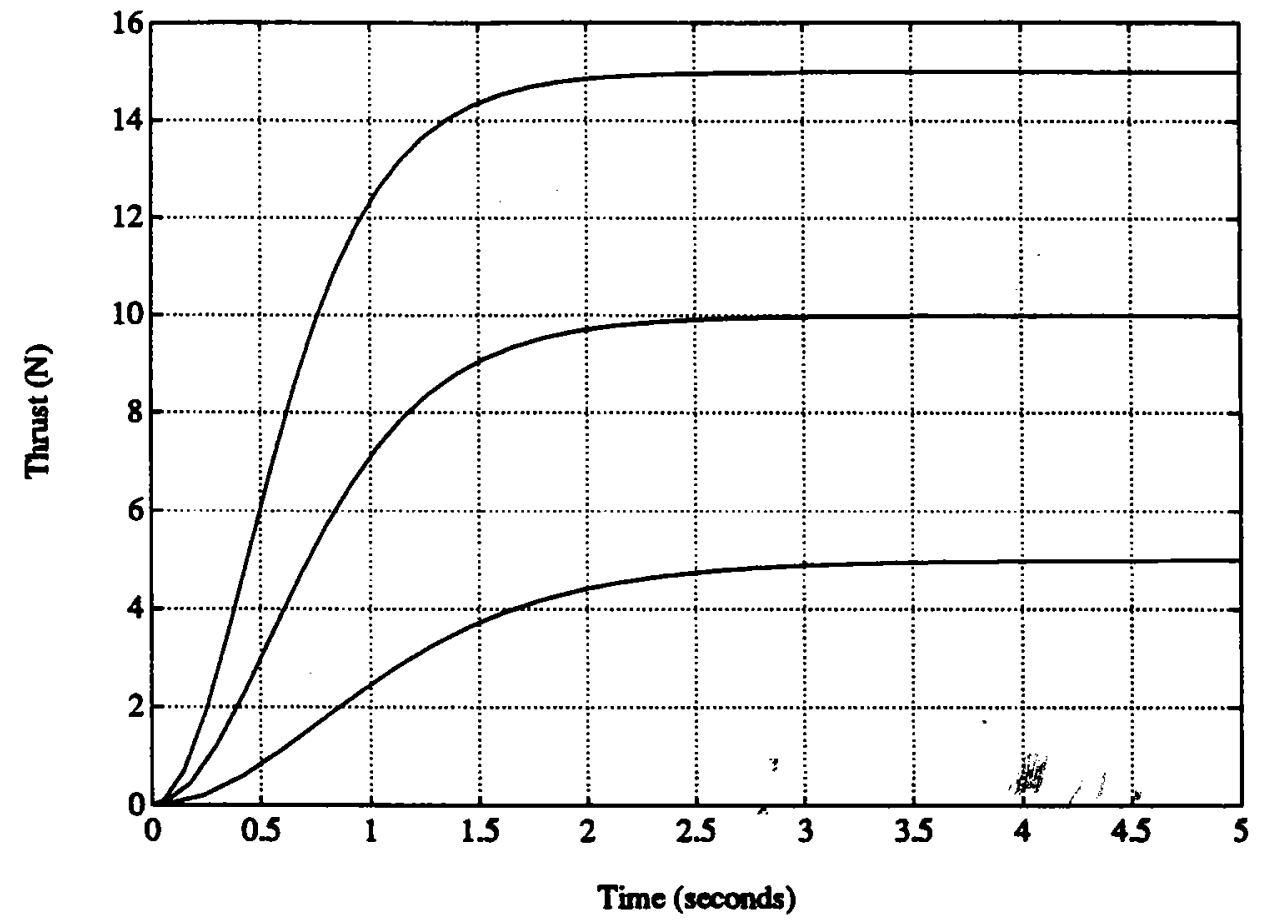

Figure 3-5: Thruster Step Responses 
as

$$
\begin{gathered}
X_{\text {thrust }}=T_{1}+T_{2}+T_{3} \\
Z_{\text {thrust }}=T_{4}+T_{5} \\
M_{\text {thrust }}=z_{T 1} T_{1}+z_{T 2} T_{2}+z_{T 3} T_{3}-x_{T 4} T_{4}-x_{T 5} T_{5}
\end{gathered}
$$

where the $z_{T i}$ and $x_{T i}$ are moment arms to the thrusters.

\subsubsection{Unmodelled Forces}

There are other forces acting on the vehicle that could be included in a more detailed model. For simplicity of computation and simulation, the following effects are neglected:<smiles>[AlH]</smiles>

- Forces on the vehicle due to interaction with the free surface. The vehicle is assumed to be deeply submerged.

- Forces due to vortex shedding. Cross flow drag, however, is accounted for by including the experimental results for nonzero angle of attack.

- Forces on the supporting structure. Fluid inertia and damping effects due to the struts are neglected.

- Forces due to external hardware. The ABE vehicle will have external attachments such as lights, cameras and sensors. The effects of these items will be included later as the vehicle design evolves.

- Linear component of the drag force. At low velocities the drag force has a linear nature. The drag coefficient could not be measured at very low speeds so this effect is not included.

\subsection{Suminary of Equations of Motion}

For simulation purposes, it is useful to write the equations of motion in the form $\dot{\vec{x}}=f(\vec{x}, \vec{u})$ where $\vec{x}$ is the vector of state variables and $\vec{u}$ is the vector of inputs. All 
Table 3.1: Summary of Vehicle Parameters

\begin{tabular}{|c|c|c|}
\hline$m=254.8 \mathrm{~kg}$ & $W=2500 \mathrm{~N}$ & $B=2500 \mathrm{~N}$ \\
\hline$I_{y}=237 \mathrm{~kg}-\mathrm{m}^{2}$ & $z_{g}=-.60 \mathrm{~m}$ & $z_{b}=-1.02 \mathrm{~m}$ \\
\hline$l=1.83 \mathrm{~m}$ & $d=0.90 \mathrm{~m}$ & $x_{g}=0 \mathrm{~m}$ \\
\hline$x_{b}=0 \mathrm{~m}$ & $z_{T 1}=-1.22 \mathrm{~m}$ & $z_{T 2}=-1.22 \mathrm{~m}$ \\
\hline$z_{T 3}=-0.38 \mathrm{~m}$ & $x_{T 4}=0.50 \mathrm{~m}$ & $x_{T b}=-0.50 \mathrm{~m}$ \\
\hline$X_{\dot{i}}=-38 \mathrm{~kg}$ & $X_{\dot{q}}=38 \mathrm{~kg}-\mathrm{m}$ & $Z_{\dot{b}}=-454 \mathrm{~kg}$ \\
\hline$M_{\dot{q}}=-997 \mathrm{~kg}-\mathrm{m}^{2}$ & $M_{q q q}=-255 \mathrm{~kg}-\mathrm{m}^{2}$ & $z_{\mathrm{q}}=-1.02 \mathrm{~m}$ \\
\hline$A_{\varepsilon}=.57 \mathrm{~m}^{2}$ & $A_{z}=2.0 \mathrm{~m}^{2}$ & \\
\hline
\end{tabular}

the terms which multiply accelerations are put into an inertia matrix $I$. Taking into account the simplifications made before, we have

$$
\begin{aligned}
& {\left[\begin{array}{ccc}
\left(m-X_{\dot{u}}\right) & 0 & \left(m z_{g}-X_{\dot{q}}\right) \\
0 & \left(m-Z_{\dot{w}}\right) & -m x_{g} \\
\left(m z_{g}-X_{\dot{q}}\right) & -m x_{g} & \left(I_{y}-M_{\dot{q}}\right)
\end{array}\right]\left(\begin{array}{c}
\dot{u} \\
\dot{w} \\
\dot{q}
\end{array}\right)=\left(\begin{array}{c}
X^{\prime} \\
Z^{\prime} \\
M^{\prime}
\end{array}\right)} \\
& X^{\prime}=-m w q+m x_{g} q^{2}-(W-B) \sin \theta+Z_{w} w q-D \cos \alpha+L \sin \alpha+T_{1}+T_{2}+T_{3} \\
& Z^{\prime}=m u q+m z_{g} q^{2}+(W-B) \cos \theta-X_{\dot{u}} u q-X_{\dot{q}} q^{2}-D \cdot{ }^{2} \pi \alpha-L \cos \alpha+T_{4}+T_{5} \\
& M^{\prime}=-m z_{g} w q-m x_{g} u q-\left(x_{g} W-x_{b} B\right) \cos \theta-\left(z_{g} W-z_{b} B\right) \sin \theta+X_{q} w q \\
& -\left(Z_{\mathfrak{w}}-X_{\dot{u}}\right) u w+D(d \cos \alpha)+L\left(\frac{l}{4} \cos \alpha-d \sin \alpha\right)+M_{q|q| q|q|} \mid \\
& +z_{T 1} T_{1}+z_{T 2} T_{2}+z_{T 3} T_{3}-x_{T 4} T_{4}-x_{T 5} T_{5}
\end{aligned}
$$




\section{Chapter 4}

\section{Simulation of the ABE}

\subsection{Introduction}

In this chapter, the details of the ABE computer simulation are discussed. Some basic diagnostics are presented which, in turn, illustrate some simulation pitfalls.

The ABE dynamics were simulated for different trajectories and control action using MathWorks $\subset M A T L A B^{T M}$ software. The equations of motion were integrated using a fourth order Runge-Kutta routine. In all, there are fifteen states that need to be integrated: three vehicle states $(u, w$ and $q)$, three inertial displacements $(X, Z$ and $\theta$ ), five thruster states (propeller velocities), two power states and two controller states.

\subsection{Power States}

The goal of this work is to control the ABE so that power is used in the most efficient manner possible. The most important power consumed is that used in actuating the thrusters. Power drawn by the ABE computer and sensors is assumed to be relatively constant whereas the thruster power requirement depends on how they are controlled.

The power drawn by each thruster is calculated using the experimental results in [14] for the ABE design thrusters. A linear relationship is used to calculate the input power in terms of the thrust delivered. Since we are only concerned with thruster 
power, a zero offset is used so that no thrust delivered means no power drawn.

The thruster power consumption formulae are

$$
\begin{gathered}
\text { Thrust }>0, \text { Power }=2.044 \cdot \text { Thrust } \\
\text { Thrust }<0, \text { Power }=-2.998 \cdot \text { Thrust }
\end{gathered}
$$

The thrust is in newtons and the power is in watts. Note that the power consumed is always positive. These equations are used to calculate the power up to thruster saturation. The thruster power is assumed to be independent of vehicle speed. This is an approximation based on static measurements by [14]. This estimate is assumed to be valid for the low speed ABE maneuvers.

The ABE thrusters are capable of delivering large thrust but are limited by the power available. For simulation, the thrusters are assumed to saturate at plus or minus 11 newtons. This keeps the power drawn near the 100 watt design limit for cruising conditions (1 knot).

The instantaneous input power is the sum of the power input to all of the thrusters. The output power is the sum of the absolute values of the mechanical power in each direction. The output power is

$$
P_{\text {out }}=\left|X_{\text {thrust }} u\right|+\left|Z_{\text {thrutt }} w\right|+\left|M_{\text {thrubt }} q\right|
$$

The input and output power are integrated for each simulation. These values can be compared to show the efficiency of a particular trajectory and control action.

\subsection{Diagnostics}

Several diagnostics were done on the simulation to verify its results. First, the responses to initial conditions were checked. Figure 4-1 shows the vehicle response to an initial pitch angle of 10 degrees. The figure shows the time history of the velocities $u, w$, and $q$, and the inertial states $X, Z$ and $\theta$. 

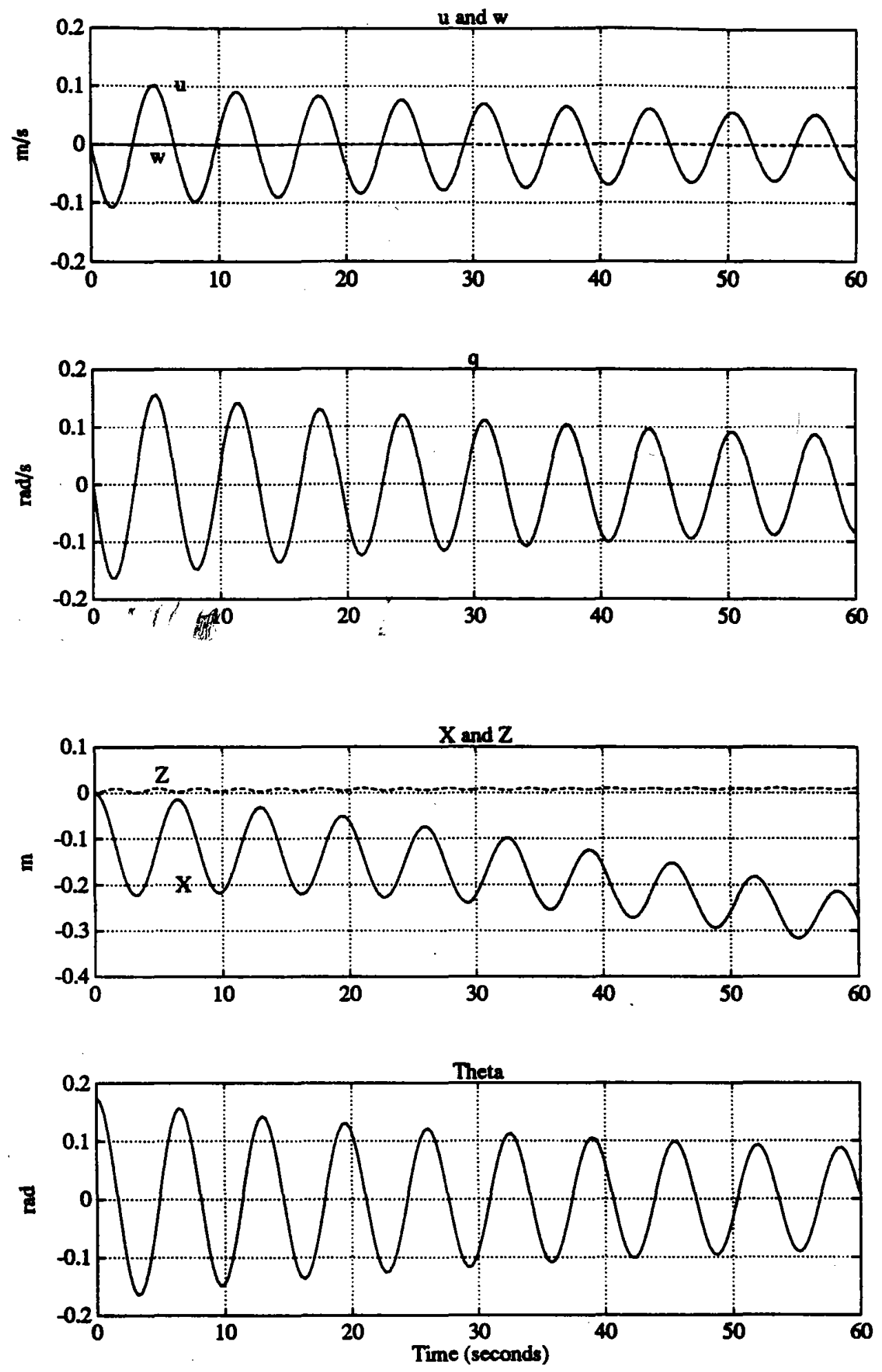

Figure 4-1: Response to 10 Degree Initial Pitch Angle 
The most notable thing about figure 4-1 is the pendulum mode which dominates the dynamics. This is the fundamental pitch response which has a period near 6.4 seconds which corresponds to a frequency of about .16 Hertz. This oscillation appears in all of the vehicle states since the origin of the body coordinate system is not at the center of gravity.

The pendulum mode becomes important when one wants to control the inertial coordinates $X$ and $Z$. The pitch of the vehicle will not be controlled except in a quasistatic manner. This means that the pendulum mode will be tolerated and control action should not be used to try to counter it. This requires that the control action work on the tracking error in terms of the position of the center of gravity, not the position of the body coordinate system origin.

Figure 4-1 also shows the slow decay rate of the pitch response. The vehicle is still pitching to 5 degrees (.09 radians) after 60 seconds. This large of a response is unlikely and is probably due to too small estimates of $M_{\dot{q}}$ and $M_{q|q|}$. There is a strong desire to tweek these parameters so that the response is more reasonable. However, it will be resisted until experiments or full scale identification can be used to determine these parameters. Underestimating these two parameters should not greatly affect the problem of controlling the $X$ and $Z$ coordinates if the center of gravity is controlled.

Figure 4-1 also shows a small drift in the $X$ coordinate of the vehicle. The vehicle moves $25 \mathrm{~cm}$ backward in 60 seconds. This is an artifact of thow simulation was started. If the initial condition had been a negative pitch angle, the vehicle would creep forward.

A good example of how drag dominates the vehicle dynamics is shown in figure 4-2. The vehicle response is given for initial conditions on both velocities $u$ and $w$. The initial conditions of $u(0)=.50 \mathrm{~m} / \mathrm{s}$ and $w(0)=.25 \mathrm{~m} / \mathrm{s}$ correspond to an angle of attack of 26 degrees. There is both lift and drag on the vehicle.

It is apparent that the drag coefficients are not the same for the forward and vertical directions. The two responses have correspondingly different decay rates. As the velocities decay, the angle of attack increases. This results in an increase in the drag and lift coefficients which then produces faster decay rates. 

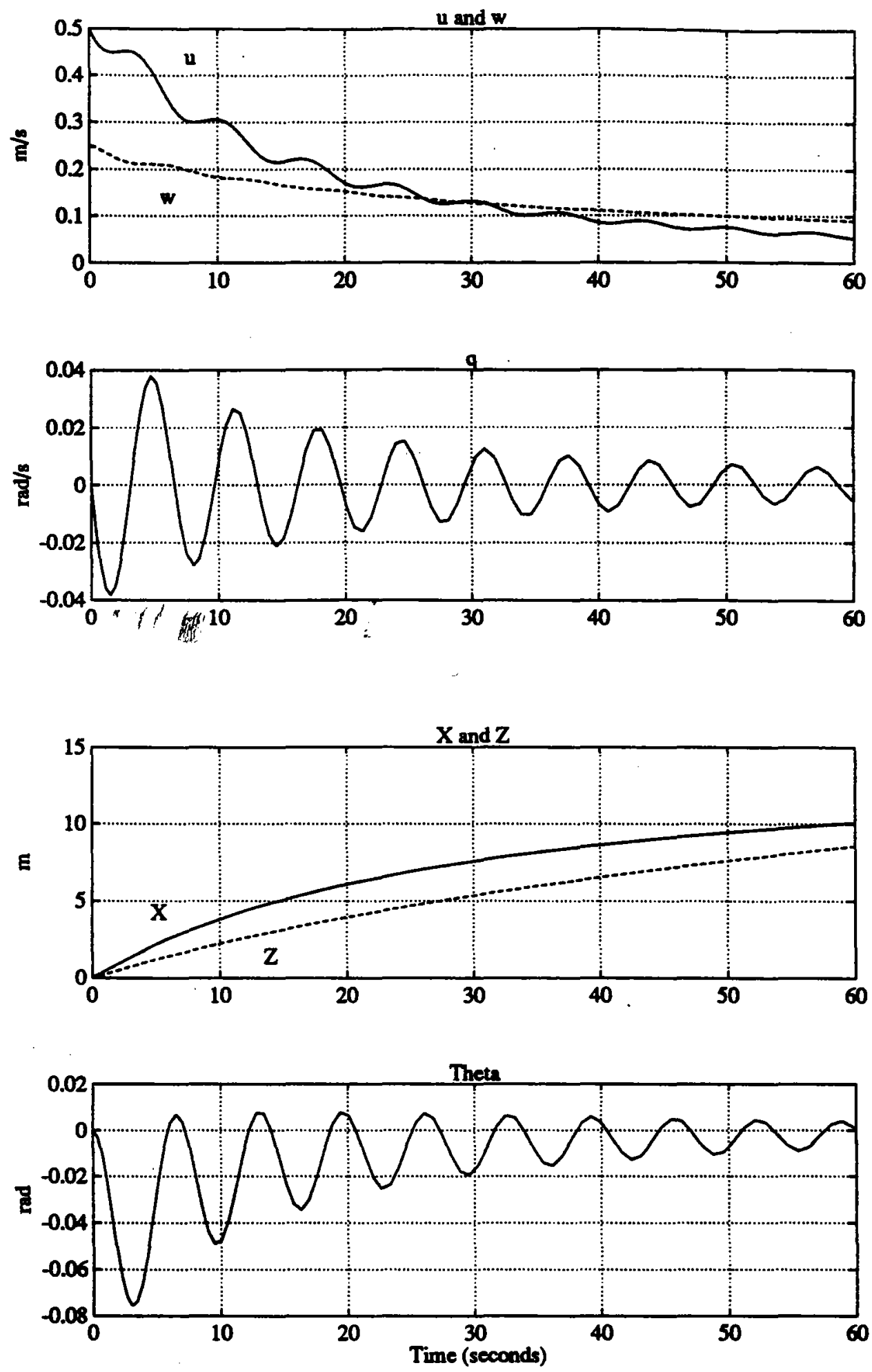

Figure 4-2: Response to Initial Conditions: $\mathrm{u}(0)=.50 \mathrm{~m} / \mathrm{s}, w(0)=.25 \mathrm{~m} / \mathrm{s}$ 


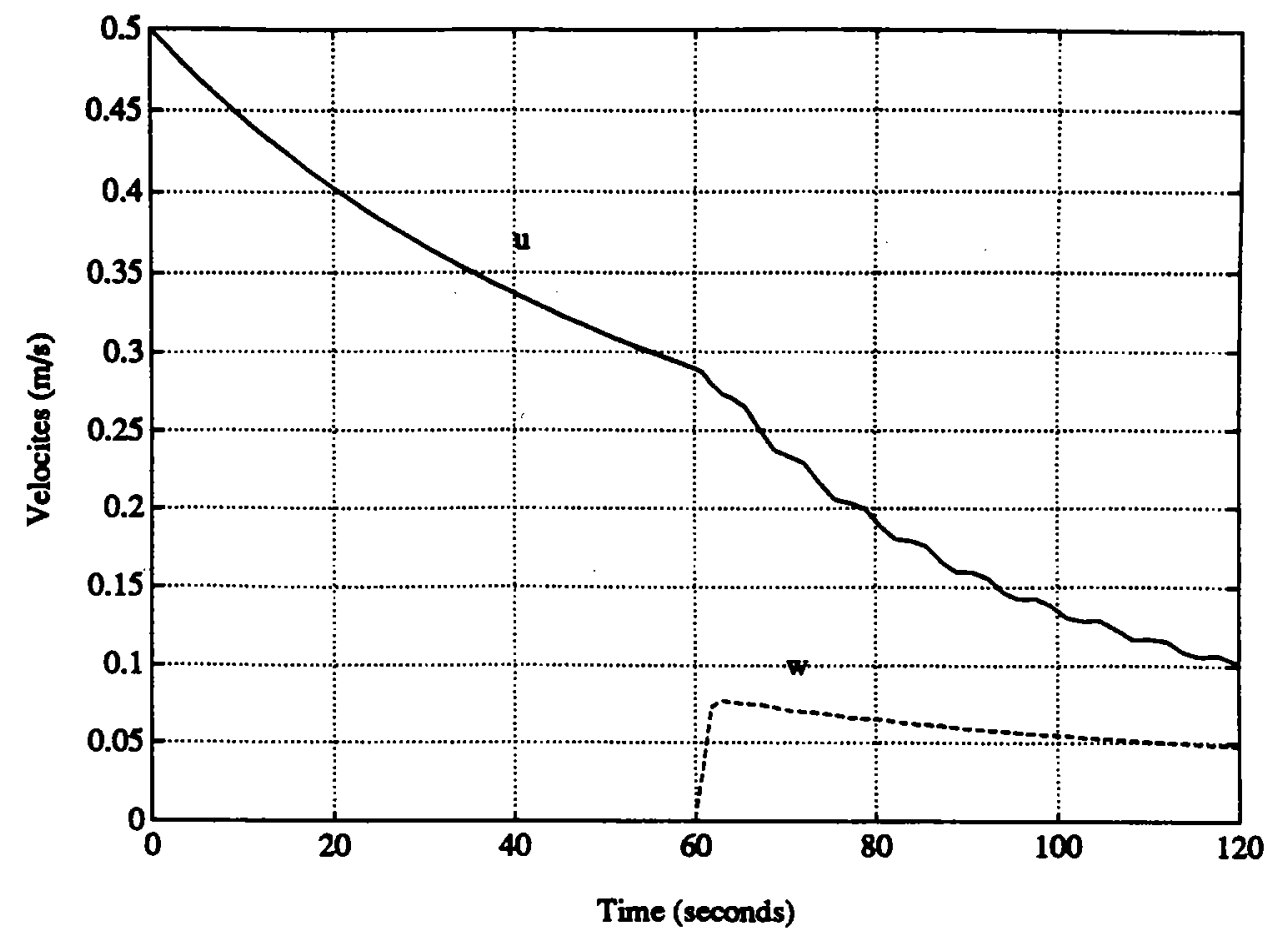

Figure 4-3: Initial Condition Response with a Disturbance

Consider the case where there is only an initial condition on $u$. For the same initial velocity, the decay would be much slower. In the case shown in figure 4-2, u decays to 20 percent of its initial value in 35 seconds. If thereswere no vertical velocity present, this decay would take 300 seconds.

A simple test was done to verify that the simulation shows correct drag behavior. An initial condition was given only to $u$. Then, the decay was interrupted by an impulse-like disturbance in the vertical direction at 60 seconds. Figure 4-3 shows the result. A vertical velocity $w$ developed with a corresponding increase in the $u$ decay rate. This shows how the surge decay rate depends on the existence of a vertical disturbance.

Figures 4-2 and 4-3 also demonstrate the coupling that is present between surge, heave and pitch. Even for no initial conditions on the pitch angle, oscillatory responses up to 4 degrees were induced by the other motions. The effects of pitch oscillation 
are also apparent in the surge and heave velocities.

The second type of diagnostic test done was to give each thruster a step command. A $10 \mathrm{~N}$ step was used which is near the saturation level. The corresponding thruster response time is around two seconds. Figure 4-4 shows the vehicle response for a step command on the lower forward thruster.

The forward velocity reaches a steady value near 2 knots in 60 seconds. This slow response time is due to thruster dynamics and the nonlinear nature of the drag force. There is a strong pendulum motion induced since the thruster does not act at the center of the hydrodynamic force. The pitch angle oscillates about a small positive value (nose up) which is the correct behavior. The vertical response is negligible.

Figure $4-5$ is a vertical thruster diagnostic test. The aft vertical thruster was given a step commangl of $10 \mathrm{~N}$. The response is slightly faster than in the forward case because the drag coefficient is larger. This drag force has a faster rise time which makes the vehicle reach its terminal velocity faster. Figure 4-5 shows a similar positive pitch response which is correct for positive aft thrust.

The simple diagnostics presented here verify that the simulation is giving reasonable vehicle behavior. They demonstrate the basic drag and thrust characteristics of the $A B E$ in addition to the well observed pendulum mode. Basic behavior in simple tests like this give new insights which can be incorporated into the ABE mechanical design.

\subsection{Problems with Simulation}

In the simulation world it is easy to lose the physics of the process that we are trying to simulate. Oftentimes, simulation is too perfect. There are no disturbances except those imposed by the investigator. There is no sensor noise or feedback delays. It is important that simulation results are critically examined. Changing parameters to better match reality without understanding the physics involved can have disastrous results. This sort of tampering will not be used since this simulation is a first iteration in the study of the ABE problem. Full scale system identification will be used later 

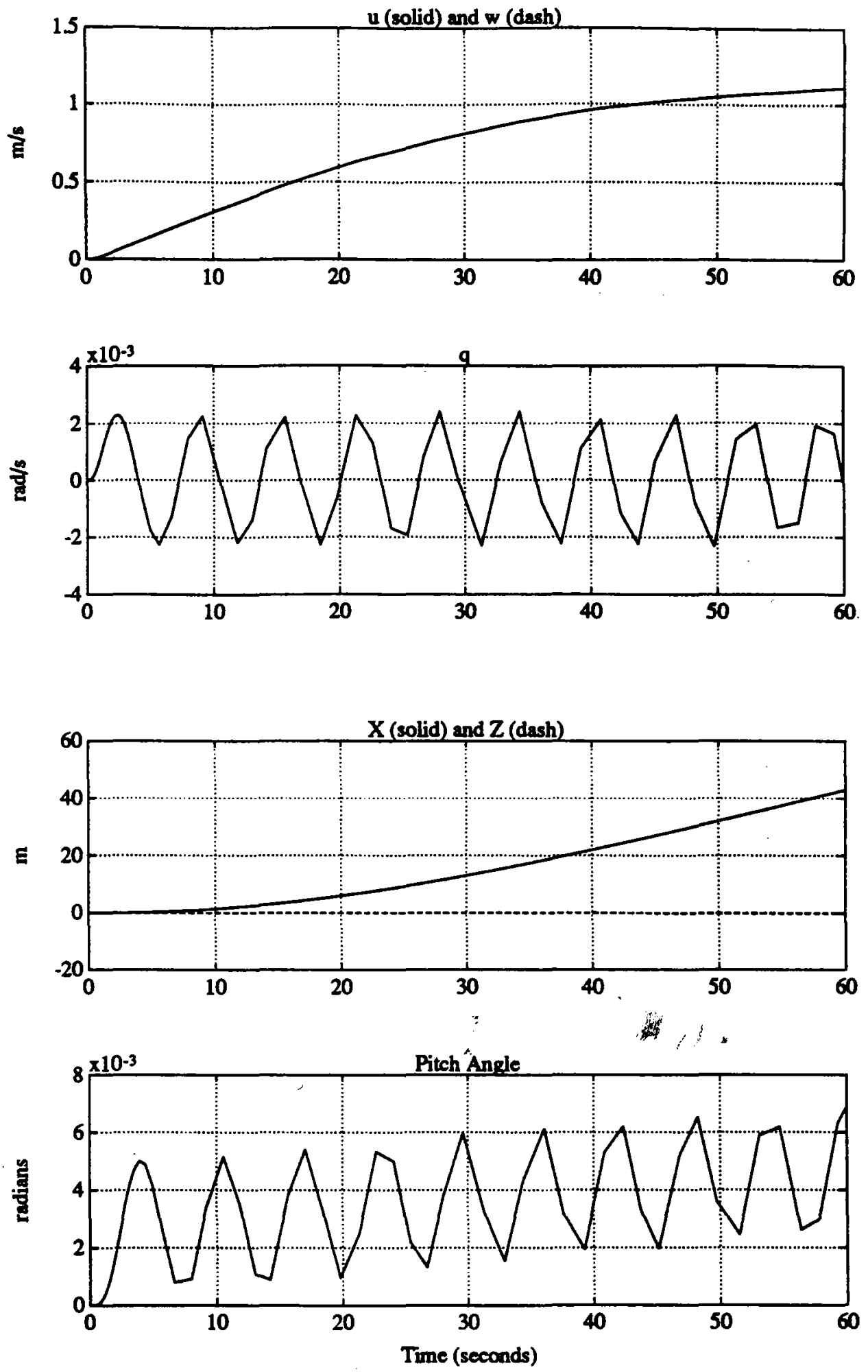

Figure 4-4: Response to $10 \mathrm{~N}$ Step Command on Main Forward Thruster 

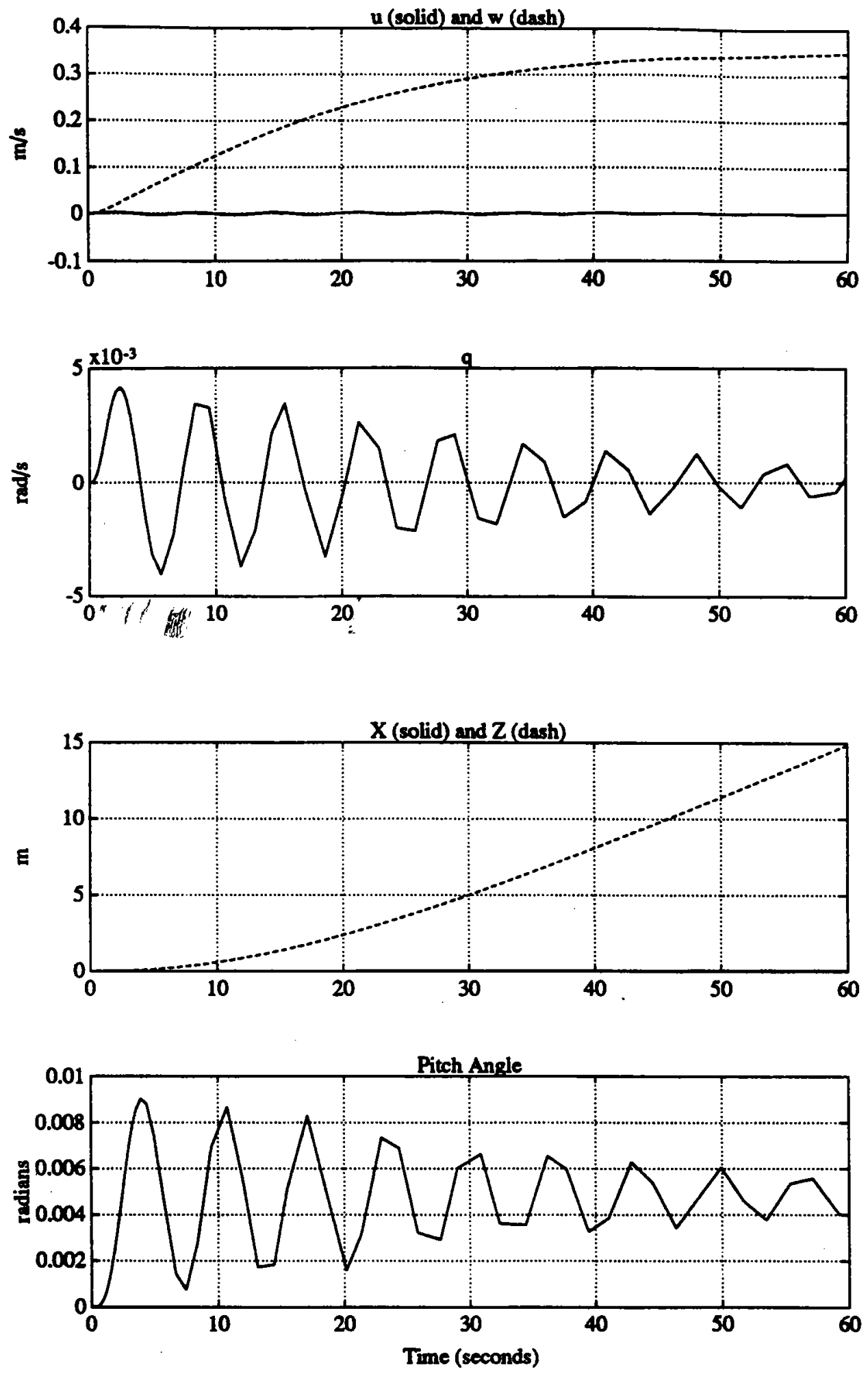

Figure 4-5: Response to a $10 \mathrm{~N}$ Step Command on Aft Vertical Thruster 
to fine tune the simulation model.

One significant problem with the ABE simulation is that the viscous forces on the vehicle are known only up to an angle of attack of 45 degrees. Whatever happens after that is speculation. It would not be accurate to extrapolate the linear curves on the drag and lift coefficients because this would result in large errors near 90 degrees.

A model such as those suggested in chapter 2 could be used to estimate the forces in the gray area between 45 and 90 degrees. The problem with doing this is that the ABE vehicle has the properties of both a streamlined body and a bluff body. A model would have to be formulated which incorporates the drag nature of a bluff body and the lift nature of a streamlined body. The current solution for the ABE simulation is to limit the angle of attack to less than 45 degrees. A general model may be developed later for more challenging trajectories.

Another problem is that the model viscous forces are for steady state. The transient nature of the drag force could not be measured in the towing tank. Therefore, the linear component of the drag force for low velocities is not modelled. It takes a very long time for drag alone to stop the vehicle with the drag model used in simulation. This is another instance where there is a desire to tweek the parameter so that the response seems more physical.

One solution is to introduce a linear drag term which gives the system a maximum time constant of $\mathbf{3 0}$ seconds for example. This is a dumb 4 pproach which does not attempt to understand the actual physics. Simulation quick fixes like this are not necessary for the ABE study since we are not concerned with very low speed maneuvers. The experimental drag results are valid in the regime in which the ABE will operate. The linear drag component will be determined experimentally when the full scale vehicle is available.

In this chapter some basic simulation behaviors were presented which illustrate some of the difficulties in mimicking reality. The simple diagnostics show that the model behavior is correct to first order. The following chapters include simulation for typical ABE trajectories with two different control methodologies. 


\section{Chapter 5}

\section{Controller Design}

\subsection{Introdúgtion}

In appendix $A$, the equations of motion are rewritten to have the form

$$
\left[\begin{array}{c}
\ddot{X}_{\bullet} \\
\ddot{Z}_{0}
\end{array}\right]=\vec{f}(u, w, q, \theta)+B\left[\begin{array}{l}
X_{\text {thruot }} \\
Z_{\text {thruot }}
\end{array}\right]
$$

where $\vec{f}$ is a nonlinear vector function of the state and $\boldsymbol{X}_{\text {thruot }}$ and $Z_{\text {thruot }}$ are the net thrusts in the the $x$ and $z$ directions respectively. The equations are written such that $X_{0}$ and $Z_{0}$ are the control variables of interest. This is a natural way to set up the ABE control problem since ABE's primary mission will be to track a prescribed trajectory. The pitch angle is not controlled here since the movable weight will be operating in a quasistatic manner. The effects of pitch control can be included later as a disturbance.

The simple transformation described in appendix A can be used to control the center of gravity coordinates $X_{c g}$ and $Z_{c g}$. This will prove useful in keeping the pendulum mode of the vehicle out of the controller. This is practical since the ABE will be well instrumented in pitch.

The model used for controller design does not include the thruster dynamics that are in the simulation model. They are not included because feedback control would 
require a velocity sensor on each propeller which is not very practical. The possibility of thruster saturation is also not included in the model for the controller. These two effects will test the robustness of the controllers to unmodelled dynamics.

Two controllers are developed in this chapter: a proportional plus derivative controller with nonlinear feedforward and a sliding mode controller. The theory associated with each controller is briefly summarized. The performance of the controllers over different vehicle trajectories will be discussed in the next chapter.

\subsection{Proportional Plus Derivative (PD) Control}

PD control is a simple but effective method of control. The tracking error and its derivative are fed back with gains selected to place the error dynamics. Although PD control is usually a linear control technique, it can be applied to nonlinear systems. A feedforward term is used to effectively cancel the nonlinear dynamics.

Given the above form of the equations of motion, a tracking error can be defined as

$$
\overrightarrow{\vec{x}}=\vec{x}-\vec{x}_{d}
$$

where $\vec{x}$ is the control variable of interest with elements $X_{0}$ and $Z_{0}$ or $X_{c g}$ and $Z_{c g}$.

The following control law will give exact tracking if there are no model uncertainties

$$
\overrightarrow{T H}=-\hat{B}^{-1}\left(\overrightarrow{\hat{f}}-\vec{K}_{D} \dot{\overrightarrow{\tilde{x}}}-\vec{K}_{P} \overrightarrow{\tilde{x}}\right)
$$

The caret notation denotes a best estimate of a quantity.

The gains $\vec{K}_{D}$ and $\vec{K}_{P}$ are chosen so that the dynamics are stable with good performance. The gains can be tuned for each direction independently. The feedforward term $\hat{B}^{-1} \overrightarrow{\hat{f}}$ cancels the nonlinearity so that the selection of gains is a simple placement of poles. The dynamics to be placed are 


$$
\ddot{x}+K_{D} \dot{x}+K_{P} x
$$

for each direction. For a controller bandwidth of $\lambda$ and a damping ratio of $\zeta$, the desired gains are

$$
\begin{gathered}
K_{D}=2 \zeta \lambda \\
K_{P}=\lambda^{2}
\end{gathered}
$$

Although PD control is not a robust method that will actually be implemented, it is useful in simulaton. It gives good initial estimates of the thrusts required for trajectory control and provides a baseline with which other methods can be compared.

\subsection{Sliding Mode Control}

The sliding mode control methodology provides for robust control in the presence of structured uncertainties. The technique reduces the control of an n-dimensional problem to that of a first order stabilization of a sliding variable. Slotine [11] gives a good overview of the theory and design method. A brief summary of the basic method is given here as it applies to the control of the two input/two output ABE system.

A tracking error is defined in the same manner as for the PD control. A measure of the tracking error is the sliding variable $\overrightarrow{3}$. Since the $\vec{x}$ dynamics are second order, the sliding variable components are given by

$$
s_{i}=\left(\frac{d}{d t}+\lambda\right) \overline{x_{i}}
$$

where $\lambda$ is the strictly positive constant bandwidth. The sliding variable can be thought of as defining a sliding surface in the phase plane of the dynamics. 
If the initial state $\vec{x}(0)$ is equal to the desired initial state, it can be shown that $\vec{s} \equiv \overrightarrow{0}$ is a linear differential equation which has the solution $\overrightarrow{\tilde{x}} \equiv \overrightarrow{0}$. This is the condition for perfect tracking.

To keep the sliding variable at zero, a sliding condition is required to keep the $\overrightarrow{\vec{b}}$ dynamics tending towards $\overrightarrow{0}$. The condition is

$$
\frac{1}{2} \frac{d}{d t} s_{i}^{2} \leq-\eta_{i}\left|s_{i}\right|
$$

where $\eta_{i}$ is a positive constant. Whenever the sliding variable moves away from zero, the sliding condition guarantees that it moves back. The equation $\dot{\vec{s}}=0$ can be solved for an equivalent thrust vector $\overrightarrow{T H}$ that would give perfect tracking if the model is known exactly. For the above form of the equations of motion, the equivalent control is

$$
\overrightarrow{T \vec{H}}=\hat{B}^{-1}\left(\ddot{\vec{x}}_{d}-\lambda \dot{\overrightarrow{\tilde{x}}}-\overrightarrow{\hat{f}}\right)
$$

A switching action is added to the equivalent control to counter against discrepancies in the model. The switching term acts like a PD term since it adds control action which depends on the sign of the tracking error. The new control law is

$$
\overrightarrow{T H}=\hat{B}^{-1}\left(\dot{\overrightarrow{x_{\tau}}}-\overrightarrow{\hat{f}}-\vec{k} \operatorname{sgn}(\vec{s})\right)
$$

where $\dot{\overrightarrow{x_{\mathrm{r}}}}=\ddot{\overrightarrow{x_{d}}}-\lambda \dot{\overrightarrow{\vec{x}}}$ is a notational simplification. The vector $\vec{k} \operatorname{sgn}(\vec{s})$ has elements $k_{i} \operatorname{sgn}\left(s_{i}\right)$ where $\vec{k}$ is a gain vector which is chosen large enough to counter disturbances and parametric errors in the model. sgn refers to the sign function which takes the sign of its argument.

For the sliding controller to be robust to structured parametric uncertainties, the gain vector must be selected sufficiently large. First, bounds must be set on the dynamics. The uncertainties in the vector function $\vec{f}$ will be considered to have an additive form and the matrix $B$ will have a multiplicative error. The bounds on these are defined as follows 
Table 5.1: Allowable Parameter Variations

\begin{tabular}{|c|c||c|c|}
\hline parameter & $\begin{array}{c}\text { percent } \\
\text { variation }\end{array}$ & parameter & $\begin{array}{c}\text { percent } \\
\text { variation }\end{array}$ \\
\hline \hline$m$ & 10 & $B$ & 10 \\
\hline$z_{g}$ & 10 & $x_{b}$ & 10 \\
\hline$z_{b}$ & 10 & $X_{\dot{u}}$ & 20 \\
\hline$X_{\dot{q}}$ & 20 & $Z_{\dot{b}}$ & 20 \\
\hline$M_{\dot{q}}$ & 20 & $I_{y}$ & 20 \\
\hline$A_{z}$ & 20 & $A_{z}$ & 20 \\
\hline$\rho$ & 10 & $d$ & 20 \\
\hline$l$ & 20 & $z_{T 1}$ & 10 \\
\hline$z_{T 2}$ & 10 & $z_{T 3}$ & 10 \\
\hline$M_{q|q|}$ & 50 & $x_{T 4}$ & 10 \\
\hline$x_{T b}$ & 10 & $\alpha$ & 20 \\
\hline$C_{T}$ & 20, & $x_{g}$ & 10 \\
\hline$C_{4} C_{D}$ & 20 & $C_{L}$ & 20 \\
\hline
\end{tabular}

$$
\begin{gathered}
\left|\hat{f}_{i}-f_{i}\right| \leq F_{i} \quad i=1,2 \\
B=(I+\Delta) \hat{B} \quad\left|\Delta_{i j}\right| \leq D_{i j} \quad i=1,2 \quad j=1,2
\end{gathered}
$$

The actual values of $F_{i}$ and $D_{i j}$ depend on the state variables and the maximum and minimum values of the model parameters. In this controller design, each parameter is allowed a percentage variation from a nominal value. Table 5.1 lists the uncertain parameters in the ABE model and their allowable variations.

Arbitrary variations are not allowed since the uncertainty must lie within the range space of the $B$ matrix, i.e., there is a controllability requirement. Also, the estimate $\hat{B}$ must be invertible and continuously dependent on parametric uncertainty.

The actual calculation of the bounds $F_{i}$ is quite complicated since the $f_{i}$ depend on the elements of the inverse of the inertia matrix and nonlinear combinations of the state variables and the uncertain parameters. These bounds are computed in simulation using simple logic to determine the maximum and minimum value of each 
term.

The method used to compute the $F_{i}$ is briefly summarized here. If the elements of $\vec{f}$ are written as

$$
f_{i}=\sum_{k=1}^{m} a_{k} Z_{k}
$$

where the $a_{k}$ are uncertain parameters or combinations of uncertain parameters, and the $Z_{k}$ are combinations of the state variables at a particular point in time. The difference between the estimate $\hat{f}_{i}$ and $f_{i}$ is

$$
\hat{f}_{i}-f_{i}=\sum_{k=1}^{m}\left(\hat{a}_{k}-a_{k}\right) Z_{k}
$$

and it is bounded by

$$
\left|\hat{f}_{i}-f_{i}\right| \leq \sum_{k=1}^{m}\left|\hat{a}_{k}-a_{k}\right|\left|Z_{k}\right|=F_{i}
$$

The problem is now how to compute $\left|\hat{a}_{k}-a_{k}\right|$. It can be shown with a simple geometric argument that the difference is bounded by $\left(a_{k \text { mas }}-a_{k \text { min }}\right)$ regardless of the sign of the maximum and minimum of $a_{k}$.

To compute the gain margin on the matrix $B$, a similar approach is used. First, the $B$ matrix is written as

$$
B_{i j}=\sum_{l=1}^{n} b_{l_{i j}} Z_{l_{i j}}
$$

where the $b_{l}$ are combinations of the uncertain parameters and $Z_{l}$ are expressions involving the state variables. From the previous definition of the multiplicative error $\Delta$, we can write

$$
\begin{gathered}
B=\hat{B}+\Delta \hat{B} \\
\Delta=(B-\hat{B}) \hat{B}^{-1}
\end{gathered}
$$


and then it follows after some linear algebra that

$$
\begin{gathered}
\left|\Delta_{i j}\right| \leq\left|(B-\hat{B})_{i i}\right|\left|\hat{B}_{i j}^{-1}\right|+\left|(B-\hat{B})_{i j}\right|\left|\hat{B}_{j j}^{-1}\right|=D_{i j} \\
\left|(B-\hat{B})_{i j}\right| \leq \sum_{l=1}^{n}\left|b_{l_{i j}}-\hat{b}_{l_{i j}}\right|\left|Z_{l_{i j}}\right|
\end{gathered}
$$

The absolute values can be calculated using the maximum and minimum values of the $b_{l}$ with the sign considerations used before.

Once the bounds are defined, the gain vector can be computed by solving a second order linear equation. The only unknown variables are the gains $k_{i}$. Slotine [11] presents the proof of the existence of a unique solution. The equations which guarantee that the sliding condition is met (i.e., robustness requirement) are:

$$
\left(1-D_{i i}\right) k_{i}+\sum_{j \neq i} D_{i j} k_{j}=F_{i}+\sum_{j=1}^{n} D_{i j}\left|\dot{x}_{r i}-\hat{f}_{j}\right|+\eta_{i} \quad i=1,2
$$

The control law can be further modified to improve tracking performance and power consumption. One problem with the current control law is that the sgn function is discontinuous. This causes the control command to chatter which consumes a lot of power and introduces high frequency excitation to the system.

This problem is easily solved by introducing a continuous approximation to the sgn function. The saturation function is defined as

$$
\begin{gathered}
\operatorname{sat}(x)=x \quad|x| \leq 1 \\
\operatorname{sat}(x)=\operatorname{sgn}(x) \quad|x|>1
\end{gathered}
$$

A boundary layer $\vec{\phi}=\left[\phi_{m} \phi_{z}\right]$ is defined around the sliding surface defined by $\vec{s}=\overrightarrow{0}$, inside of which, there is a continuous control law. This eliminates the chattering which happens when the sliding surface is crossed. The new control law is

$$
\overrightarrow{T H}=\hat{B}^{-1}\left(\dot{\vec{x}}_{r}-\overrightarrow{\hat{f}}-\overrightarrow{\vec{k}} \operatorname{sat}(\vec{s} / \vec{\phi})\right)
$$


where the vector $\vec{k} \operatorname{sat}(\vec{s} / \vec{\phi})$ has elements $k_{i} \operatorname{sat}\left(s_{i} / \phi_{i}\right)$.

The boundary layer is dynamically adjusted to best use the available bandwidth of the controller. The method used here is an extension of the single-input/aingleoutput method presented in [11]. There is a multi-input/multi-output example in [12].

First, a gain margin matrix is defined to have the elements

$$
\beta_{i j}=\left(\frac{B_{i j \max }}{B_{i j \min }}\right)^{\frac{1}{2}}
$$

The maximum and minimum elements of $B$ can be calculated using the previously computed bounds $D_{i j}$. The equations which govern the dynamics of each boundary layer can be summarized as

$$
\begin{gathered}
k\left(x_{d}\right)_{i}>\left(\beta_{d}^{-1} \lambda \vec{\phi}\right)_{i} \Rightarrow \dot{\phi}_{i}+\lambda \phi_{i}=\left(\beta_{d} \vec{k}\left(x_{d}\right)\right)_{i} \\
k\left(x_{d}\right)_{i} \leq\left(\beta_{d}^{-1} \lambda \vec{\phi}\right)_{i} \Rightarrow \dot{\phi}_{i}+\left(\left(\beta_{d}^{-1}\right)^{2} \lambda \vec{\phi}\right)_{i}=\left(\beta_{d}^{-1} \vec{k}\left(x_{d}\right)\right)_{i}
\end{gathered}
$$

$\beta_{d}$ denotes the gain margin matrix evaluated for the desired state. Similarly, $\vec{k}\left(x_{d}\right)$ is the gain vector for the desired state. The pseudo inverse matrix $\beta^{-1}$ is the matrix of reciprocals of the elements of $\beta$. The new gain vector is

$$
\vec{k}(x)=\vec{k}(x)-\vec{k}\left(x_{d}\right)+\beta_{d}^{-1} \lambda \vec{\phi}
$$

The required initial condition on the boundary layer is $\vec{\phi}(0)=\beta_{d} \vec{k}\left(x_{d}(0)\right) / \lambda$.

The implementation of a dynamic boundary layer adds two states to the simulation. Also, the amount of computation for determining the gain vector is roughly doubled since the gain must be computed for both the actual and the desired trajectory. The added complexity of sliding mode control pays off in performance and robustness to parameter uncertainty.

The sliding mode controller can be enhanced by adding adaptive behavior with 
on-line parameter estimation [11]. The adaptation laws can be formulated so that adaptation begins when the sliding variable exits the boundary layer. It would then turn off when good tracking resumes. This would ensure that the system would only adapt to actual model changes and not chase parameter drift.

In the following chapter, the two controllers developed here will be put through some typical ABE paces. The point is not to compare PD control and sliding mode control. Rather, the two controllers are compared in terms of how they handle the ABE control problem.

\section{ith}




\section{Chapter 6}

\section{Evaluation of Controller}

\section{Performance}

\subsection{Introduction}

Each of the controllers developed in chapter 5 is evaluated in terms of how it performs on three typical ABE trajectories. The goal here is not to design the actual ABE control system. Rather, the purpose is to explore the use of different control methodologies for the ABE control problem.

\subsection{Trajectories of Interest}

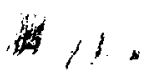

The primary ABE trajectory (hereafter referred to as track I) is forward level motion at the design cruising speed of one knot. The vehicle starts at rest, goes through a brief acceleration period, and then maintains the cruising speed. The acceleration period for simulation is chosen to be $\mathbf{1 0}$ seconds. This is a fast acceleration time which means that the thrusters may saturate to maintain the desired trajectory. The actual ABE ramp time can be adjusted to conserve power if the mission allows it.

The second trajectory (track II) is a straight vertical descent. Again, there is a short acceleration period followed by a cruising speed of $.25 \mathrm{~m} / \mathrm{s}\left(\sim \frac{1}{2}\right.$ knot). This maneuver would be used for getting close to something of interest and in the hitching 
process.

The last and most interesting trajectory (track III) is a combined forward/vertical motion. The trajectory is chosen so that the angle of attack is challenging. The distance traveled in $X$ is twice the distance traveled in $Z$ at any given time. The desired velocities are chosen 80 that in one minute, the vehicle has gone ten meters down and twenty meters forward. This track captures all of the longitudinal dynamics since it involves trajectories for $X, Z$ and potentially $\theta$.

\subsection{Performance}

The ABE dynamics were simulated for each trajectory with a PD controller and a sliding mode coritrolker as developed in chapter 5 . Both controllers were implemented with the same bandwidth of $\lambda=.5 \mathrm{rad} / \mathrm{s}(.08 \mathrm{~Hz})$. This low bandwidth was chosen so that the effect of thruster dynamics was minimal. Yoerger [15] gives a thorough discussion of the thruster dynamics problem and some control solutions. Unfortunately, the ABE will not have feedback of the thruster states. Therefore, the thruster dynamics act as an unknown disturbance.

In the following pages there are figures which give the response for each trajectory and each controller. The vehicle motion is shown alongside the desired trajectories in dashed lines. The actual thrusts are shown and denoted by $T H(i)$ where the index $i$ indicates the particular thruster. The tracking error and power consumption are also shown.

The control schemes calculate only the required total thrust in each direction. How that thrust is divided between the five thrusters depends on the trajectory. For track $I$, the forward thrust is divided so that a pitching moment is not given to the vehicle. This means that the lower forward thruster is commanded to give more than the upper thrusters. Pitching may perhaps be induced anyway if the lower thruster saturates.

For track II and track III, the thrust is divided equally among the three forward thrusters. For all trajectories, the vertical thrust is shared equally between the two 
vertical thrusters.

Figure 6-1 shows the PD controller for the first trajectory. The position is nicely controlled with tracking convergence in 12 seconds. There is a significant overshoot of 20.5 percent in the velocity response with an oscillation with a 12 second period. This is the period which corresponds to a bandwidth of $.5 \mathrm{rad} / \mathrm{s}$.

The thrust plot shows how most of the forward motion is driven by the lower thruster. There is a period of saturation which is due to the short desired ramp time. This ramp time should not pose a problem for the actual vehicle since the power requirement is only 40 watts. From figure 6-2, the net power consumed after one minute is 0.2 watt-hours.

Figure 6-3 shows how the sliding mode controller does on the same trajectory. The first notable difference is that the sliding controller tracks much more precisely and converges more quickly. The overshoot on the velocity tracking is 2 percent compared to 20 percent for the PD control. The maximum tracking error is an order of magnitude less than that for PD control.

The thrust plot shows that this performance has its price. There is much more control activity than in the PD case. There is also a significant vertical thrust that is practically nonexistent in the PD case. The surprising result is that this control activity does not increase the net input power significantly. Figure 6-4 shows that the power consumed is 0.21 watt-hours which is an increase

The much improved performance of the sliding mode controller suggests that the comparison between the two controllers may not be fair. One measure of the fairness of the comparison is whether or not the bandwidths of the two controllers are the same. For the sliding mode controller, the "bandwidth" can be regarded as the parameter $\lambda$ which governs the speed of tracking convergence. The sliding mode control velocity tracking has an observed period around 5 seconds. This would correspond to a bandwidth of $1.25 \mathrm{rad} / \mathrm{s}$. A simple test was done to make certain that the "bandwidth" of the sliding mode controller is the same as that for the PD controller.

Track I was repeated with no thruster dynamics and a step disturbance in the 

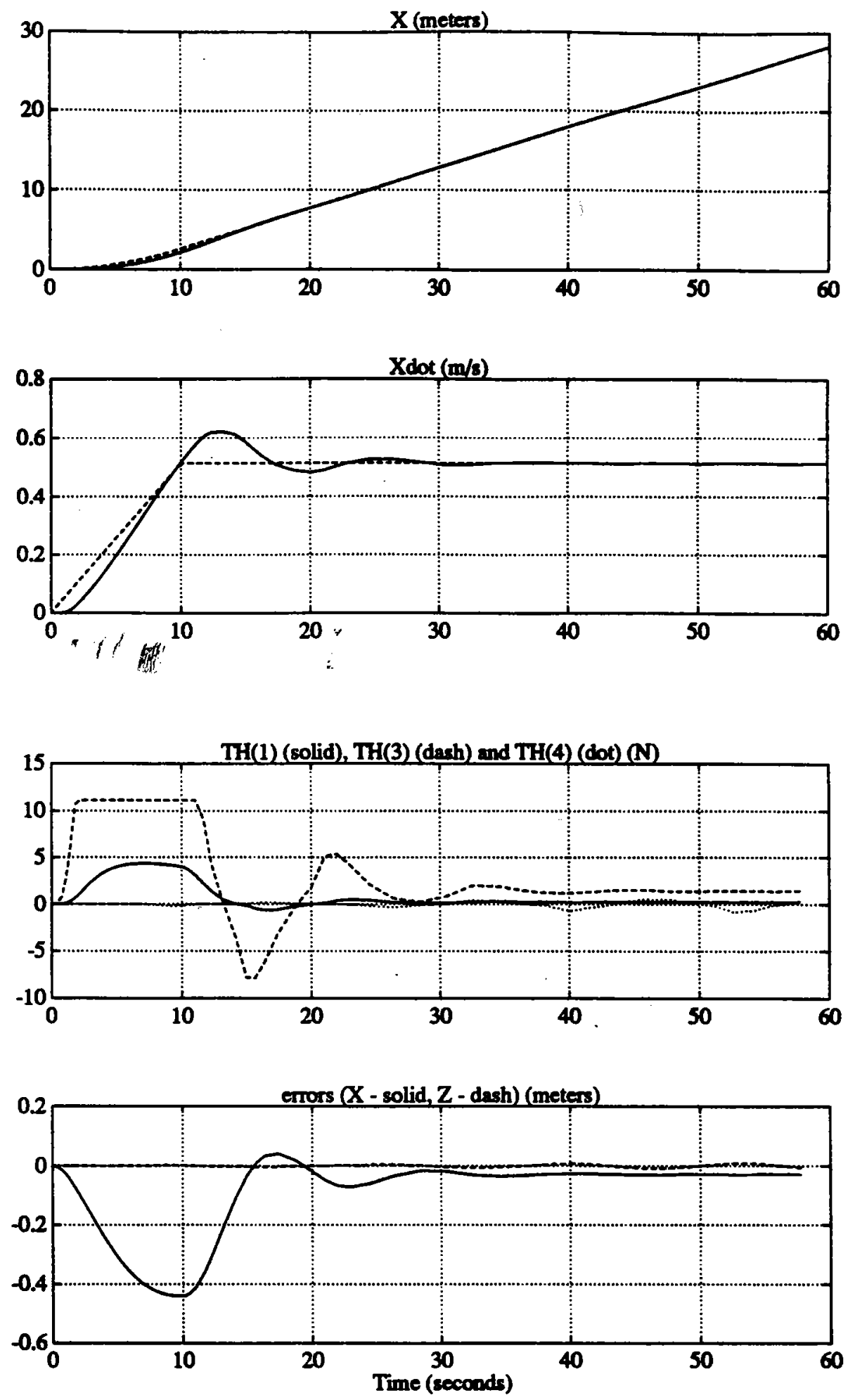

Figure 6-1: PD Control Performance on Track I 

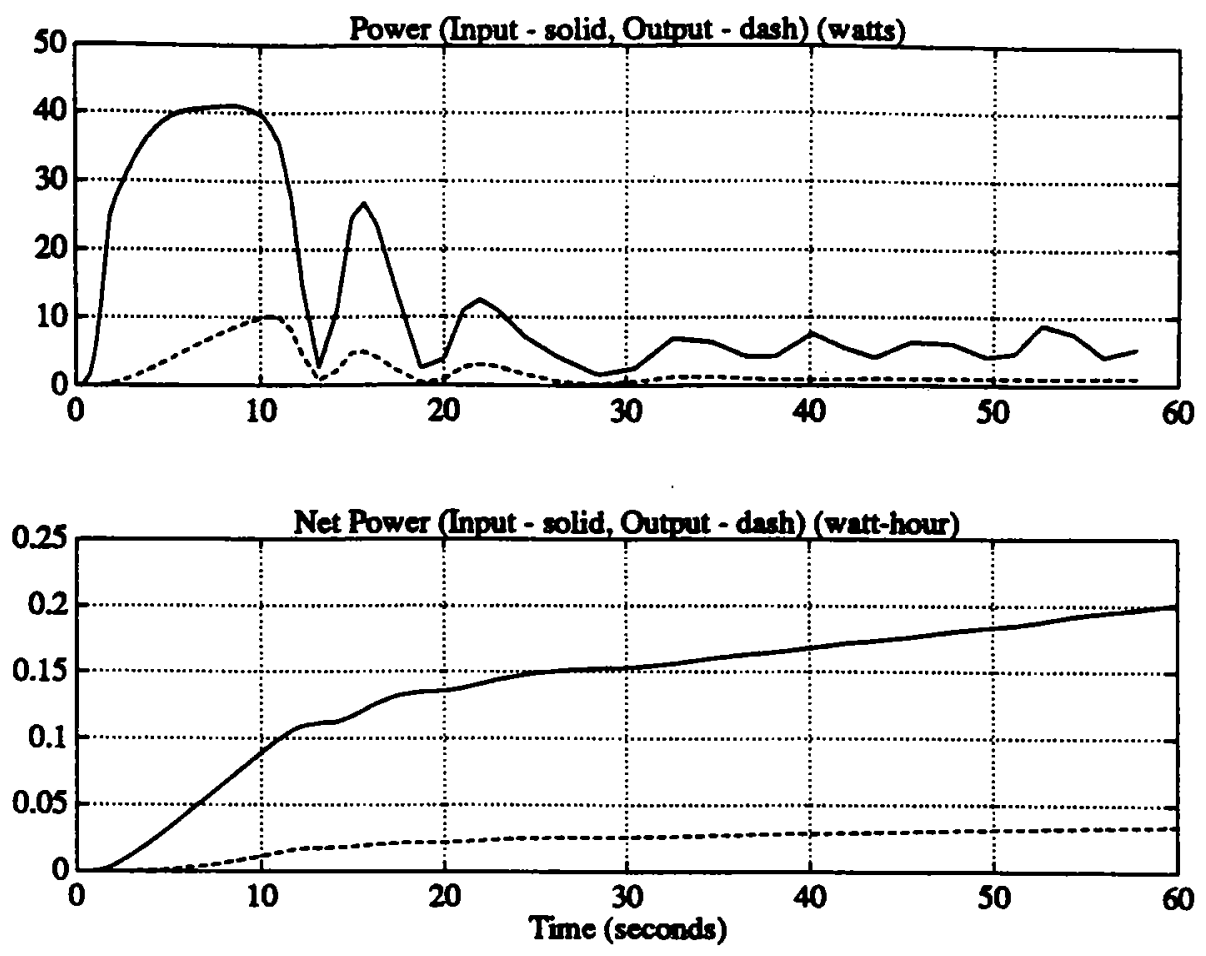

Figure 6-2: PD Control Power Consumption on Track I

desired velocity. Figure 6-5 shows the result. First, the 5 second oscillation is nonexistent which proves that is was associated with the thruster dynamics. The second observation is that the response to a step in desired velocity takes 12 seconds to recover good tracking. This is positive proof that the sliding mode controller bandwidth is $.5 \mathrm{rad} / \mathrm{s}$ and that the comparison is legitimate.

It is important to note that the power consumption results are a great improvement on the hand calculation. The steady state power computed by hand is given by

$$
\text { Power }=\text { Drag } \cdot \text { Velocity } \cdot \text { Ef ficiency }
$$

where the efficiency is around 0.6 for a typical thruster. For a cruise speed of 1 knot, this results in a power requirement of 1.6 watts. Figure $6-2$ indicates that the cruising power requirement is around 6 watts. The hand calculation is off by more than a factor of three. This is a good example of the usefulness of these simulations. 

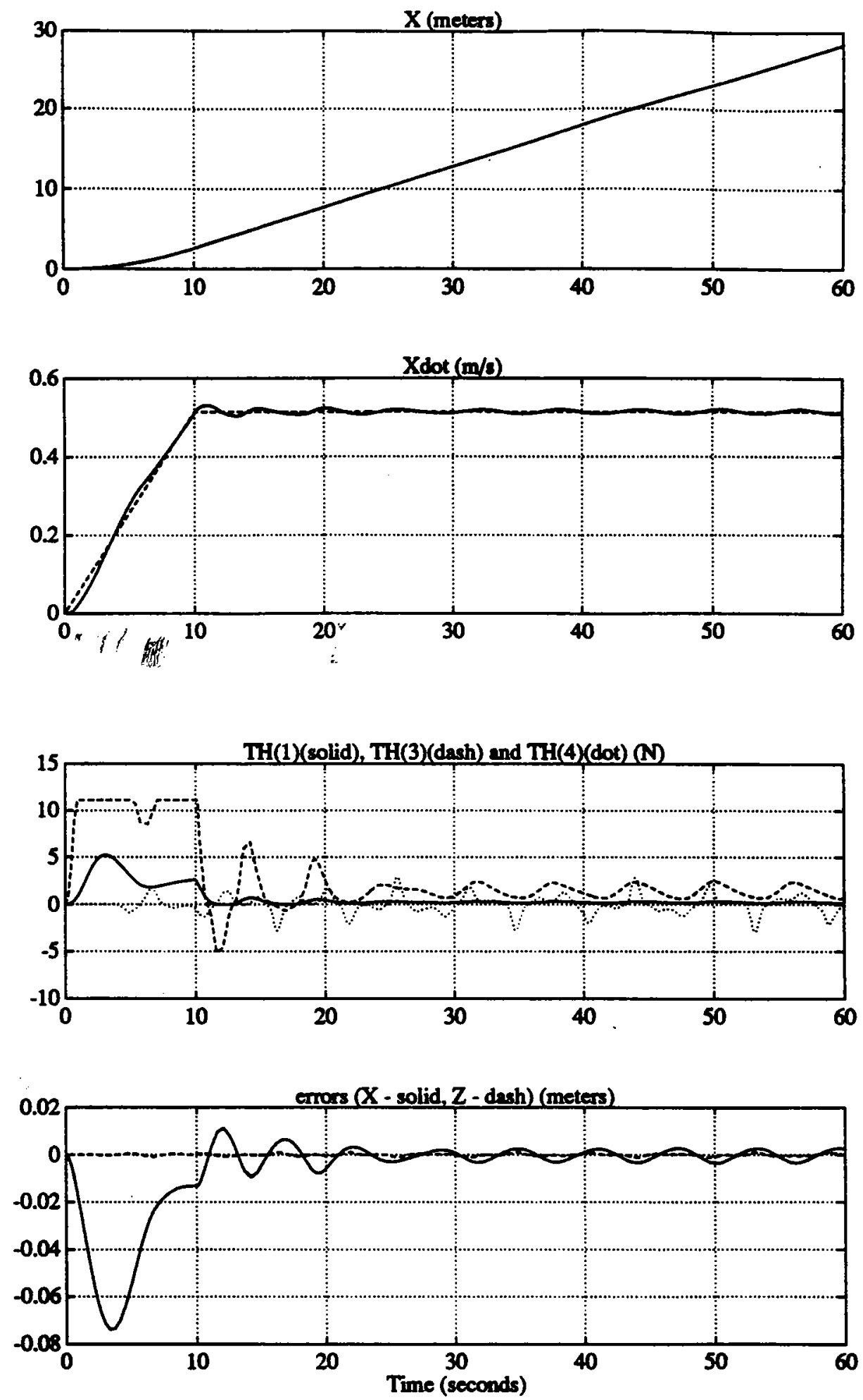

Figure 6-3: Sliding Mode Control Performance on Track I 

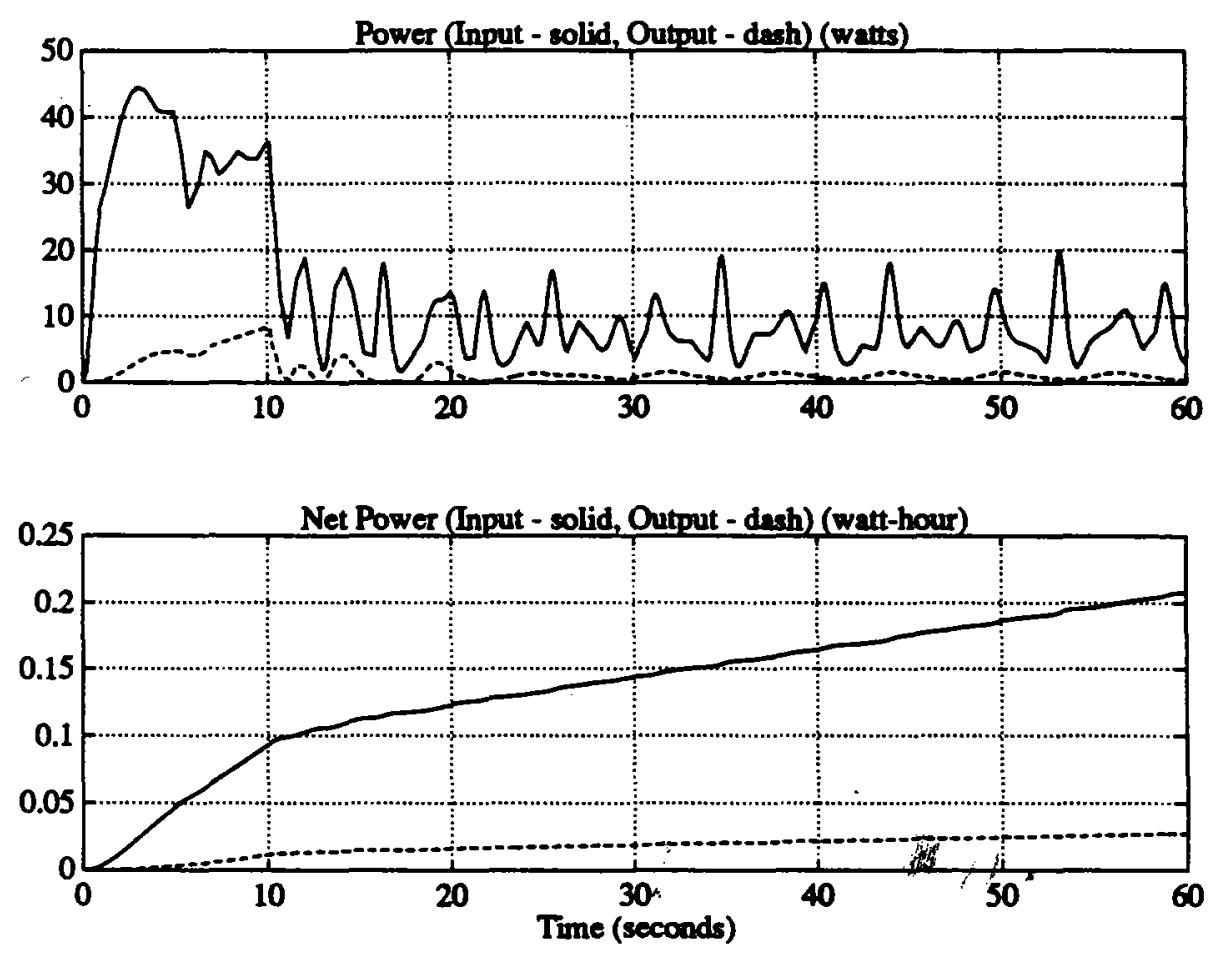

Figure 6-4: Sliding Mode Control Power Consumption on Track I 

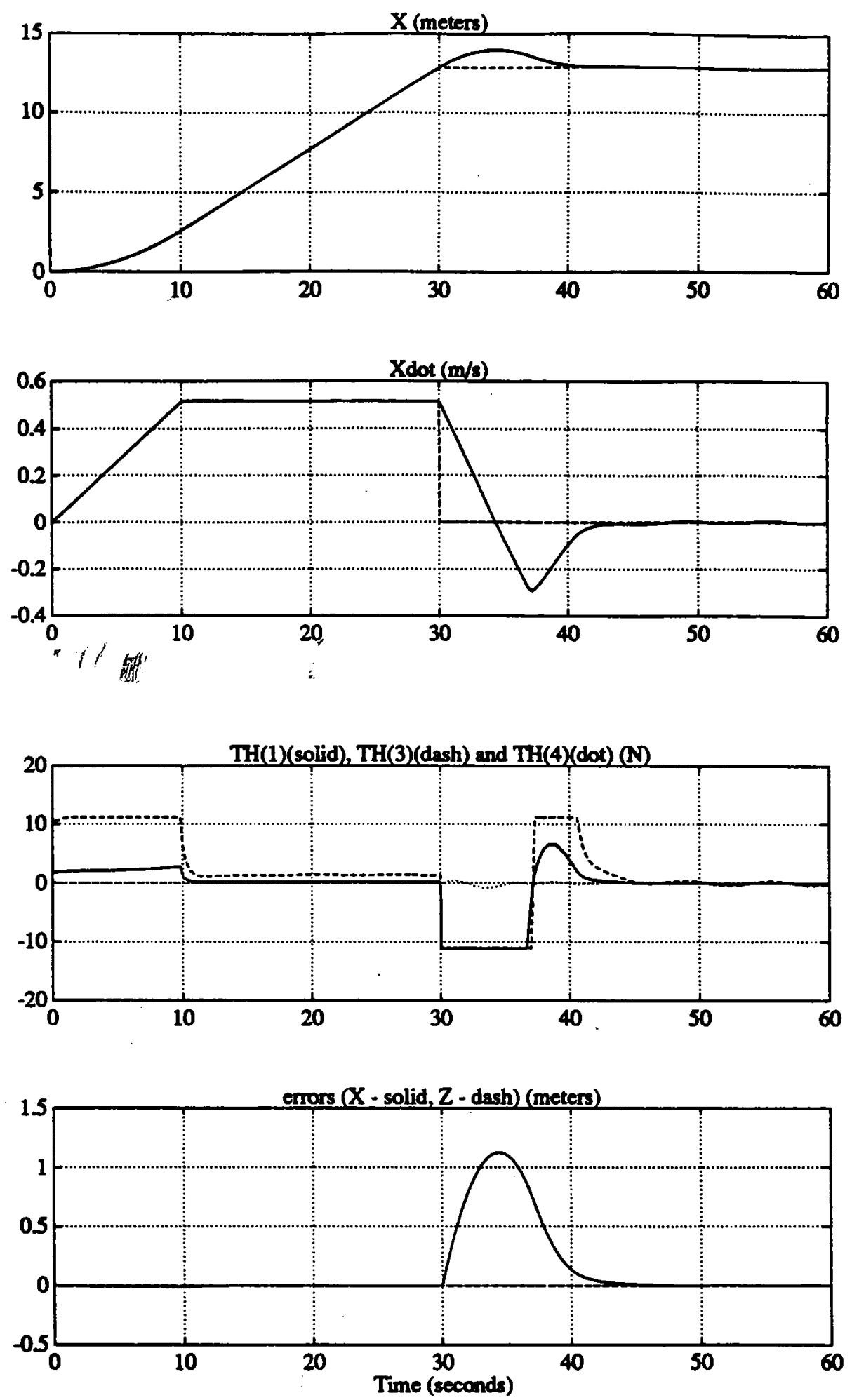

Figure 6-5: Bandwidth Test for Sliding Mode Control on Track I 
Figure 6-6 shows the response for the PD controller on track II. The behavior is similar to that for track I. There is a slight improvement in tracling the vertical direction because there is more drag. The added damping in the vertical direction naturally supplies derivative gain and improves the performance. Figure 6-7 shows the power consumed in 60 seconds is 0.25 watt-hours.

The sliding mode control performance for track II is shown in figure 6-8. There is a similar improvement in tracking the vertical direction. Again, the sliding mode control tracks much tighter than the PD controller. Figure 6-9 shows that the power consumed is the same. 

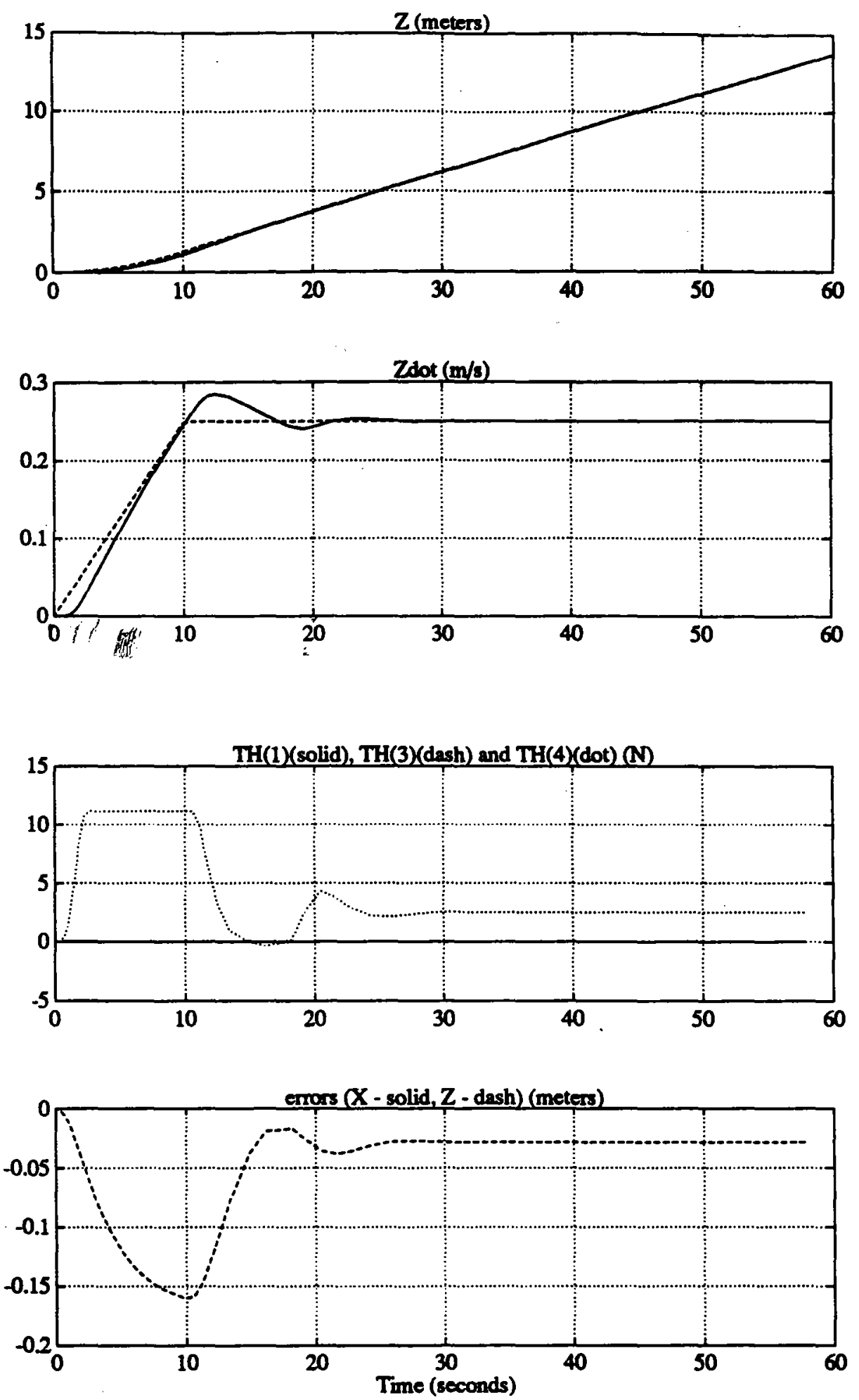

Figure 6-6: PD Control Performance on Track II 

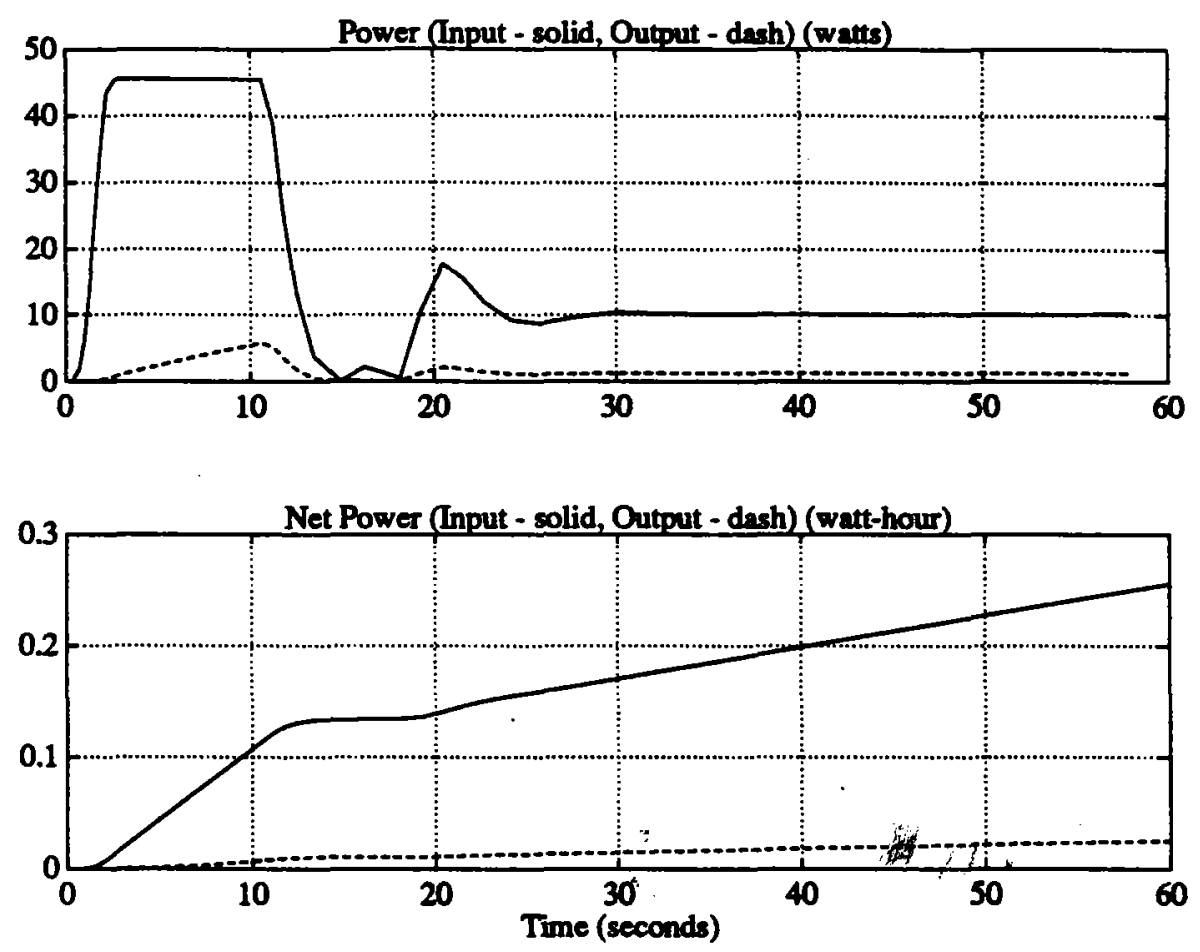

Figure 6-7: PD Control Power Consumption on Track II 

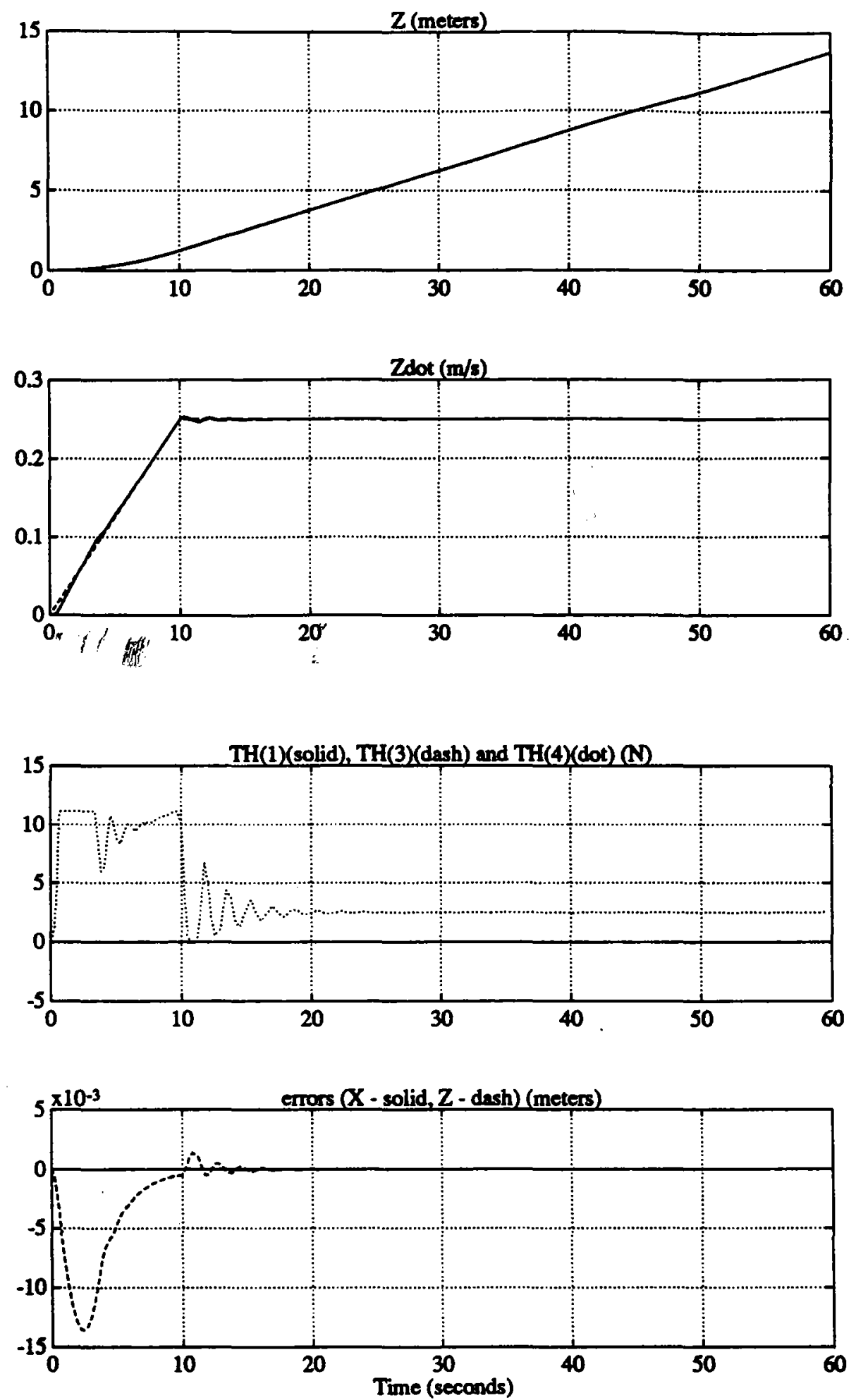

Figure 6-8: Sliding Mode Control Performance on Track II 

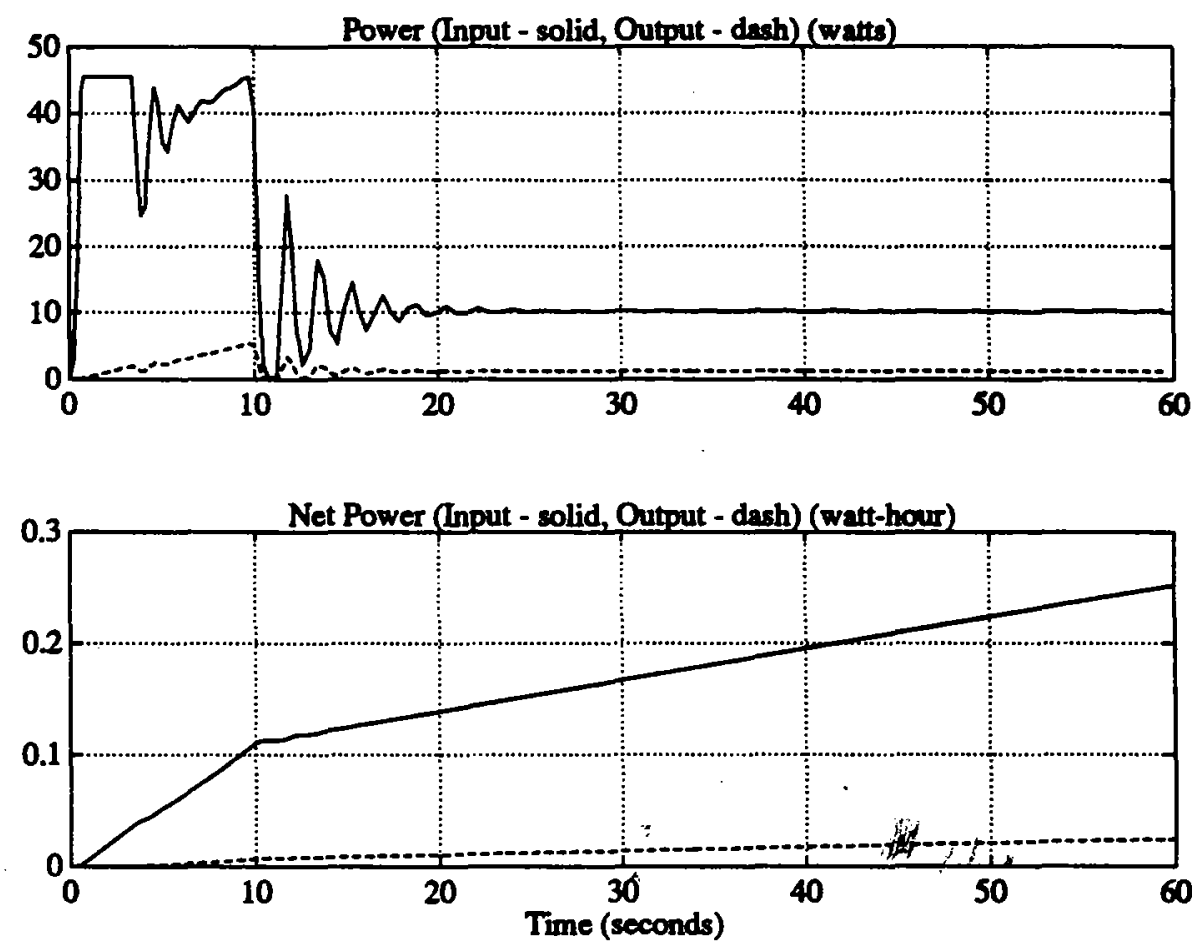

Figure 6-9: Sliding Mode Control Power Consumption on Track II 
The PD control performance on the combined forward/vertical maneuver is shown in figure 6-10. There is good tracking for both $X$ and $Z$ position with some overshoot in the velocity responses. As noted before, the tracking performance is much better in the $Z$ direction. This trajectory is unsophisticated in that it does not reflect any knowledge about the vehicle dynamics. It is a brute force way of getting from point A to point B. A more intelligent approach will be presented in the next section.

Figure 6-12 shows the track III vehicle response under sliding mode control. The results are as expected. There is much improvement in the tracking performance over the PD controller. Comparison of figures 6-11 and 6-13 show that the extra power consumed by the sliding mode controller is only 0.03 watt-hours.

Table 6.1 is a summary of the performance measures for the two controllers. Sliding mode control is is bensistently better than PD control with the same bandwidth. This is not surprising since with sliding control there is a mechanism for adjusting the gains dynamically to best utilize the available bandwidth. When the uncertainty is large, the gains are increased to counter it. The gain selection depends on the current state of the system whereas PD control is required to have constant gain.

Table 6.2 is a summary of the power requirement for cruising on each trajectory. The average power is computed by dividing the net power gain by a time for which there is no acceleration. The powers given are for PD control.

Table 6.1: Controller Performance Comparison

\begin{tabular}{|c|c|c|c|c|}
\hline Track & Controller & $\begin{array}{c}\text { Overshoot } \\
\%\end{array}$ & $\begin{array}{c}\text { Maximum } \\
\text { Error (m) }\end{array}$ & $\begin{array}{c}\text { Power } \\
\text { (watt-hours) }\end{array}$ \\
\hline \hline I & PD & 20.5 & 0.45 & 0.20 \\
& SMC & 2 & 0.07 & 0.21 \\
\hline II & PD & 12 & 0.16 & 0.25 \\
& SMC & 0 & 0.01 & 0.25 \\
\hline III & PD & 15 & 0.18 & 0.54 \\
& SMC & 6 & 0.04 & 0.57 \\
\hline
\end{tabular}



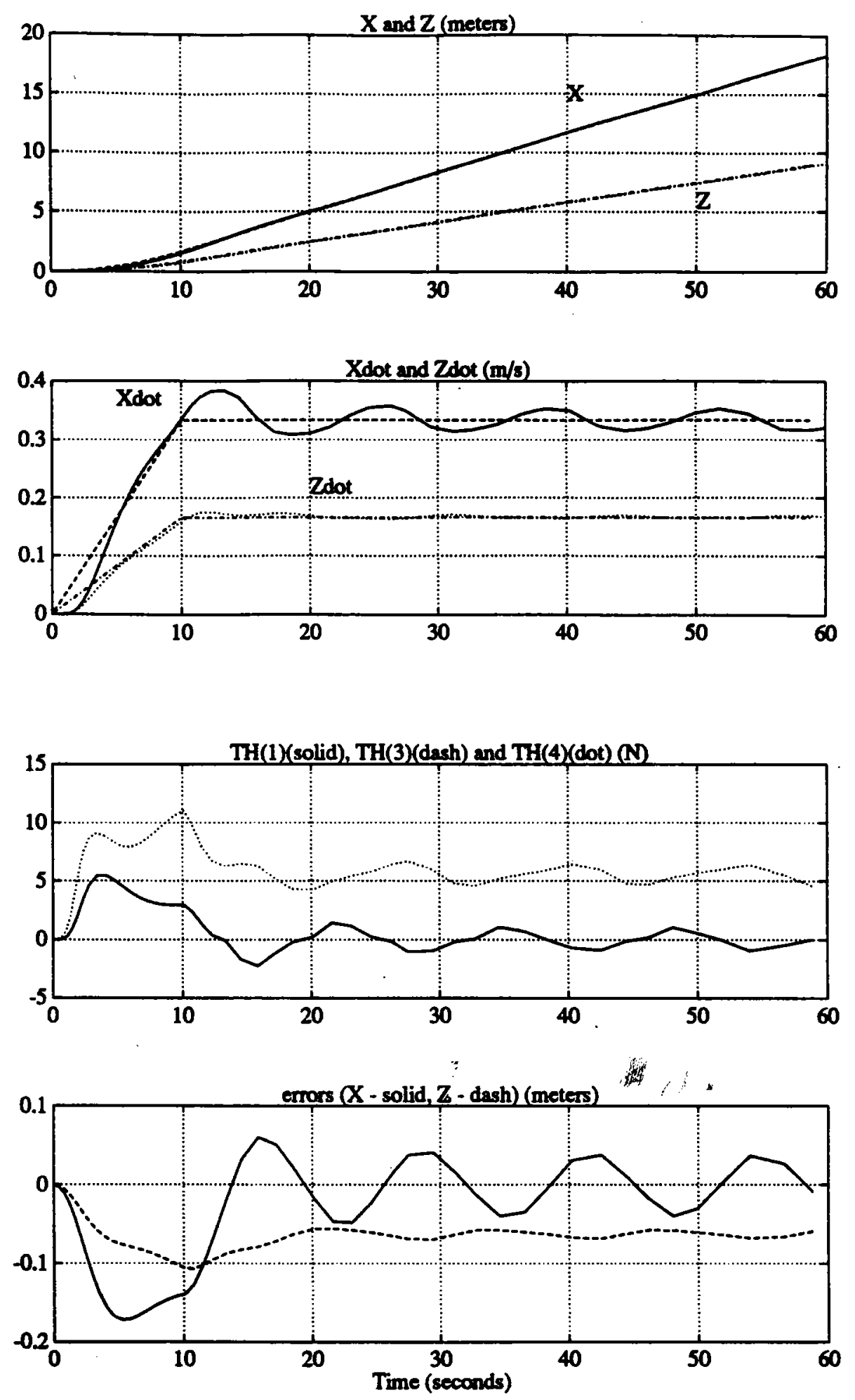

Figure 6-10: PD Control Performance on Track III 

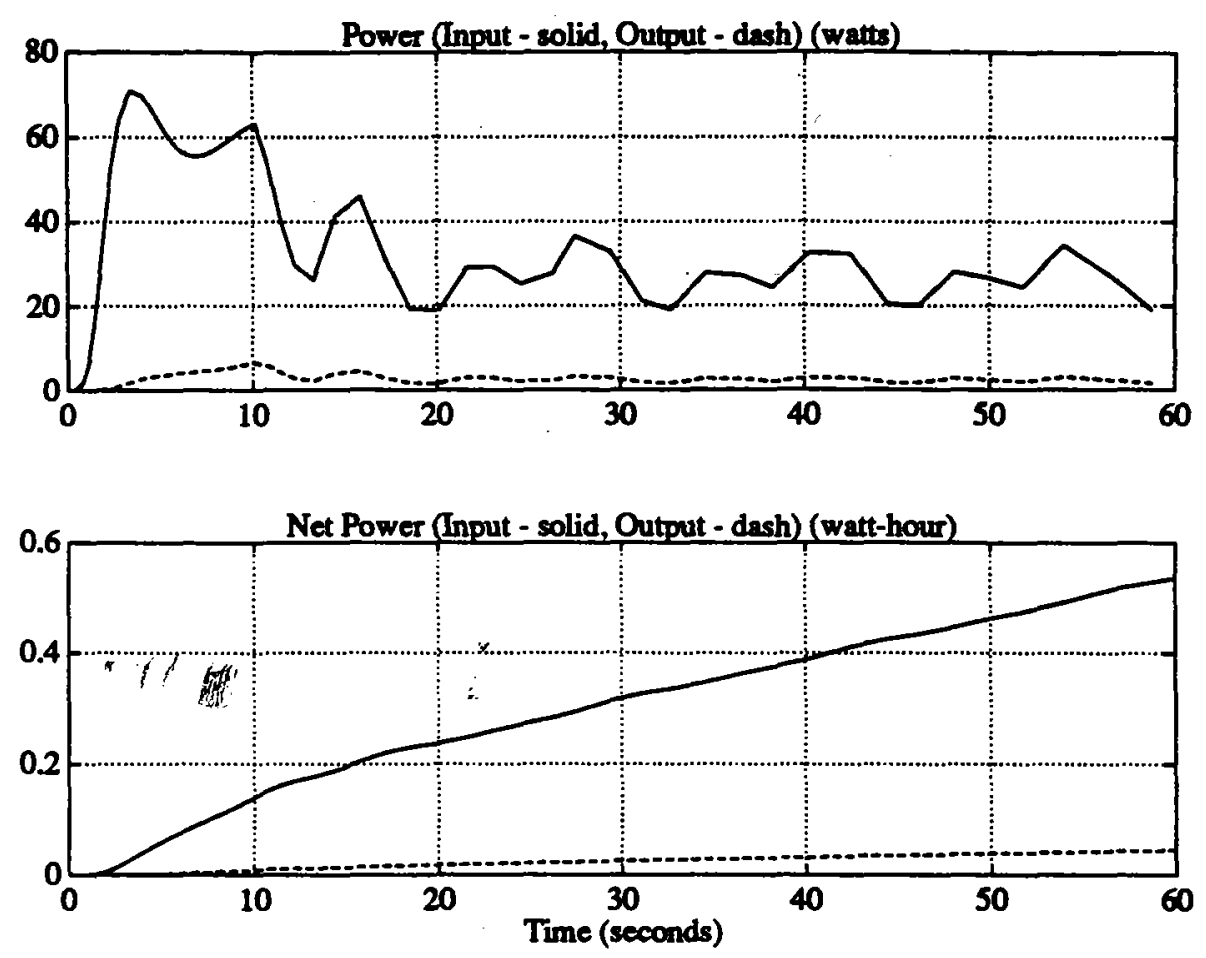

Figure 6-11: PD Control Power Consumption on Track III

Table 6.2: Average Power Requirement for Cruising

\begin{tabular}{|c|c|}
\hline Track & Power (watts) \\
\hline \hline I & 6.0 \\
\hline II & 9.6 \\
\hline III & 28.8 \\
\hline
\end{tabular}



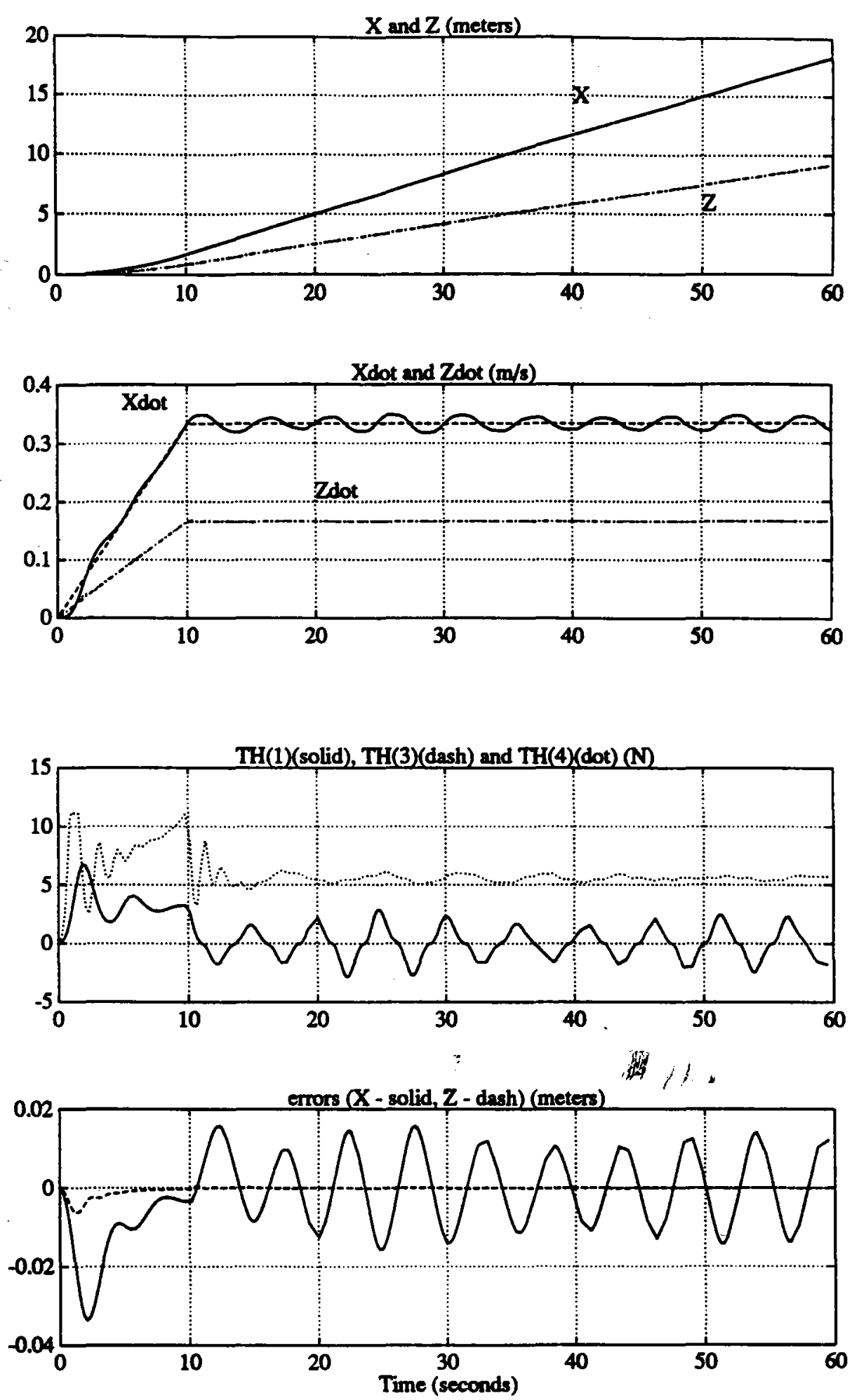

Figure 6-12: Sliding Mode Control Performance on Track III 

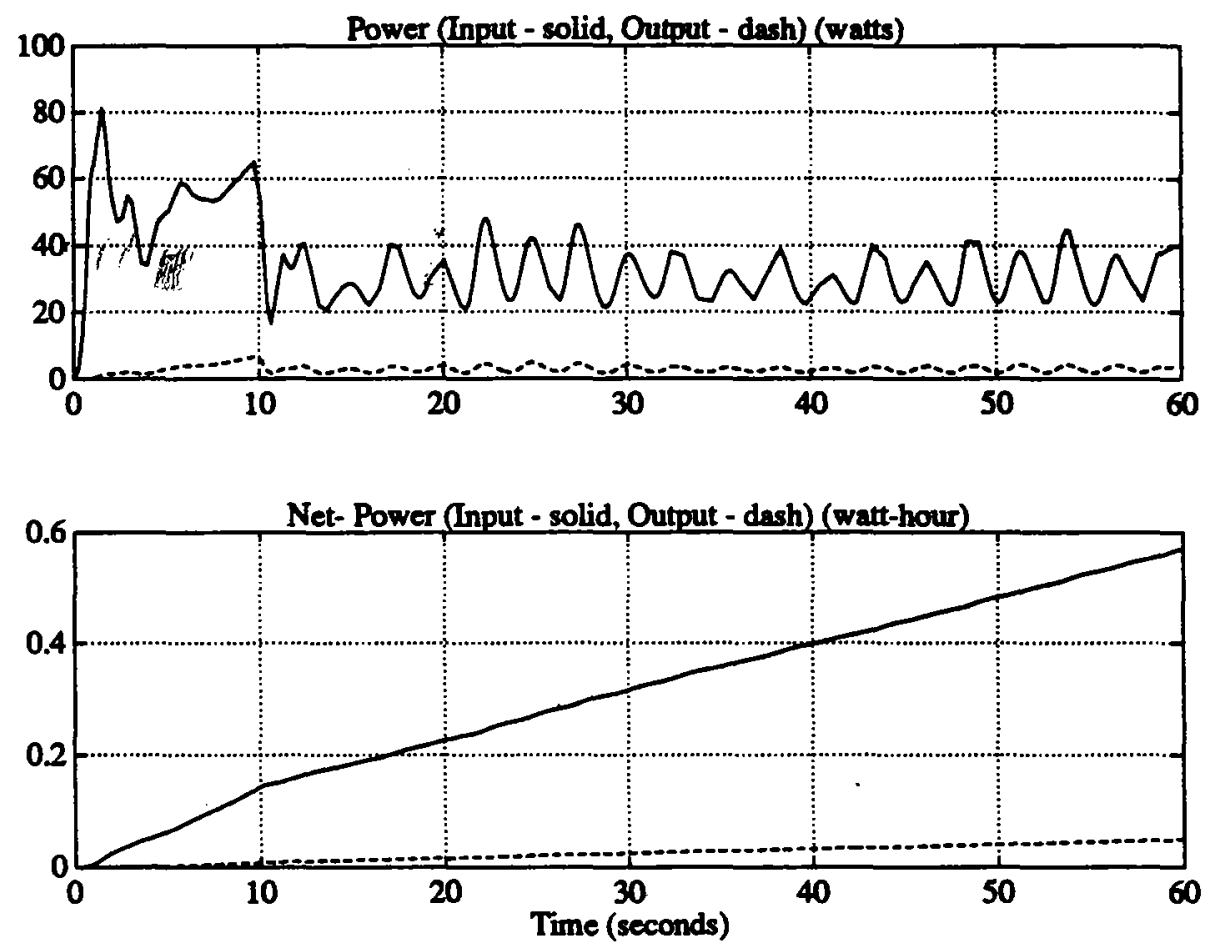

Figure 6-13: Sliding Mode Control Power Consumption on Track III 


\subsection{Combined Forward/Vertical Motion Revisited}

It was mentioned previously that the third trajectory was a brute force way of moving forward and down at the same time. A more intuitive method is to pitch the vehicle so that there is no angle of attack. The forward direction has the minimum drag so it makes sense to always move the vehicle forward instead of a combined motion which gives large drag forces.

The ABE will have a movable weight with which it can quasistatically adjust its pitch angle. For a coordinated $X-Z$ maneuver, the pitch can be adjusted to match the desired glide angle. Then, if the forward thrust is divided so that no pitching is induced, the power required to stay on trajectory should be smaller than for the brute force case.

This hypothesis was tested on a trajectory similar to track III. Appropriate initial conditions were given to the vehicle to make it line up with the trajectory. Figure 6-14 shows the vehicle trajectory and thrusts for PD control. The short line segments on the $X-Z$ plot indicate the pitch angle of the vehicle. The power consumed in 60 seconds is 0.18 watt-hours.

Figure 6-15 shows the response for the dumb power down method. The power consumed for this trajectory is 0.225 watt-hours and there is significant vertical control action. The new method has a power savings of 0.045 watt-hours. This is slightly more than what the vertical thrusters consume on the original trajectory ( 0.04 watthours). 18 percent of the power is saved by simply pitching the vehicle to match the glide angle.

In this chapter, the simulation model and controllers were put through some anticipated ABE maneuvers. The sliding mode control methodology consistently provided better tracking performance than a PD controller with the same bandwidth and input power. Insight was gained by simulating different methods for combined forward/vertical motion. For all trajectories, the simulations gave realistic estimates of the power required for tracking control. 

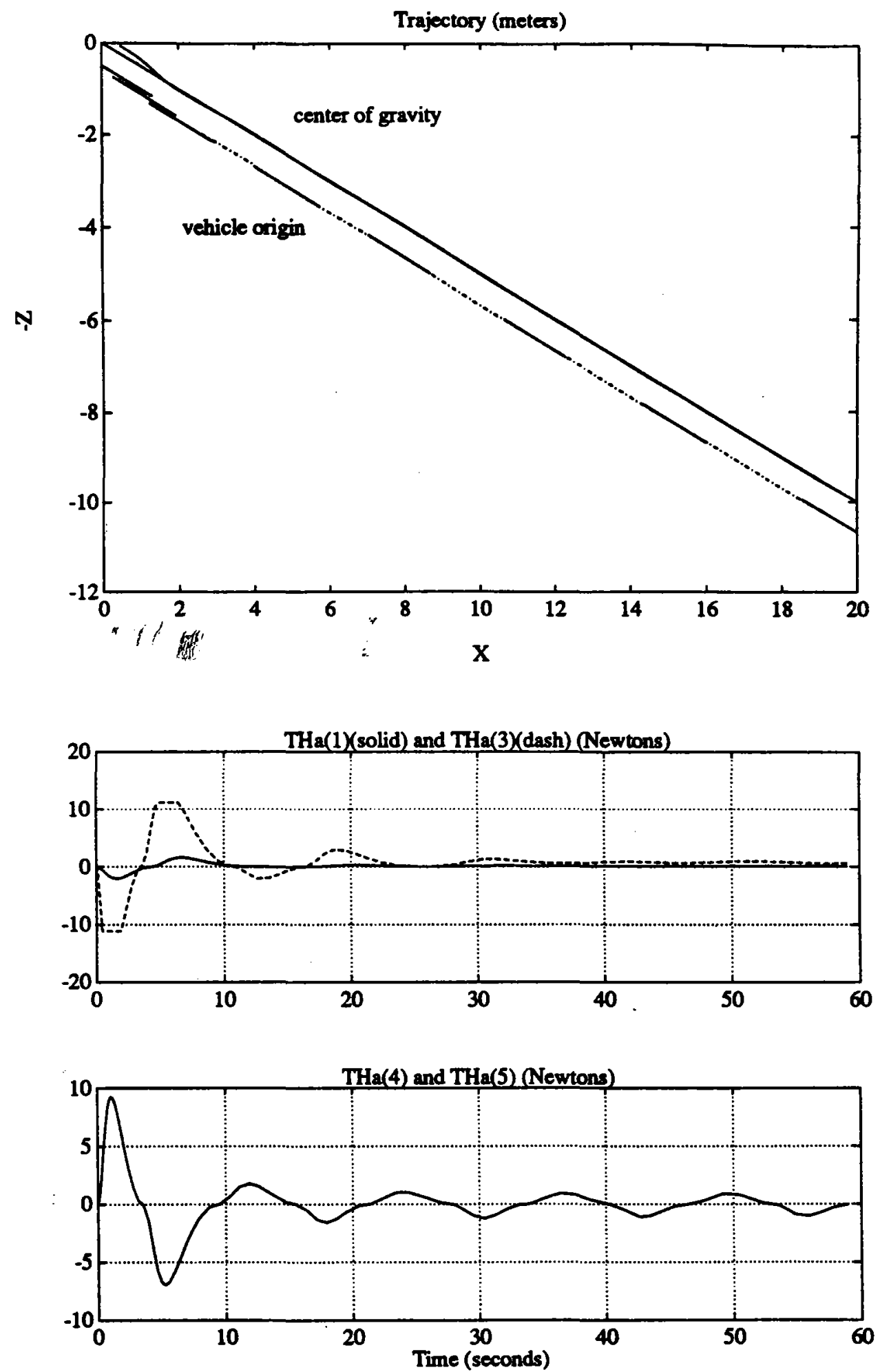

Figure 6-14: Forward/Vertical Maneuver with Pitch Angle Control 

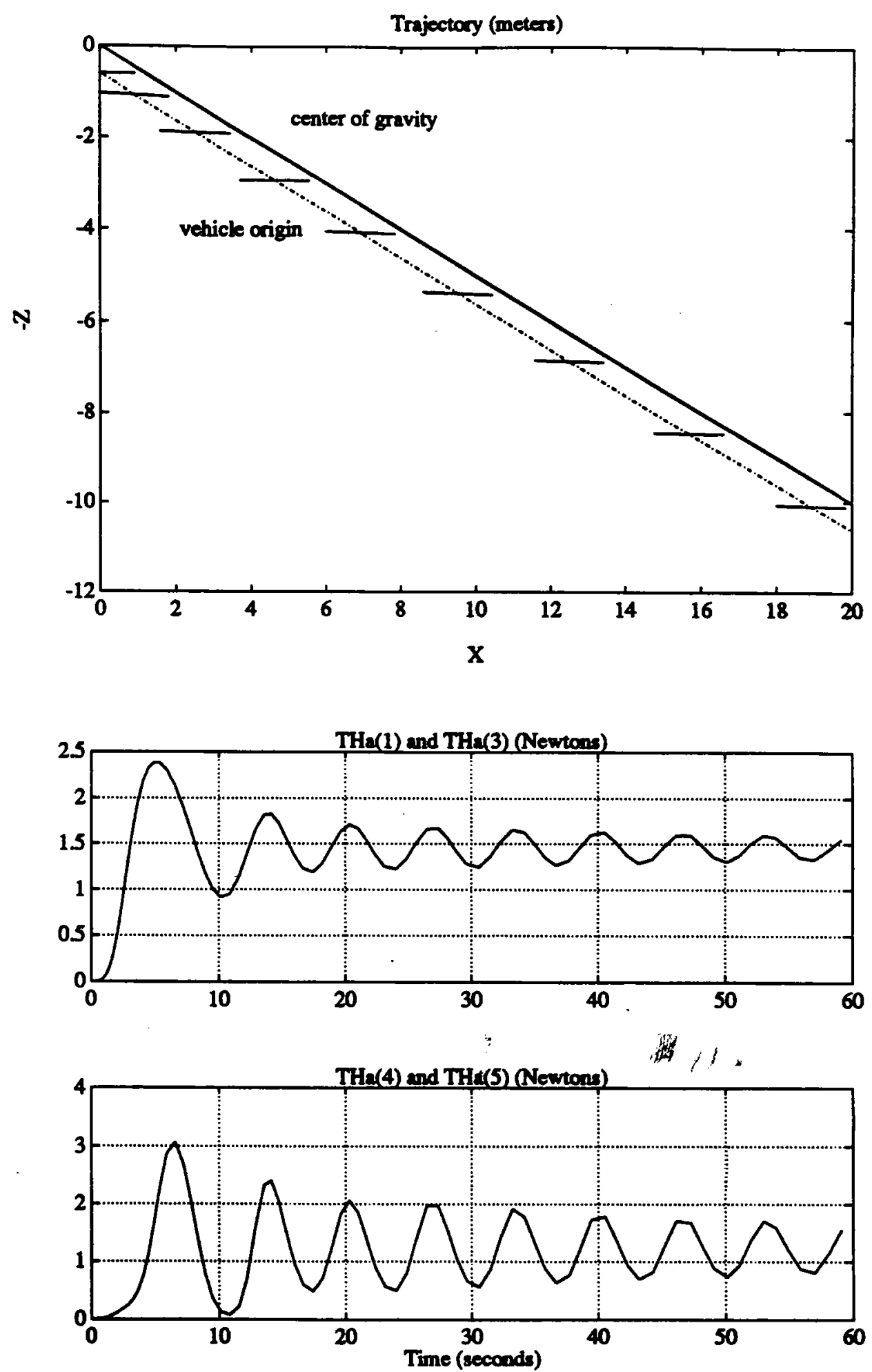

Figure 6-15: Forward/Vertical Maneuver without Pitch Control 


\section{Chapter 7}

\section{Conclusions and}

\section{Recommendations for Future}

\section{Work "}

The goal of this thesis was to develop a good understanding of the ABE longitudinal dynamics. These motions are important in ABE trajectories which involve forward or vertical motion and combinations of the two. A model was developed specifically for the ABE which included experimental drag measurements and a verified thruster model. This model was then examined in computer simulation. Two different control methodologies were used for trajectory control. The simulations provided valuable information on how the ABE will perform and how much power it will consume.

The scale model experiment was discussed in chapter 2 . The principal drag coefficients were measured and found to be quite low due to the streamlined nature of the vehicle. Some interesting drag/lift relationships were observed when the pitch angle of the vehicle was varied. The drag force variation was similar to that of a bluff body whereas the lift force acted as if it was a slender body. A region of stall between 5 and 20 degrees was observed for which there is no lift increase for increased angle of attack. The measured lift/drag data are very important since they completely capture the bluff and slender natures of the ABE geometry.

The experimental data were incorporated into a model of the ABE dynamics in 
chapter 3. Traditional hydrodynamic modelling techniques were used to formulate a model for the longitudinal plane. A detailed discussion was given about the forces on the vehicle and the predicted hydrodynamics coefficients. An experimentally verified model of the thruster dynamics was also included.

A computer simulation was used to verify the model and understand the basic characteristics of the ABE geometry. Some basic behaviors and simulation limitations were presented in chapter 4. A pendulum mode with a period of 6.4 seconds was found to dominate the vehicle response. The drag force dependence on angle of attack was demonstrated to be very important in how fast drag will stop the vehicle.

Two controllers were developed in chapter 5 to predict the power requirements for trajectory control. A PD controller with nonlinear feedforward and a sliding mode controller were designed with the same bandwidth. Neither controller contained prior knowledge about the thruster dynamics. The slow thruster response was considered to be a disturbance on the system.

Both controllers were tested on three simple ABE trajectories. The power requirement for each controller was calculated and found to be nearly the same. However, sliding mode control provided much tighter tracking than PD control. The sliding mode control performs better because the gains are dynamically adjusted to best use the available controller bandwidth.

An intuitive way to maneuver the vehicle forward and was found to save substantial power. A power savings of 18 percent was observed by simply altering the pitch angle of the vehicle to match the desired glide angle. This was a good example of how the developed simulation is useful. Trajectories and control strategies of interest can be simulated to show the benefits or costs.

There is much room for future work on this project. The ABE design is continually evolving and the model must be updated to match the design changes. More scale model tests should be done to obtain better estimates of the pitch damping coefficient and added inertia in pitch. When the full scale vehicle is available, system identification techniques should be used to fine tune the model. 


\section{Appendix A}

\section{Refining the Equations of Motion}

This section gives the detailed development of the two input/two output equations of motion for the X- $\mathrm{X}$ plane. The equations of motion as developed in chapter 3 can be compactly written as

$$
\left[\begin{array}{c}
\dot{u} \\
\dot{w} \\
\dot{q}
\end{array}\right]=I^{-1}\left[\begin{array}{c}
\Sigma F_{z}+X_{\text {thruet }} \\
\Sigma F_{z}+Z_{\text {thruet }} \\
\Sigma M+M_{\text {thruet }}
\end{array}\right]
$$

$I$ is the inertia matrix. The sum terms on the right hand side incorporate all of the remaining dynamics and forces on the vehicle excluding the thrust inputs. $X_{\text {thruot }}$ is the net thrust from thrusters $T_{1}, T_{2}$ and $T_{3}$. Similarly, $Z_{\text {thruet }}$ is the thrust from $T_{4}$ and $T_{5}$ combined. The input thrust moment can be written in terms of these two thrusts

$$
M_{\text {thruat }}=X_{\text {thruet }} \text { arm } x+Z_{\text {thrust }} \text { arm }
$$

where the generalized moment arms are

$$
a r m_{=}=\frac{T_{2}\left(z_{T 2}-z_{g}\right)+T_{3}\left(z_{T 3}-z_{g}\right)+T_{1}\left(z_{T 1}-z_{g}\right)}{T_{1}+T_{2}+T_{3}}
$$




$$
\operatorname{arm}_{2}=\frac{-T_{4}\left(x_{T_{4}}-x_{g}\right)-T_{5}\left(x_{T 5}-x_{g}\right)}{T_{4}+T_{5}}
$$

Since we are concerned with control of the inertial quantities $X_{0}$ and $Z_{0}$, the equations of motion must be written in terms of these parameters. From the development of the equations of motion, it is known that

$$
\begin{gathered}
\dot{X}_{0}=u \cos \theta+w \sin \theta \\
\dot{Z}_{0}=-u \sin \theta+w \cos \theta \\
\dot{\theta}=q
\end{gathered}
$$

If these equations are differentiated with respect to time the result is

$$
\begin{gathered}
\ddot{X}_{0}=\dot{u} \cos \theta-u \dot{\theta} \sin \theta+\dot{w} \sin \theta+w \dot{\theta} \cos \theta \\
\dot{Z}_{0}=-\dot{u} \sin \theta-u \dot{\theta} \cos \theta+\dot{w} \cos \theta-w \dot{\theta} \sin \theta \\
\dot{\theta}=\dot{q}
\end{gathered}
$$

The general equation of motion above can be substituted in directly where the time derivatives of the state appear. Since $\theta$ will not be actively controlled, the condensed dynamics are two-dimensional and can be written in the following format

$$
\left[\begin{array}{c}
\tilde{X}_{0} \\
\tilde{Z}_{0}
\end{array}\right]=\vec{f}(u, w, q, \theta)+B\left[\begin{array}{l}
X_{\text {thruat }} \\
Z_{\text {thruot }}
\end{array}\right]
$$

$\vec{f}$ is a vector function of the state with the following elements

$$
\begin{aligned}
f(1)=\left(I^{-1}(1,1) \Sigma F_{z}+I^{-1}(1,2) \Sigma F_{z}\right. & \left.+I^{-1}(1,3) \Sigma M+w q\right) \cos \theta+\left(I^{-1}(2,1) \Sigma F_{z}\right. \\
& \left.+I^{-1}(2,2) \Sigma F_{z}+I^{-1}(2,3) \Sigma M-u q\right) \sin \theta
\end{aligned}
$$




$$
\begin{array}{r}
f(2)=-\left(I^{-1}(1,1) \Sigma F_{z}+I^{-1}(1,2) \Sigma F_{z}+\right. \\
\left.I^{-1}(1,3) \Sigma M+w q\right) \sin \theta+\left(I^{-1}(2,1) \Sigma F_{z}\right. \\
\left.+I^{-1}(2,2) \Sigma F_{z}+I^{-1}(2,3) \Sigma M-u q\right) \cos \theta
\end{array}
$$

$B$ is a $2 \times 2$ matrix with the following elements

$$
\begin{gathered}
B(1,1)=\left(I^{-1}(1,1)+I^{-1}(1,3) a r m_{z}\right) \cos \theta+\left(I^{-1}(2,1)+I^{-1}(2,3) a r m_{z}\right) \sin \theta \\
B(1,2)=\left(I^{-1}(1,2)+I^{-1}(1,3) a r m_{z}\right) \cos \theta+\left(I^{-1}(2,2)+I^{-1}(2,3) a r m_{z}\right) \sin \theta \\
B(2,1)=-\left(I^{-1}(1,1)+I^{-1}(1,3) a r m_{z}\right) \sin \theta+\left(I^{-1}(2,1)+I^{-1}(2,3) a r m_{z}\right) \cos \theta \\
B(2,2)=-\left(I^{-1}(1,2)+I^{-1}(1,3) a r m_{z}\right) \sin \theta+\left(I^{-1}(2,2)+I^{-1}(2,3) a r m_{z}\right) \cos \theta
\end{gathered}
$$

There is a simple transformation to convert the dynamics describing $X_{0}$ and $Z_{0}$ to equations in $X_{e g}$ and $Z_{e g}$. From simple geometry, we have

$$
\begin{aligned}
& X_{c g}=X_{0}+z_{g} \sin \theta \\
& Z_{c g}=Z_{o}+z_{g} \cos \theta
\end{aligned}
$$

If these relations are differentiated twice, the accelerations are

$$
\begin{aligned}
& \bar{X}_{c g}=\bar{X}_{o}-z_{g} q^{2} \sin \theta \\
& \ddot{Z}_{c g}=\ddot{Z}_{o}-z_{g} q^{2} \cos \theta
\end{aligned}
$$

Thus, the dynamics for the center of gravity can be obtained by simply adding one term to each element of $\vec{f}$. 


\section{Bibliography}

[1] Anderson, J.M., Autonomous Benthic Explorer Scale Model Data, Woods Hole Oceanographic Institution Internal Memo, (1992).

[2] Abkowitz, M.A., Stability and Motion Control of Ocean Vehicles, The MIT Press, Cambridge, MA, (1969).

[3] Cooke V, J.G., "Incorporating Thruster Dynamics in the Control of an Underwater Vehicle", MIT/WHOI Ocean Engineers Thesis and MIT Masters Thesis, (1989).

[4] Crane, J.W. and Humphreys D.E., "Modelling and Simulation of Underwater Vehicles", SCS Summer Simulation Conference Professional Development Seminar Notes, (1991).

[5] Feldman, J., "DTNSRDC Revised Standard Submarine Equations of Motion", DTNSRDC/SPD-0393-09, (1979).

[6] Hoerner, S.F., Fluid-Dynamic Drag, Hoerner Fluid Dynamics, (1965).

[7] Hopkins, E.J., "A Semiempirical Method for Calculating the Pitching Moment of Bodies of Revolution at Low Mach Numbers", NACA RM A51C14, (1951).

[8] Humphreys, D.E. and Watkinson, K.W., "Prediction of Acceleration Hydrodynamic Coefficients for Underwater Vehicles from Geometric Parameters", NCSL TR 327-78, (1978).

[9] Lamb, H., Hydrodynamics, Dover, (1945). 
[10] Newman, J.N., Marine Hydrodynamics, The MIT Press, Cambridge, MA (1977).

[11] Slotine, J.-J.E. and Li, W., Applied Nonlinear Control, Prentice-Hall, (1991).

[12] Slotine, J.-J.E., "The Robust Control of Robot Manipulators", Int. J. Robotics Research, 4,2, (1985).

[13] Waller, E.V., "Design and Full-Scale Testing of a Sliding Mode Controller for a Small Underwater Vehiclen, MIT Masters Thesis, (1989).

[14] Woodford, T.J., "Propulsion Optimization for ABE, an Autonomous Underwater Vehicle (AUV)", MIT Masters Thesis, (1991).

[15] Yoerger, D.R. S. Sooke, J.G. and Slotine, J.-J.E., "The Influence of Thruster Dynamics on Underwater Vehicle Behavior and Their Incorporation Into Control System Design", IEEE J. of Oceanic Engineering, Vol. 15, No. 9, July, pp.167-178, (1990).

[16] Yoerger, D.R., Bradley, A.M. and Walden, B.B., "A Deep Ocean AUV for Scientific Seafloor Survey-The Autonomous Benthic Explorer", Unmanned Systems, Vol. 9, No. 2, Spring, pp.17-23, (1991). 